\title{
Rethinking the Fast-Slow Continuum of Individual Differences
}

\author{
Marco Del Giudice \\ University of New Mexico
}

In press: Evolution and Human Behavior (accepted manuscript)

Marco Del Giudice, Department of Psychology, University of New Mexico. Address correspondence to Marco Del Giudice, Department of Psychology, University of New Mexico. Logan Hall, 2001 Redondo Dr. NE, Albuquerque, NM 87131, USA; email: marcodg@unm.edu 


\begin{abstract}
The idea that individual differences in behavior and physiology can be partly understood by linking them to a fast-slow continuum of life history strategies has become popular in the evolutionary behavioral sciences. I refer to this approach as the "fast-slow paradigm" of individual differences. The paradigm has generated a substantial amount of research, but has also come increasingly under scrutiny for theoretical, empirical, and methodological reasons. I start by reviewing the basic empirical facts about the fast-slow continuum across species and the main theoretical accounts of its existence. I then discuss the move from the level of species and populations to that of individuals, and the theoretical and empirical complications that follow. I argue that the fast-slow continuum can be a productive heuristic for individual differences; however, the field needs to update its theoretical assumptions, rethink some methodological practices, and explore new approaches and ideas in light of the specific features of the human ecology.
\end{abstract}

Keywords: balancing selection; developmental plasticity; ecological gambit; fast-slow continuum; genetics; individual differences; life history theory; pace-of-life syndromes; personality; physiology. 


\section{Introduction}

In this paper, I critically examine the idea that individual differences in behavior and physiology can be partly understood by linking them to a fast-slow continuum of life history strategies. In its original form, the fast-slow continuum denotes an empirical pattern of species differences in fitness-related traits such as fertility, mortality, and offspring size (Jeschke et al., 2008). Although there is still no complete, widely accepted theory of the fast-slow continuum, it is clear that some important functional principles are at play (Section 2). Similar principles may operate within species, and contribute to explaining differences in life history strategies between populations or even individuals. Following this line of reasoning, researchers in biology, anthropology, and evolutionary psychology have argued that within-species individual differences are partly organized along a fast-slow axis of variation. Crucially, this does not just apply to classic life history variables such as fertility and age at maturity, but also to the behavioral and physiological traits hypothesized to mediate the underlying trade-offs. In principle, the fast-slow continuum can help make adaptive sense of the covariation among behavioral and personality traits, their relations with physiological processes, and their developmental antecedents (e.g., early stress; see Belsky et al., 1991; Del Giudice et al., 2015; Ellis et al., 2009; Figueredo et al., 2006; Réale et al., 2010; Wolf et al., 2007). For convenience, I will refer to this set of general ideas as the fast-slow paradigm of individual differences.

Over the last decade, the fast-slow paradigm has become remarkably popular, and has spawned new research subfields and empirical literatures (Nettle \& Frankenhuis, 2019). At the same time, this approach has inevitably come under closer scrutiny, and critics have started to point out problems and unresolved issues. To begin, research based on the fast-slow paradigm has become increasingly self-referential and disconnected from mathematical work on life history evolution (Nettle \& Frankenhuis, 2019). Partly for this reason, tentative hypotheses have been treated as established theory, and researchers have come to rely on overly simplified predictions, without critically examining their assumptions (e.g., Baldini, 2015; Dammhahn et al., 2018; Mathot \& Frankenhuis, 2018; Nettle, 2018; Stearns \& Rodrigues, 2020). On the empirical side, the validity of measures of life history-related traits has been questioned, both in biology and psychology (Copping et al., 2014a, 2014b, 2017; Figueredo et al., 2015; Niemelä \& Dingemanse, 2018; Richardson et al., 2017a, 2017b; Royauté et al., 2018). In humans, some widespread assumptions about the plasticity of life history strategies and the role of early experiences have been criticized, due to gaps in the underlying theory (e.g., Nettle et al., 2013; Del Giudice, 2014a) and contradictory findings from behavior genetics (e.g., Barbaro et al., 2017). The paper by Zietsch and Sidari (2020) is a useful compendium of critical arguments; I provide a concise point-by-point reply to their critiques in the supplementary material (S4).

In sum, it is high time for a reassessment, and this special issue is a great opportunity to move the conversation forward. My goal in this paper is to systematically examine the logic of the fast-slow paradigm. In section 2, I review the basic empirical facts about the fast-slow continuum across species and the main theoretical accounts of its existence. In Section 3, I discuss the move from the level of species to that of individuals, and the theoretical and empirical complications that follow. To anticipate my conclusion, I argue that the fast-slow continuum can be a productive heuristic for individual differences, but needs to be developed further and embedded in a more sophisticated view of life history evolution. This means 
acknowledging the existing theoretical gaps and reconnecting with the biological literature, but also adapting the generic concept of fast versus slow strategies to the specific features of the human ecology. In total, I argue that the field needs to take criticism seriously, and use this opportunity to revise problematic assumptions, drop some bad habits, and start exploring new approaches and ideas.

\section{The Fast-Slow Continuum Among Species}

\subsection{Empirical Patterns}

The term "fast-slow continuum" was coined by Sæther (1987), but the empirical pattern it describes had been noted much earlier (e.g., Pianka, 1970; Tinkle et al., 1970), and initially explained with species differences in $r$-versus $K$-selection (favoring the evolution of faster vs. slower life histories, respectively; see Section 2.2). Species at the fast end of the continuum have high mortality and short lifespans; they mature and start reproduction early, produce small offspring at a fast rate, and show high fertility (at least in mammals and birds; see below for more discussion). Species at the slow end take long to mature and start reproduction, enjoy low mortality rates and long lifespans, and tend to produce few, large offspring at a slow rate. While faster species tend to be smaller and slower species tend to be larger, controlling for body size does not make the continuum disappear, and the overall pattern typically remains very similar (e.g., Del Giudice, 2014b; Stearns, 1983; see below). Fast-slow continua have been documented in mammals (including primates), birds, fish, reptiles, insects, and other animals (e.g., Bakewell et al., 2020; Healy et al., 2019; Jeschke \& Kokko, 2009; Oli, 2004; Promislow \& Harvey, 1990; Ross, 1988; Stearns, 1983); recent comparative studies have found a similar pattern in plants (Rüger et al, 2018; Salguero-Gómez, 2017; Salguero-Gómez et al., 2016).

2.1.1. Is life history variation one-dimensional? There are two common misconceptions about the fast-slow continuum. The first is that, for the continuum to hold, there must be no other major axes of variation in life history traits; that is, life history differences between species must be well described by a one-dimensional model. In reality, it has been clear for decades that there are other important life history dimensions besides fast versus slow (e.g., Stearns, 1983). In mammals, the standard "map" of life history traits is defined by two main axes (Figure 1): a fastslow continuum that typically accounts for $70-80 \%$ of the variance in the traits (about $30-50 \%$ controlling for body size) and a secondary, largely independent axis that explains $10-15 \%$ of the variance (about 20-30\% controlling for body size; see supplementary material S1). This secondary axis distinguishes between species with longer gestations that give birth to larger, precocial offspring (e.g., gazelles) and species with shorter gestations and smaller, altricial offspring that remain dependent for longer (e.g., kangaroos). (Note that the exact nature of the second axis depends on the variables included in the analysis; e.g., Dobson \& Oli, 2007.) A widely cited study by Bielby and colleagues (2007) seemingly failed to recover the structure shown in Figure 1, but the contradictory finding was due to a problem in the analysisspecifically, an inappropriate rotation of the axes in principal components analysis (PCA; see the supplementary material S1 for details). Upon reanalysis, the data showed the same pattern found by Stearns (Del Giudice, 2014b; Figure S1.2). 


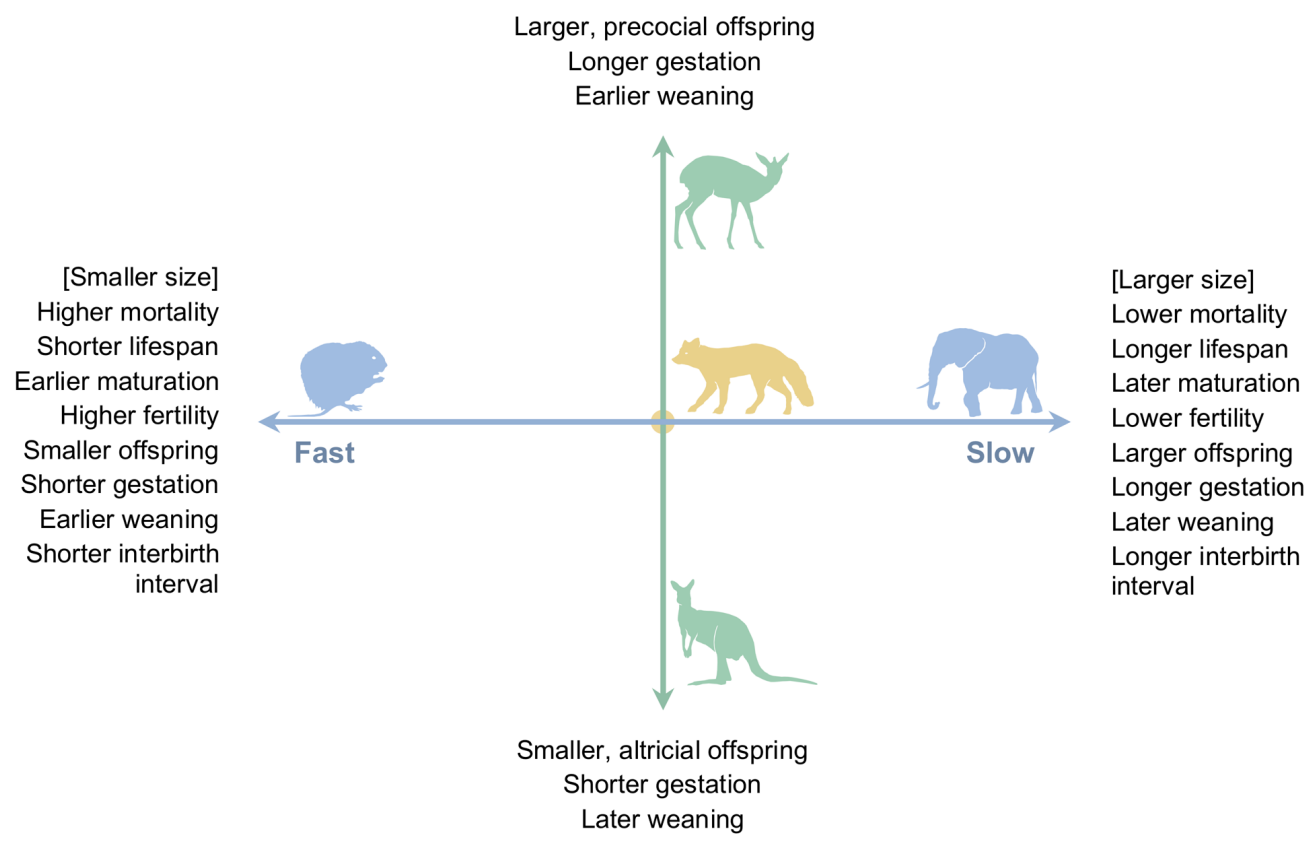

Figure 1. A two-dimensional map of life history traits across mammalian species. Based on Stearns (1983); Promislow \& Harvey (1990); Oli (2004); and the reanalysis of data from Bielby et al. (2007) and Jeschke \& Kokko (2009) presented in the supplementary material (S1; Del Giudice, 2014b).

2.1.2. The role of body size. The second misconception is that the nature of the fast-slow continuum within a given taxonomic group changes dramatically once body size is controlled for, to the point of becoming a conceptually distinct dimension of variation (or disappearing altogether). While the theoretical implications of partialing out body size are far from clear (Section 2.3), this has been interpreted as evidence that the fast-slow continuum is not a robust phenomenon (e.g., Crespi, 2014; Surbey, 2014; Stearns \& Rodrigues, 2020; Zietsch \& Sidari, 2020). The notion that body size has a major impact on the nature of the fast-slow continuum is largely based on the studies by Bielby et al. (2007) and Jeschke and Kokko (2009); it is a misconception because, in both cases, the conclusions of the study are not supported by the data. Again, the problems with the original analyses concern the orientation of axes in PCA; this issue is so pervasive that it deserves a dedicated treatment in the supplementary material (S1).

When the data are properly analyzed, the structure of life history traits turns out to be remarkably robust to the effect of body size (Figures S1.2, S1.3, and S1.4). That said, some taxonomic differences are real and not merely artifactual. Most notably, slower species of fish, reptiles, and insects with larger bodies and longer lifespans also tend to show increased rather than reduced fertility (i.e., larger numbers of eggs; Bakewell et al., 2020; Jeschke \& Kokko, 2009). A plausible reason is that, in these species, egg size is not a strong predictor of offspring quality and survival. As a result, parental investment mainly takes place through enhanced fertility; allocation to increase the number of eggs plays a role similar to allocation to increase offspring size in mammals and birds (see Bakewell et al., 2020; Jeschke \& Kokko, 2009). A recent large-scale analysis of animal life histories by Healy et al. (2019) was largely consistent 
with the existence of a fast-slow continuum, with some exceptions and variations that probably reflect a mixture of true species differences and rotation artifacts (see supplementary material S1).

\subsection{Models of $r / K$ Selection}

The first theoretical explanation of the fast-slow continuum was proposed by Pianka (1970), based on MacArthur and Wilson's model of $r / K$ selection (1967). As I discuss later, these initial contributions suffered from some important limitations. In the $1980 \mathrm{~s}$, the $r / K$ frameworkwith its strong focus on density-dependent selection - fell out of favor, and was largely supplanted by density-independent "demographic" models based on age-dependent schedules of fertility and mortality (see Bassar et al., 2010; Jeschke et al. 2008; Reznick, Bryant, \& Bashey, 2002; Roff, 2002). However, the notion that biologists have stopped using the concept of $r / K$ selection (as suggested for example by Copping et al., 2014b; see also Zietsch \& Sidari, 2020) is an exaggeration. In recent years, $r / K$ models have been significantly updated (Engen et al., 2013; Lande et al., 2009, 2017; Engen \& Sæther, 2016, 2017), and used to develop new ideas about the evolution of fast-slow variation within species (Wright et al., 2019; Section 3).

2.2.1. $r$-selection. The letter $r$ denotes the intrinsic rate of increase of a population, a central parameter in models of life history evolution (Roff, 2002). Selection maximizes $r$ in stable environments when fitness does not depend on population density, or when densitydependence is present but only affects survival (as opposed to fertility) in an age-independent fashion (Dánko et al., 2018; Reznick et al., 2002). In stochastically variable environments, what is maximized is not $r$ but a function of the expected $r$ and its variance; as a result, more variable environments select for faster growth rates (Engen et al., 2013; Sæther \& Engen, 2015). The conditions that lead to $r$ maximization tend to coincide with high mortality rates, low population densities, and (often temporary) phases of unconstrained population growth. Theoretical models predict that $r$-selected species should mature and reproduce early, with high fertility and short lifespans (Engen \& Sæther, 2016, 2017). However, selection to reduce the variance of $r$ may also favor the evolution of plasticity and bet-hedging (Lande et al., 2017; Starrfelt \& Kokko, 2013).

2.2.2. $K$-selection. The letter $K$ indicates the equilibrium size of a population, also known as the environment's "carrying capacity." The idea is that, as a population approaches its maximum size (and hence zero growth), density-dependent effects on fitness become stronger as individuals compete more intensely for resources and reproductive opportunities. As a result, $r$ tends to zero, and the expected lifetime reproductive success $R_{0}$ becomes a more appropriate measure of fitness. Note that this general statement applies if population density affects fertility or age-dependent survival; if density only affects survival independent of age, selection maximizes $r$ as explained earlier (see Dańko et al., 2018). $K$-selection scenarios tend to occur in stable ecologies, which allow populations to reach and maintain high levels of density. Modern $r / K$ models describe a continuum of selection regimes in which environmental variability intensifies selection on $r$, whereas stability and density dependence favor the competitive traits that buffer the detrimental effects of population density (and increase $K$ all else being equal; Engen et al., 2013; Engen \& Sæther, 2016, 2017; Mylius \& Diekmann, 1995; Sæther \& Engen, 2015; see also Dańko et al., 2017, 2018). This does not mean that $K$-selected species will always 
have large populations; for example, if competition occurs via aggression and large bodies, the equilibrium population size may be small in absolute terms (see Wright et al., 2019).

A major problem with Pianka's original $r / K$ framework was the assumption that $K$ selection would favor the opposite traits of $r$-selection-late maturation, delayed reproduction, low fertility, and a long lifespan. But things are not that simple: even under strong density dependence, selection may favor early reproduction and a shorter lifespan if unavoidable or extrinsic mortality ${ }^{1}$ is high (Dańko et al., 2017, 2018). Other complications arise from the way in which density dependence and stochasticity jointly affect survival and reproduction (see Bassar et al, 2010; Reznick et al., 2002). In sum, density dependence does not favor a unique pattern of life history traits, and predictions may vary depending on the details of a species' ecology. This may explain the inconsistent findings that contributed to the downfall of $r / K$ models in the $1980 \mathrm{~s}$ (see Jeschke et al., 2008; Roff, 2002; Stearns, 1992).

2.2.3. $\mathbf{r} / K$ models and the fast-slow continuum. Early $r / K$ models postulated a direct trade-off between $r$ and $K$ without a compelling rationale (Jeschke et al., 2008; Reznick et al., 2002; Stearns, 1992). More recent $r / K$ models are more explicit about the links between density dependence and specific life history traits; but they still suffer from a similar problem, as the functional basis of key trade-offs is left unspecified or described in ways that remain open to multiple interpretations. For example, Engen and Sæther (2016) assumed that phenotypes characterized by early reproduction and faster growth rates are more affected by population density; based on this assumption, they predicted that more variable environments will select for earlier maturation and reproduction. Engen and Sæther framed this trade-off as an alternative to standard models of allocation to current versus future reproduction. Wright et al. (2019) based their work on the same model, but argued that the current-future reproduction trade-off is the functional basis for the trade-off between a faster growth rate (achieved through early reproduction) and the ability to withstand the detrimental effects of population density (achieved through delayed reproduction and investment in competitive traits). In conclusion, modern $r / K$ models can explain some important aspects of the fast-slow continuum; however, they require additional functional assumptions and still do not provide a complete, first-principles account of the observed covariation patterns.

\subsection{Other Theoretical Models}

As density-independent models based on demographic schedules displaced the original $r / K$ framework, the field moved away from all-encompassing theories and toward the exploration of narrower, well defined trade-offs (Jeschke et al., 2008; Roff, 2002). Accordingly, the demographic approach to life history evolution has not provided (or sought) a unified explanation of the fast-slow continuum. Still, key theoretical results point to extrinsic mortality

\footnotetext{
${ }^{1}$ The exact meaning of "extrinsic mortality" varies somewhat between disciplines. In evolutionary psychology and anthropology, "extrinsic" is used to mean that mortality is unavoidable, i.e., insensitive to the allocation decisions of the organism (e.g., Del Giudice et al., 2015; Ellis et al., 2009; Quinlan, 2007). In theoretical biology, "extrinsic" often has the additional implication that mortality rates are independent of age (e.g., Caswell, 2007). The distinction is important because age-independent changes in mortality can only affect life history evolution if fertility and/or juvenile mortality are density-dependent. In and by itself, the level of extrinsic (age-independent) mortality experienced by an organism has no effect on the evolution of life history traits (André \& Rousset, 2020; Dańko et al., 2017, 2018; Reznick et al., 2002).
} 
as a driver of earlier maturation/reproduction and lower investment in offspring quality (summarized in Del Giudice et al., 2015). The role of stochastic (unpredictable) variability in mortality rates is more complex; models indicate that stochasticity in adult survival should typically select for early reproduction, whereas stochasticity in juvenile survival delays reproduction and favors the evolution of bet-hedging (Charlesworth, 1994; see Ellis et al., 2009).

From the standpoint of $r / K$ models, extrinsic mortality (for example via predation or disease) reduces population density and hence tends to increase $r$-selection; even under density dependence, high mortality rates select for earlier reproduction and a shorter lifespan. Similarly, environmental variability can be expected to favor faster growth rates and earlier reproduction (Section 2.2). While life history models admit all sorts of complications and exceptions (Roff, 2002), these convergent predictions suggest the tentative generalization that extrinsic mortality and stochasticity play important roles in the fast-slow continuum (Del Giudice et al., 2015; see also André \& Rousset, 2020).

From a different perspective, allometric models seek to derive large-scale life history patterns from basic energetic constraints related to growth and body size. For instance, the model advanced by Charnov (1991) is able to reproduce the general pattern of correlations observed among life history traits (controlling for body size), by assuming a stable population, densitydependent juvenile mortality, and certain allometric relations between adult size and age of maturity (see also Charnov \& Berrigan, 1993; Charnov et al., 2013). Another well-known example is the metabolic theory of ecology by Brown et al. (2004; Brown \& Sibly, 2006; Sibly \& Brown, 2007). The core idea is that the metabolic rate of an organism scales as a constant power of its body mass, and in turn determines the pace of other biological schedules - from reproductive rate to age at maturity and longevity. The main stumbling block for the metabolic theory is the fact that the fast-slow continuum remains largely intact after body size is controlled for (Section 2.1). This suggests that broad patterns of covariation between life history traits may owe more to selection than to simple metabolic constraints (for recent data in this direction, see Boyce et al., 2020; Malerba \& Marshall, 2019). Moreover, body size is implicated in all sorts of trade-offs: a larger body can reduce predation risk, enhance mating success, buffer the effects of competition in high-density ecologies, and so forth (see Brown \& Sibly, 2006). Controlling for body size in comparative analyses also removes these adaptive effects, raising the question of whether mass-corrected correlations make sense from a theoretical standpoint (see Jeschke \& Kokko, 2009; Roff, 2002).

\section{The Fast-Slow Continuum Among Individuals}

In this section I take a close look at the logic of the fast-slow paradigm. I do so in four connected steps. First, I discuss the move from between-species patterns of life history variation to within-species patterns that resemble the fast-slow continuum (Section 3.1). Next, I consider the move from demographic traits such as fertility, longevity, and age at first reproduction to the behavioral and physiological traits that are the main focus of the fast-slow paradigm (Section 3.2). Third, I review the genetic and developmental mechanisms that may produce individual differences in life histories, as well as adaptive covariation among traits (Section 3.3). Fourth and finally, I address some factors that may complicate or obscure trait correlations at the betweenindividual level (Section 3.4). 


\subsection{The Functional Structure of Life History Strategies and the Ecological Gambit}

All the life history traits discussed so far show considerable variation within species - not just among populations but also among individual organisms. The hypothesis at the heart of the fast-slow paradigm is that the structure of individual differences in life history traits resembles in important ways the structure of variation across species. This hypothesis depends on what I will call the ecological gambit: the working assumption that relations observed at the group level will hold at the individual level, for similar functional reasons ${ }^{2}$. If the assumption is supported, it offers a useful heuristic for studying individual differences and facilitates empirical progress. The ecological gambit is analogous to other heuristics routinely adopted in evolutionary biology, most notably the phenotypic gambit (the working assumption that the genetic architecture does not constrain which phenotypes can evolve in the long run; Grafen, 1984) and the behavioral gambit (the working assumption that psychological mechanisms do not constrain the expression of adaptive behavior; Fawcett et al., 2013). While these often prove to be reasonable starting assumptions, they may or may not apply to any specific case and can lead to errors if applied unthinkingly (see Nettle et al., 2013). The evil twin of the ecological gambit is the ecological fallacy - the assumption that group-level relations automatically or necessarily hold at the between-individual level (Robinson, 1950; see also Pollet et al., 2014). Without a detailed understanding of the mechanisms that generate covariation within and between populations, it is hard to anticipate whether (and to what extent) the gambit is likely to be productive. In general, the gambit becomes riskier if causal factors that affect multiple variables of interest are known to operate at one level of analysis, but not at the other (see Pollet et al., 2014). While there is no guarantee that the gambit will succeed in any particular case, there may be conceptual reasons to treat the assumption of cross-level similarity as more (or less) biologically plausible.

3.1.1. General arguments for cross-level consistency. There are both general and specific arguments that lend initial plausibility to the ecological gambit for life history strategies. On the general side, adaptive life history strategies require the integration of multiple traits, and often show coordinated plasticity to environmental conditions (Braendle et al., 2011; Roff, 2002). For this reason, life history traits can be expected to be developmentally and genetically correlated, with extensive pleiotropic effects (more on this in Section 3.3); this makes it more likely that within-species patterns of covariation also exist among populations and closely related species (Peiman \& Robinson, 2017; see Réale et al., 2010). Moreover, developmental plasticity and pleiotropy bias the distribution of individual phenotypes, channeling the effects of mutations and environmental changes along the existing reaction surface ${ }^{3}$ (often with adaptive consequences). The resulting developmental biases impose directionality on evolutionary trajectories, so that patterns of divergence between species and populations tend to align with correlations between individuals (Figure 2a; Draghi \& Whitlock, 2012; Ellis et al., 2009; Uller et al., 2018; West-Eberhard, 2003).

\footnotetext{
${ }^{2}$ Note that, in this paper, I focus specifically on patterns of differences and covariation between individuals, and do not address the topic of variation within the same individual over time (e.g., Dingemanse \& Dochtermann, 2013).

${ }^{3}$ A reaction surface is the multivariate generalization of a reaction norm (see Pigliucci, 2005). A reaction norm is the function that describes how different phenotypes develop in response to different values of a single environmental variable; a reaction surface generalizes this concept to multiple dimensions of the environment.
} 

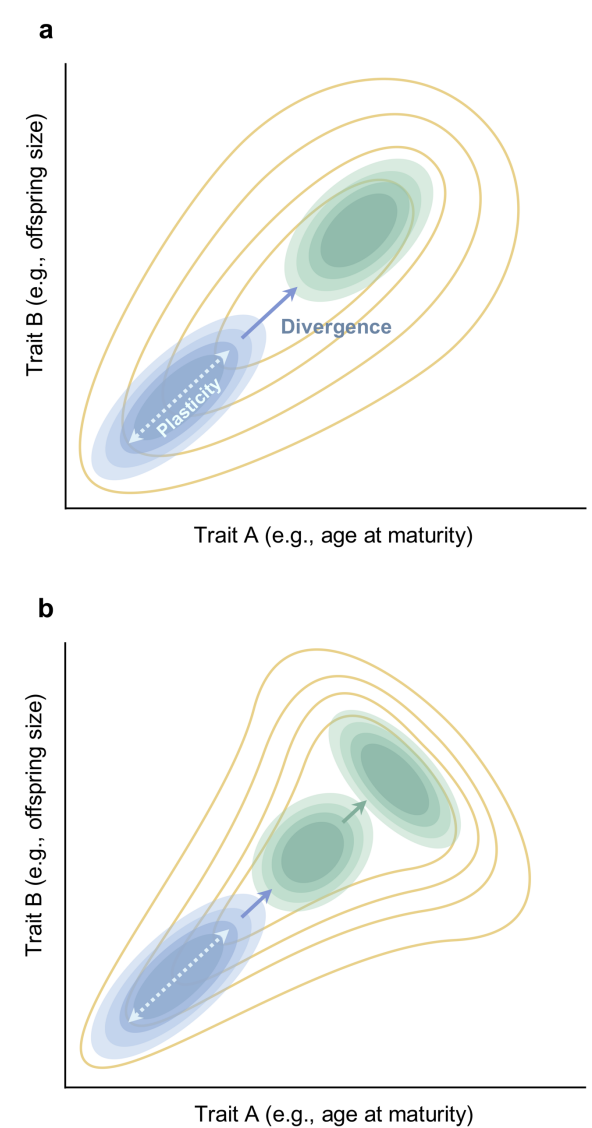

Figure 2. Patterns of evolutionary divergence and the ecological gambit. In the ancestral population (lower left), developmental plasticity aligns with the genetic correlation between the two traits. Developmental and genetic correlations orient the initial response to selection along the axis of plasticity and facilitate adaptive evolution. In panel (a), the fitness landscape for the descendent population (upper right) maintains its features as the population diverges under selection. The trait correlation between populations mirrors the correlation within each population, and the ecological gambit is successful. In panel (b), the fitness landscape changes dramatically as the descendent population diverges; despite the initial bias produced by the genetic and developmental structure, selection eventually reverses the trait correlation. As a result, the ecological gambit succeeds in the ancestral population but fails in the descendent population.

3.1.2. The role of basic life history trade-offs. To be sure, the arguments I just reviewed are merely suggestive: for example, even strong genetic and developmental correlations can break down relatively quickly under intense selection (e.g., Chippindale et al., 2003; Conner et al., 2011). The hypothesized similarity between within- and between-species patterns (e.g., Dammhahn et al., 2018) seems to require sufficient constancy in the underlying fitness landscape (Figure 2). This is where the notion of basic life history trade-offs comes into play. Fundamental trade-offs such as those between current and future reproduction and between quality and quantity of offspring shape the allocations of individual organisms; but since population-level traits are averages of individual outcomes, the same trade-offs should be reflected at both the within- and the between-population levels (even if not identically). In other words, basic trade- 
offs contribute to defining a common fitness landscape for life history strategies across levels (see Wright et al., 2019). Another piece of the puzzle is that trade-offs are not functionally independent from one another. One of the key benefits of delaying reproduction is the ability to produce higher-quality offspring, which implies a functional link between the current-future reproduction trade-off and the quality-quantity trade-off (e.g., Wright et al., 2019). And for animals that increase offspring quality through parental care, the mating-parenting trade-off is going to overlap significantly with that between quality and quantity (see Del Giudice et al., 2015).

Even admitting exceptions and complications (Section 3.4), these functional links should tend to generate predictable relations among multiple life history traits, both within and across species. A much harder question is whether the same ecological factors that select for certain life history traits at the population/species level will also maintain genetic variation in the same traits among individuals, or prompt their development through mechanisms of adaptive plasticity. If one considers specific trade-offs, there are examples of developmental models that mimic the predictions of population-based demographic models. For instance, Berrigan and Koella (1994) found that the optimal plastic strategy involves early maturation in response to high juvenile mortality, and delayed maturation in response to energetic scarcity.

That said, there is an obvious theoretical gap regarding the fast-slow continuum as a whole. This is not surprising: as I noted in Section 2, even the fast-slow continuum across species is still waiting for a widely accepted formal explanation. The closest attempt so far is the recent verbal model by Wright et al. (2019). Drawing on recent versions of the $r / K$ model (Engen et al., 2013; Engen \& Sæther, 2016, 2017), these authors assumed that each species experiences a characteristic average level of density-dependent selection, leading to the emergence of a fastslow continuum across species. On top of this average pattern, however, the intensity of density dependence within each species can be expected to fluctuate over time as populations grow and shrink. Wright and colleagues argued that fluctuating density-dependent selection explains the maintenance of individual variation on the fast-slow continuum (in combination with frequencydependent selection within populations); crucially, the same process would also account for the existence of similar covariation patterns at the species and individual level of analysis.

None of the above implies that the between-individual fast-slow continuum of a given species should be identical to that of another, especially if distantly related. Even the comparative fast-slow continuum shows some meaningful differences among taxonomic groups, so a certain degree of variability is to be expected. For example, the strength of the functional link between offspring quality and future reproduction is likely to vary across species, and correlations between the relevant life history traits (e.g., age at maturity and offspring size) should change accordingly. In the recent biological literature on pace-of-life syndromes (POLS), some authors have defined the fast-slow continuum narrowly in terms of the current-future reproduction trade-off, partly to increase the precision of theoretical predictions (e.g., ArayaAjoy et al., 2018; Dammhahn et al., 2018; Mathot \& Frankenhuis, 2018). At the same time, the density-based model of POLS evolution by Wright et al. (2019) has brought the quality-quantity trade-off back into focus, thanks to its emphasis on density dependence. My point is that the trade-off between current and future reproduction should not be considered in isolation: tradeoffs between offspring quality and quantity or between mating and parenting can be just as 
important, particularly for males - who can often make up for delayed reproduction by mating with more or higher-quality partners - and for long-lived species with flexible reproductive schedules and extended parenting, including humans (supplementary material S4).

To sum up: critics (e.g., Baldini, 2015; Schmitt, 2019; Stearns \& Rodrigues, 2020; Zietsch \& Sidari, 2020) are right to point out that patterns observed between species or populations cannot be automatically generalized to individuals within a species; the ecological gambit is only a working assumption, and the ecological fallacy is always a danger. In the case of life history strategies, there are arguments that lend face plausibility to the idea of a fast-slow continuum at the between-individual level, with the provision that the details of how trade-offs interact - and life history traits correlate - are likely to show some variability across species. While this is encouraging for proponents of the fast-slow paradigm, these arguments have not been formalized in detail, and must be regarded as preliminary until then. Also, there is an important distinction between the weaker hypothesis that individual life history strategies will be partly described by a fast-slow continuum, and the stronger hypothesis that ecological factorssuch as mortality, population density, and unpredictability - affect the development of individual strategies in the same way as they shape the evolution of strategies at the level of populations and species.

3.1.3. Baldini's critique. The role of ecological factors at different levels of organization is the topic of a widely read preprint by Baldini (2015), which in the words of Nettle (2018) has quickly become a "subcultural classic." The mathematical models in the paper are used to make two main points. The first is that various aspects of environmental harshness (e.g., mortality rates) can have different and even contrasting effects on the evolution of population-level life history traits (e.g., age at maturity), conditional on other factors such as the presence of densitydependent selection. While this is not a novel insight, it is true that density dependence moderates the impact of mortality on life history evolution (Section 2.2). This point has often been neglected in the human literature. However, there are exceptions: Ellis et al. (2009) discussed density dependence in considerable detail, and Sng and colleagues have started to explore the relations between population density and life history-related traits (Sng \& Ackerman, 2020; Sng et al., 2017). A problem with Baldini's model — and a likely source of confusion about its implications - is that "extrinsic mortality" is defined so as to be preventable by investing resources in survival (with increasing marginal returns). This is not how extrinsic mortality is usually conceptualized (see Footnote 1); some counterintuitive results of the model follow directly from this inconsistency (André \& Rousset, 2020).

The second major point of Baldini's paper is that the optimal plastic response to a given ecological factor (e.g., mortality) at the individual level does not necessarily mirror the evolutionary response of the population to the same factor (e.g., Kawecki \& Stearns, 1993). This is a valid concern that has not been adequately addressed in the human literature. However, Baldini's main result regarding the optimal response to variation in mortality depends on the same idiosyncratic definition of extrinsic mortality discussed above (André \& Rousset, 2020).

\footnotetext{
${ }^{4}$ Another questionable aspect of the model is that the fertility rate is assumed to be directly proportional to the age at maturity, so that — all else being equal — later-maturing organisms produce more offspring per year, without any quality-quantity trade-off. This assumption runs counter the empirical pattern observed in mammals and birds, and may explain some counterintuitive predictions of the model in the density-independent scenario.
} 
Moreover, the model assumes that ecological conditions vary across space but not over time, so that the population effectively contains multiple independent sub-populations at any given time. When the environment fluctuates over time, the entire population undergoes the same changes in conditions and the logic of Baldini's model does not apply. (In most realistic scenarios, spatial and temporal variation coexist; see Starrfelt \& Kokko, 2013.) This is relevant because some models of individual variation in life history strategies (e.g., Del Giudice, 2012; Wright et al., 2019) are explicitly based on temporally variable selection (Section 3.3).

\subsection{The Role of Behavior and Physiology in Life History Strategies}

In a narrow sense, life history strategies are patterns of allocation among fitness components; within the constraints of various trade-offs, these allocations determine the life history traits of individuals and species (summarized in Del Giudice et al., 2015). The basic life history traits are age at first reproduction, age-specific fertility, and age-specific mortality; these are also called direct fitness traits (Roff, 2002) because they are sufficient to calculate $r$ and $R_{0}$, as well as other demographic traits such as the expected fertility rate and longevity. However, life history strategies do not exist in an abstract theoretical world: what actually mediates allocations and trade-offs are the organism's behaviors, physiological mechanisms, and physical characteristics. "Growth" is the outcome of a causal pathway that includes feeding and foraging behaviors, plus the metabolic and hormonal mechanisms that convert energy into tissues.

"Survival" necessitates the activity of myriad processes, from immunity and other forms of body maintenance to the behavioral mechanisms that mediate fear, risk-avoidance, disgust, and so on. Besides its many physiological requirements, "reproduction" is brought about by a constellation of complex behaviors that may include courtship, parental behaviors, and pair-bonding. This broader, process-oriented view of life history strategies is illustrated in Figure 3.

From this perspective, life history strategies are expressed as combinations of coadapted behavioral, physiological, and morphological traits (Braendle et al., 2011). Ultimately, these traits contribute to fitness through their effects on basic life history parameters - age at first reproduction, fertility, and mortality - within the constraints set by allocation trade-offs (Figure $3)$. This is the foundation of the POLS concept and of the fast-slow paradigm more broadly: if life history strategies can be arranged on a fast-slow continuum within a species, this will partly explain the covariation among the behavioral and physiological traits that mediate the underlying trade-offs (e.g., Dammhahn et al., 2018; Figueredo et al., 2006; Réale et al., 2010). I suggest to refer to these traits as life history-related (or some equivalent label) to mark the distinction with demographic life history variables such as fertility and mortality. More specifically, life historyrelated traits should (a) be intra-individually stable enough to be treated as individual differences variables; (b) covary with basic life history traits and/or other outcomes of life history allocations (e.g., number of sexual partners, age of reproduction); and (c) plausibly contribute to mediating those allocations, or at least function as proxies of traits that do. As I discuss in section 3.4, patterns of covariation may be complex and context-dependent, and the causal role played by any given trait may be quite indirect. The point is that putative life history-related traits must be validated against tangible outcomes to avoid circular reasoning (Copping et al., 2017; Zietsch \& Sidari, 2020). 


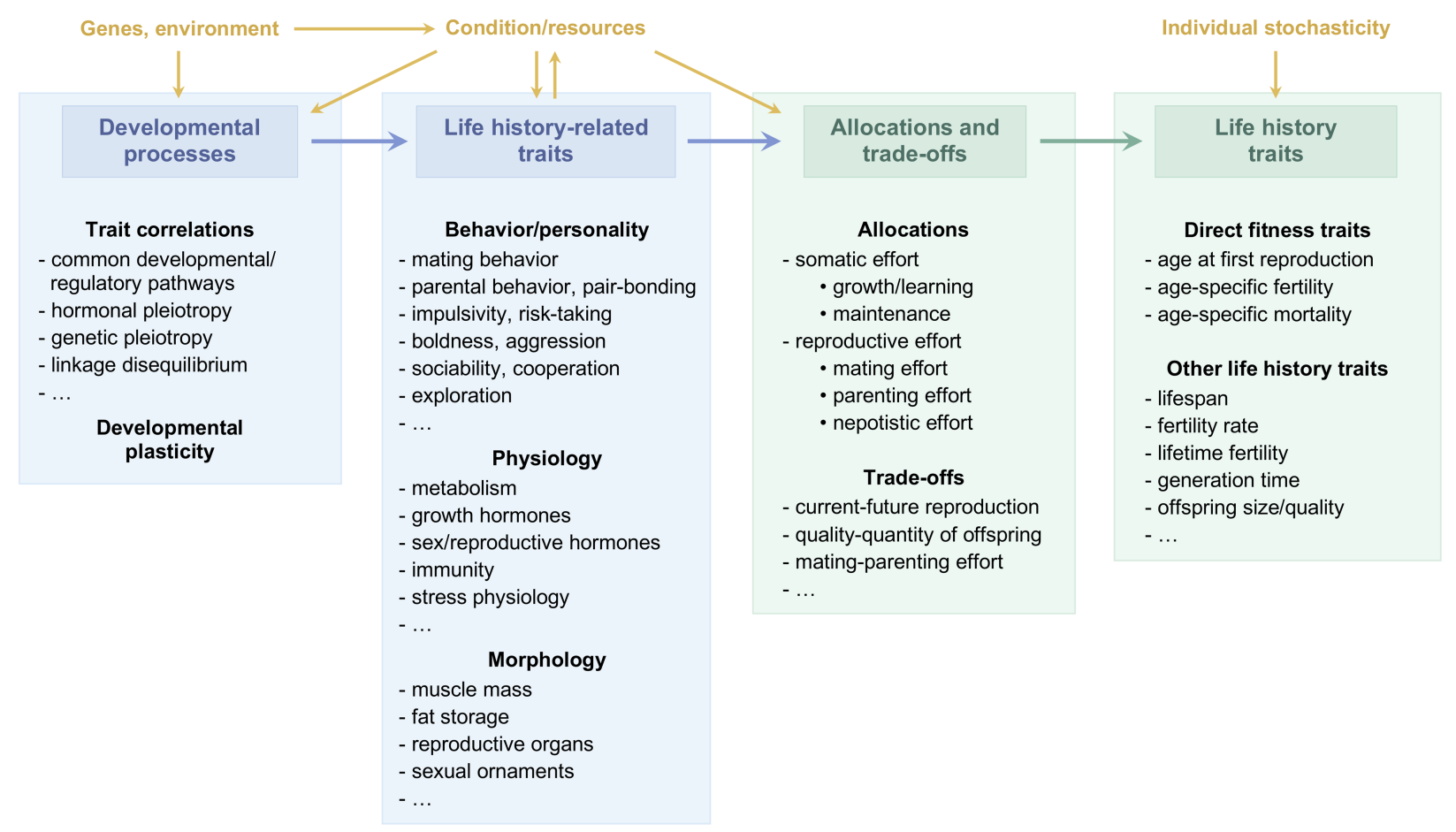

Figure 3. A process view of life history strategies. Note how individual differences in condition and resources influence the development and expression of life history-related traits, are affected in return, and partly determine the efficiency and outcome of allocations.

Figure 3 helps to make an important but sometimes overlooked point: life history strategies are formally defined at the level of allocations and trade-offs, not at that of specific behavioral and physiological traits. In many if not most instances, the same behaviors may be involved in different allocations, serve multiple functions, or even play functionally opposite roles in different contexts, individuals, or species (Section 3.4). For example, exploratory behaviors may reflect both risk-taking (e.g., risking predation and injuries while searching for food or mates) and risk-avoidance (e.g., searching the surroundings for cues of hidden dangers). Similarly, the "competitive" traits favored in high-density ecologies may take a number of rather different forms - from large body size and aggression to sociability and cooperation (Ellis et al., 2009; Wright et al., 2019).

Neglect of this crucial distinction has been a major problem in research on animal POLS, and has likely contributed to the many inconsistent findings in this literature (Royauté et al., 2018; Smith \& Blumstein, 2008). When Réale et al. (2010) put forward a list of plausible life history-related traits (e.g., high activity and high sympathetic activity in "fast" individuals), they also warned that the list was tentative and unlikely to be widely applicable across species. Unfortunately, the list was quickly reified; investigators went on to test the suggested correlations in a variety of species, usually without testing key assumption about the functional role of the traits they measured. This is especially problematic given the dearth of formal models linking life history trade-offs to the evolution of specific behavioral/physiological traits 
(Dammhahn et al., 2018; Mathot \& Frankenhuis, 2018). More recent work has started to address these questions more explicitly (e.g., Fenneman \& Frankenhuis, in press).

\subsection{Mechanisms of Trait Variation and Covariation}

3.3.1. Genetic variation. To a first approximation, the genetic architecture of life history strategies is similar in humans and nonhuman animals. The heritability of life history traits such as longevity, age at first reproduction, and lifetime reproductive success tends to range between 10\% and 40\% (Briley et al., 2017; Kosova et al., 2010; Madrigal et al., 2003; Roff, 2002; Stearns et al., 2010; Tropf et al., 2015), in line with the stochastic nature of these outcomes (Section 3.4). Likewise, the heritability of personality is $40-50 \%$ in adult humans and about $50 \%$ across species (Briley \& Tucker-Drob, 2017; Dochtermann et al., 2015; Vukasović \& Bratko, 2015). The distribution of genetic effects also follows a common template: with few exceptions (see Weitkamp \& Keller, 2019), behavioral traits tend to be highly polygenic, with many loci of small effect and few (if any) loci with common alleles of large effect (Chabris, 2015; Sella \& Barton, 2019; Weitkamp \& Keller, 2019).

The two main processes that can maintain genetic variability in traits under selection are mutation-selection balance and balancing selection (see Gangestad, 2011; Keller, 2018; Sella \& Barton, 2019). Deleterious mutations contribute substantially to individual condition (Section 3.4; Zietsch \& Sidari, 2020); those with large detrimental effects are quickly purged by selection and hence remain rare, whereas slightly deleterious alleles can persist at high frequencies for a long time (Keller, 2018). In humans, rare and structural variants (e.g., copy number variations) may account for $50 \%$ or more of the genetic variance of traits such as height, intelligence, and neuroticism (Hill et al., 2018; Nolte et al., 2019; Wainschtein et al., 2019).

The role of mutations is not controversial, even if it has not always received the attention it deserves in the life history literature. The main point of contention is whether common genetic variation in life history-related traits is partly maintained by balancing selection - that is, selection that systematically changes direction across individuals, space, or time. Fluctuating selection and frequency-dependent selection are specific kinds of balancing selection. In animals with complex social systems, frequency-dependent selection can occur via social "nichepicking," whereby individuals seek out roles that match their phenotypes.

Critics argue that balancing selection should yield genetic architectures in which highfrequency alleles account for most of the variance - a pattern inconsistent with the empirical data for most quantitative traits (Verweij et al., 2012; Zietsch \& Sidari, 2020). Genomic studies have found relatively few loci matching the expected signatures of balanced polymorphisms (but see Bitarello et al., 2018); accordingly, many authors regard balancing selection as a marginal phenomenon, despite ample evidence of variable selection in nature (see Messer et al., 2016; Thompson, 2013). Skepticism is even stronger for hypotheses that involve temporal fluctuations, which - in contrast to spatial fluctuations - are often believed to be ineffective in maintaining genetic variation (Messer et al., 2016). This is important because fluctuations over time could plausibly generate individual differences along a fast-slow axis. For example, Wright et al. (2019) recently proposed fluctuating density-dependent selection as a general explanation for individual variation in POLS. But there are other features of the environment that may play a 
similar role. For example, I have argued that temporal fluctuations of the sex ratio determine shifts in the costs and benefits of life history allocations, particularly between mating and parenting (Del Giudice, 2012).

As it turns out, these widespread ideas about balancing selection and temporal variation have been substantially revised in the theoretical literature (see Del Giudice, 2012; Penke \& Jokela, 2016). To begin, it has become clear that balancing selection takes an exceedingly long time to generate its classic signatures. In realistic scenarios involving highly polygenic traits, balancing selection is not expected to produce common alleles of large effect, and can be very hard to distinguish from recent positive selection or even neutrality (Connallon \& Clark, 2013; Fijarczyk \& Babik, 2015). Likewise, contemporary models show that temporal fluctuations can be quite effective in maintaining genetic variation, particularly in species with overlapping generations (including humans; Bertram \& Masel, 2019; Ellner \& Sasaki, 1996; Yamamichi \& Hoso, 2016). A more detailed overview of these issues can be found in the supplementary material (S2).

3.3.2. Developmental plasticity. It should often be adaptive for organisms to adjust their life history allocations based on cues about the state of the environment and/or their own condition. However, sensitivity to the environment can also be detrimental - for example because it exposes individuals to dysregulation and mismatch - and adaptive plasticity is often assumed rather than convincingly demonstrated (e.g., Hendry, 2015). Whether plasticity is favored depends on a number of factors including the reliability of cues, the stability of the environment across an individual's lifetime, and the predictability of future states (Nettle \& Bateson, 2015). The same factors determine the optimal balance in the integration of genetic and environmental information (McNamara et al., 2016). Unfortunately, for many organismsincluding humans - the relevant statistical features of the ecology are unknown or still poorly understood (Frankenhuis et al., 2019).

In the classic view of plasticity, the developing organism employs early life cues (e.g., nutrition, exposure to stressors) to forecast the future state of the environment, and match its phenotype to the predicted state ("external" predictive-adaptive response; see Bateson et al., 2014). An alternative possibility - especially when the environment is unpredictable or changes too quickly relative to the organism's lifespan - is to use early cues to forecast the future state of the soma instead of that of the environment ("internal" predictive-adaptive response; see Nettle \& Bateson, 2015; Nettle et al., 2013). For example, early stress may work as a cue of a dangerous environment, but may also cause somatic wear and tear, which in turn may reduce the individual's expected survival (e.g., Chang et al., 2019a; Dunn et al., 2019; Rickard et al., 2014). There is urgent need for realistic models; as I noted in Section 3.1, the assumption that plastic responses at the individual level should mimic evolutionary responses at the population level is not based on formal theory, and cannot be taken for granted.

Another urgent question in the human literature is how to reconcile theories of adaptive plasticity with the findings of behavior genetics. For most life history and life history-related traits, twin studies show a small to negligible role of shared environmental factors - that is, factors that act consistently on siblings from the same family, increasing their similarity (e.g., Bartels et al., 2003; Briley et al., 2017; Briley \& Tucker-Drob, 2017; Franz et al., 2010; Morris et 
al., 2011; Ouellet-Morin et al., 2009; Polderman et al., 2015; Tropf et al., 2015; Tucker-Drob et al., 2017). In most cases, their estimated contribution in adulthood is less than $10 \%$ of the variance (for some notable exceptions see Kendler et al., 2019). On the face of it, this is a puzzling pattern. If developmental processes respond to life history-relevant factors such as mortality and resource availability, and if parental behaviors and family stress act as cues during childhood (Belsky et al., 1991; Chisholm, 1993, 1999; Ellis et al., 2009), what explains the surprisingly small impact of growing up in the same family?

One possibility is that developmental plasticity is mostly nonadaptive, or does not respond to the particular factors emphasized in the fast-slow paradigm (Zietsch \& Sidari, 2020). Instead, the apparent effects of family variables may be mediated by shared genetic factors - a suspicion reinforced by the lack of genetic controls in most developmental studies (e.g., Barbaro et al., 2017). However, this is not the only possible explanation. For example, individuals may systematically differ in their level of plasticity, as postulated by theories of differential susceptibility (see Belsky \& Pluess, 2013; Ellis \& Del Giudice, 2019; Ellis et al., 2011). Under some conditions, this type of $\mathrm{G} \times \mathrm{E}$ interaction may account for the results of twin studies, and even accommodate a major role of the shared environment in shaping early plasticity (Del Giudice, 2016). Natural experiments have shown that children respond to traumatic events (e.g., earthquakes) with accelerated maturation and increased reproductive effort (Lian et al., 2018; Pesonen et al., 2008); but the impact of weaker, common stressors that vary across families is still unclear. The tension between developmental models and behavior genetic findings is a major challenge that needs to be recognized and addressed.

For reasons of space, I will not discuss developmental factors further in this paper. For some recent (but not genetically informative) studies that relate childhood stress to life historyrelated traits in adolescence and adulthood see Chang and Liu (2018), Chang et al. (2019a, 2019b), Deater-Deckard et al. (2019), Holdsworth \& Appleton (2020), Mell et al. (2018), Sear et al. (2019), and Szepsenwol et al. (2017).

3.3.3. Mechanisms of covariation. From a genetic perspective, trait correlations can arise in a number of ways (Saltz et al., 2017). Correlational selection for specific combinations of traits, assortative mating, and other forms of social selection may produce nonrandom associations between alleles (linkage disequilibrium). As noted by Zietsch and Sidari (2020), correlations due to linkage disequilibrium are usually volatile and temporary (Roff \& Fairbairn, 2007; Saltz et al., 2017; see also Revell, 2007). However, it has been suggested that persistent correlational selection may favor the evolution of regulatory loci that produce the same association through pleiotropic effects (Peiman \& Robinson, 2017; Roff \& Fairbairn, 2007). Also, fluctuating selection that acts simultaneously on multiple traits (co-selection) can favor the evolution of genetic correlations via pleiotropy (Pavličev et al., 2011; see also Pavličev \& Cheverud, 2015).

Especially when they get entrenched in developmental processes, pleiotropic effects can be stable and fairly robust. Deleterious mutations can also have broad pleiotropic effects, and tend to produce positive correlations among traits that reflect individual condition or quality (Section 3.4). In principle, genetic correlations among traits could be suppressed or reversed at the phenotypic level by environmental factors acting in the opposite direction. In practice, 
phenotypic correlations generally have the same sign of the corresponding genetic correlations, and tend to be only somewhat smaller in magnitude (Dochtermann, 2011; Kruuk et al., 2008; Roff, 1996; Sodini et al., 2018). The implication is that genetic and environmental effects tend to act in the same direction; this suggests a confluence of adaptive plasticity and balancing selection on the same phenotypic traits, and/or a confluence of mutations and environmental disturbances on the same developmental mechanisms.

When pleiotropic correlations evolve as a result of trade-offs, they are usually produced by multiple loci of small effect rather than "master switch" genes (Saltz et al., 2017). Zietsch and Sidari (2020) cite the absence of large-effect pleiotropic alleles to dismiss the possibility of adaptive trait coordination via genetic mechanisms. It is entirely possible to envision polygenic regulatory mechanisms that receive inputs from multiple genetic and environmental sources, and adaptively coordinate the expression of suites of traits. In fact, this is precisely how endocrine systems work (Ketterson et al., 2009; Vitousek \& Schoenle, 2019). Hormones are agents of biological coordination: they regulate cell activity and gene expression across multiple tissues and achieve integration among physiological, morphological, and behavioral traits (Cox, McGlothlin, \& Bonier, 2016). The ability of hormones to influence many target tissues at once has been called hormonal pleiotropy; in addition, hormones often act as physiological mediators of pleiotropy at the genetic level (Dantzer \& Swanson, 2017; Ketterson \& Nolan, 1999; Ketterson et al., 2009).

The major endocrine systems that regulate life history allocations are remarkably conserved across species (Vitousek \& Schoenle, 2019). For instance, testosterone and other androgens regulate trade-offs between mating, parenting, and survival in vertebrates (Hau \& Wingfield, 2011; Ketterson et al., 2009). In both vertebrates and invertebrates, insulin-like growth factor 1 (IGF-1) regulates trade-offs between growth, fertility, and survival (Gerish \& Antebi, 2011; Swanson \& Dantzer, 2014; Vitousek \& Schoenle, 2019). Other vital coordination roles among growth, metabolism, immunity, reproduction, and behavior are played by the HPA axis (Crespi et al., 2013; Ellis \& Del Giudice, 2014; Hau et al., 2016), in concert with the hypothalamic-pituitary-gonadal (HPG) and hypothalamic-pituitary-thyroid (HPT) axes, the IGF1 signaling system, and pathways involving prolactin, oxytocin, vasopressin/vasotocin, and immune cytokines (Del Giudice, 2018; Ellis, 2013; Lancaster \& Sinervo, 2011; Vitousek \& Schoenle, 2019). By virtue of their biological function, hormonal mechanisms are both plastic and highly pleiotropic (Vitousek \& Schoenle, 2019). Much of the evolution of endocrine systems takes places through changes in the receptivity and gene expression of individual tissues, rather than at the level of the central signaling molecules (Dantzer \& Swanson, 2017). Hence, their genetic basis is going to be quite complex, with many layers of upstream and downstream regulation and different roles for the same genes across organs and cell types.

Naturally, hormones are not the only coordination mechanisms that may generate adaptive trait covariation. Neurocognitive mechanisms collect, interpret, and integrate information from the environment; generate predictions about the future and evaluate their validity; and can regulate life history allocations in myriad ways — not just through behavior, but also via top-down regulation of endocrine systems (see Del Giudice et al., 2015). An example in humans is the interplay between social, cognitive, and physiological mechanisms in the process that leads to the start of reproduction (Nettle, 2011). 


\subsection{Patterns of Covariation at the Between-Individual Level}

In Section 3.1 I reviewed reasons why between-species relations among life history traits can be tentatively used as a guide to relations within species (the ecological gambit). I now discuss some factors that, even if the ecological gambit is valid at the functional level of tradeoffs, may attenuate or even reverse the observed correlations between traits. These factors are individual stochasticity in life history outcomes; individual differences in condition and resources; complex functional relations involving behavioral and physiological traits; and measurement error. Without careful consideration of these factors, it is easy to take a literal view of the ecological gambit, and assume that correlations at the between-individual level should be just as strong and clear-cut as those at the level of species. A related expectation is that, when individual life history and/or life history-related traits are subjected to factor analysis or PCA, the results should mirror those of comparative studies - with a strong, general axis of fast-slow variation accounting for a large proportion of trait variance. In my view, these misleading expectations have implicitly shaped the debate around the fast-slow paradigm in humans (e.g., Richardson et al., 2017b; Zietsch \& Sidari, 2020).

3.4.1. Individual stochasticity. Because life history events such as death and reproduction have a stochastic component, life history traits will show a considerable amount of variation even if all the individuals in a population are identical and make the same allocations (Caswell, 2009; Steiner \& Tuljapurkar, 2012). This is the pervasive phenomenon of individual or demographic stochasticity (see Cam et al., 2016; Caswell, 2009; Sæther \& Engen, 2015). As a result of individual stochasticity, between-individual correlations involving life history traits will be necessarily smaller than those between species or populations. The contribution of stochasticity can be estimated using demographic models, and is often the dominant component of variation. For instance, chance accounted for a median $65 \%$ of variance in longevity across invertebrate species (Hartemink \& Caswell, 2018); in a seabird, the proportion was $66 \%$ of longevity and $61 \%$ of lifetime reproductive success (Snyder \& Ellner, 2018).

Individual stochasticity does not affect correlations across species or populations, which are based on group-level averages. But at the between-individual level, it attenuates the correlations among some life history traits (e.g., age at first reproduction and fertility) and puts a low ceiling on the correlations between life history traits and the behavioral/physiological traits that mediate the underlying allocations - even in presence of strong causal effects (e.g., ArayaAjoy et al., 2018). To illustrate, consider a hypothetical species in which $50 \%$ of the variance in longevity is due to chance, and a variable $X$ that predicts individual survival. Even if $X$ accounts for all the explainable variance in survival and is measured without error, its expected correlation with longevity is only .71. If variable $\mathrm{X}$ accounts for $25 \%$ of the explainable variance and is measured with $80 \%$ reliability, the correlation drops to only .32 . Clearly, it is unrealistic to expect strong associations at the between-individual level when traits are substantially affected by stochasticity.

In humans, correlations between personality traits and longevity tend to be less than .10, and correlations between personality and measures of fertility rarely exceed .20 (e.g., Alvergne et al., 2010; Briley et al., 2017; Gurven et al., 2014; Jokela et al., 2011; Međedović et al., 2018; 
Terracciano et al., 2008). These effect sizes necessarily understate the true strength of the functional links between personality and life history. Similar considerations apply to traits such as puberty timing and age at menarche, which are the end results of stochastic physiological processes; but also to competitive outcomes such as mating success and dominance, which are partly determined by luck (see Frankenhuis \& Del Giudice, 2012).

3.4.2. Individual differences in condition and resources. The next factor to consider at the within-species level is individual variation in condition and resources (Figure 3). If some individuals enjoy higher genetic quality (fewer deleterious mutations) or a more favorable environment, they may be able to acquire and allocate more resources to multiple fitness components at once, or achieve fitness-related outcomes more efficiently (e.g., attractive individuals may obtain the same mating success with less mating effort). In other words, they are less constrained in their allocation decisions than worse-off individuals (Reznick et al., 2000; van Noordwijk \& de Jong, 1986). The interplay between resource acquisition and allocation can be quite complex (Roff \& Fairbairn, 2007), and may involve feedback loops in which an organism's life history-related traits (e.g., risk-taking, aggression, foraging behaviors) partly determine its future condition and resources (see Figure 3). Trade-offs may also be loosened when organisms have access to effectively unlimited resources without competition and danger, as is common in laboratory studies (see Reznick et al., 2000; Royauté et al., 2018).

All else being equal, a functional trade-off between competing components should give rise to a negative correlation at the genetic and/or phenotypic level. But when there are sizable differences in condition/resources, the predicted negative correlation can be attenuated, erased, or even reversed (see Reznick et al., 2000; Roff \& Fairbairn, 2007; Stearns, 1989). If individual differences in condition/resources are caused primarily by environmental factors, trade-offs will be masked at the level of phenotypic correlations but manifest in genetic correlations. However, it is often the case that condition and resource acquisition have a genetic component, maintained at least in part through mutation-selection balance (Section 3.3). If so, trade-offs may also be masked at the level of genetic correlations (Houle, 1991; Wilson, 2014).

Simulation studies clearly illustrate how variation in condition/resources can obscure the role of strategic allocations (e.g., Araya-Ajoy et al., 2018). The take-home point is that phenotypic and genetic correlations between life history traits cannot always be taken at face value; methods that work at the level of species and populations (e.g., PCA of uncorrected life history traits; see Araya-Ajoy et al., 2018) may give misleading results when applied to individuals. This is highly relevant to interpreting matrices of genetic correlations among traits, such as the one shown by Zietsch and Sidari $(2020)^{6}$. A pattern of positive genetic correlations

\footnotetext{
${ }^{5}$ If accurate estimates of stochasticity are available, one may get a more realistic sense of the functional associations between traits by adjusting the observed correlation for the impact of chance, not unlike correcting for measurement error. The adjusted correlation would then refer to the relation between the "explainable portions" of the variables.

${ }^{6}$ The matrix in Figure 1 of Zietsch and Sidari (2020) includes a variety of physical symptoms/illnesses, some psychiatric disorders, and indicators of well-being, but no classic life history traits and none of the behavioral traits that are usually regarded as core life history markers in humans (e.g., impulsivity, risk-taking, sociosexuality). Thus, I am not suggesting that a different analysis of this particular matrix would recover a meaningful fast-slow continuum of variation. Some authors have proposed a general factor of mental and physical health ("covitality") as a component of slow life histories, with the rationale that slow strategists can invest more in somatic maintenance
} 
along an axis of individual "quality" does not falsify the hypothesis of a fast-slow continuum; in fact, the functional relations generated by life history trade-offs may become apparent only after the general quality factor has been statistically controlled for (see e.g., McLean et al., 2019; Wilson, 2014). More precisely, this is the expectation if the analysis is restricted to classic life history traits such as fertility and longevity. As I discuss next, behavioral and physiological traits often show complex functional relations that may not be adequately represented by linear correlations. Also, the role of individual condition/resources may be complicated by the presence of bidirectional relations with life history allocations (Figure 3; Roff \& Fairbairn, 2007). On the other hand, the behavioral and physiological traits that mediate life history allocations can be more revealing of the underlying trade-offs than the outcomes of those allocations. For example, the desire for short-term sexual encounters and that for stable, long-term relationships play a role in mediating the trade-off between mating and parenting at the behavioral level; these dispositions are negatively correlated (e.g., Holtzman \& Strube, 2013; Jackson \& Kirkpatrick, 2007), even if higher-quality (e.g., richer, healthier, more attractive) individuals have more access to short-term sexual partners and can provide better investment for their children.

3.4.3. Complex relations among traits. A common pitfall of the fast-slow paradigm is the (often implicit) assumption that relations between life history variables and their behavioral/physiological correlates should follow a simple linear pattern, so that fast versus slow strategies correspond to high versus low levels of the trait. For example, Réale et al. (2010) predicted - among other things - that "slow" individuals would tend to be nonaggressive, shy, and high in hypothalamic-pituitary-adrenal (HPA) reactivity, whereas "fast" individuals would be aggressive, bold, and low in HPA reactivity. Similar predictions in the domain of cognition were made by Sih and Del Giudice (2012). While this kind of pattern may apply to some traits with a relatively straightforward role in allocation trade-offs (e.g., impulsivity), as a general expectation it can be seriously misleading. Physiology and behavior often show complex and context-dependent relations, both with one another and with life history-relevant outcomes (e.g., Adkins-Regan, 2005; Salzman et al., 2018). Even if a trait is functionally related to the fast-slow axis of variation, the form of the relation can easily be nonlinear and/or interactive.

For an illustration, consider the adaptive calibration model of stress responsivity in humans (Del Giudice et al., 2011; Ellis \& Del Giudice, 2014). A key prediction of the model is that the optimal level of HPA reactivity should vary nonlinearly as one moves from extremely safe and supportive to extremely dangerous environments, so that both "fast" and "slow" constellations of traits can be associated with high (or low) HPA reactivity. In addition, we predicted an interaction with sex, with males switching to low-reactivity patterns more readily than females when growing up in dangerous, high-risk environments. If the model is broadly correct (see Ellis \& Del Giudice, 2019 for an empirical update), linear correlations between HPA reactivity and other life history-related traits will yield inconsistent findings, obscuring the role of the HPA axis in the regulation of life history strategies.

and are likely to receive better care from parents (Figueredo et al., 2004, 2007). However, this argument fails to consider the role of deleterious mutations. In later work, Sefcek \& Figueredo (2010) acknowledged that mutation load must be included in the picture, and that the relation between health and life history strategies is likely more complex than they originally envisioned. 
Nonlinear and interactive associations present obvious problems to analytic techniques based on linear associations, including standard varieties of PCA, factor analysis, and network analysis. Attempts to recover a fast-slow continuum by analyzing correlation matrices of behavioral and/or physiological traits are fraught with problems. This is unfortunate, but the relative simplicity of the fast-slow continuum does not automatically extend to the behavioral and physiological traits that mediate the underlying trade-offs. In some cases, the relations between traits and outcomes may be relatively straightforward and adequately described by simple linear models; but other times, the only solution is a detailed functional understanding of the trait and its costs, benefits, and constraints, which may vary across species and contexts (see Mathot \& Frankenhuis, 2018).

3.4.4. A note on measurement. In the human literature based on the fast-slow paradigm, behavioral traits are typically assessed via self-report. In recent years, some widely used questionnaires have been criticized for lacking external validity, employing circular or otherwise inadequate validation procedures, and mixing conceptually distinct types of constructs within a single measure (for an overview of the debate see Black et al., 2017; Copping et al., 2014b, 2017; Figueredo et al., 2015; Richardson et al., 2017b; Zietsch \& Sidari, 2020). To address the perceived limitations of self-reports, some researchers have started to measure putative life history-related traits such as impulsivity, risk-taking, and cooperation with standardized tasks instead of self-reports (for examples within the fast-slow paradigm, see Copping et al., 2014a; Wu et al., 2017). The available procedures include motor/attentional inhibition tasks, monetary choice tasks involving delays or variable outcomes, and economic games designed to elicit altruism, trust, and prosociality. These tasks lend themselves to computational analysis and promise to be more objective than traditional self-reports.

In practice, however, the validity of laboratory task turns out to be questionable. For example, executive tests of inhibition also measure general intelligence (Friedman et al., 2008), and delay discounting tasks tend to mistake risk aversion for impulsivity (Lopez-Guzman et al., 2018). As a result, self-reported impulsivity and disinhibition predict real-world outcomes (e.g., substance use, sexual behavior) much better than standardized tasks (Creswell et al., 2019; Leeman et al., 2019; Venables et al., 2018; Wilson \& Daly, 2006). Likewise, economic games in the laboratory often fail to predict similar behaviors in the field (e.g., Galizzi \& NavarroMartinez, 2018; Voors et al., 2012). Of course, games are very brief samples of behavior, and their individual reliability is low; for example, the correlations of economic games with other measures of cooperation and prosociality are usually smaller than .20, which helps explain the inconsistent results in the literature (e.g., Ferguson et al., 2019; McAuliffe et al., 2019; Zhao \& Smillie, 2015; see also Dang et al., 2020). The standardized behavioral assays used to assess personality in nonhuman animals suffer from similar problems (e.g., Beckmann \& Biro, 2013; Carter et al., 2012; Niemelä \& Dingemanse, 2018), but without the option of using self-reports. While laboratory tasks can be informative, they are far from "gold standards" and should be used and interpreted with caution.

3.4.5. Trade-offs and life history-related traits in humans. As I noted earlier, the general concept of the fast-slow continuum should be adapted to the particular ecology of each species to work as a useful heuristic. In the supplementary material (S3) I offer some reflections on certain notable features of the human ecology, and their implications for life history trade-offs 
and strategies. I also summarize my recent proposal for a descriptive model of life history-related traits in our species (Del Giudice, 2018).

\section{Conclusion}

Over the past thirty years, the fast-slow paradigm of individual differences has stimulated a remarkable amount of research. At the same time, empirical work in this area has too often drifted away from its theoretical premises, and many important gaps and questions have remained unaddressed. Now the paradigm is entering a new life stage - perhaps moving from a turbulent adolescence to the beginnings of maturity. In this paper I made a systematic attempt to lay out the logic of the paradigm, point out its current weaknesses, and identify opportunities for progress and improvement. I have argued that the fast-slow continuum can be a productive heuristic for individual differences, but there is clearly much work to do. I look forward to a new wave of research in this area, and to the insights and surprises it will certainly bring.

\section{Acknowledgments}

Heartfelt thanks to all who provided feedback and comments on this manuscript: Candace Black, Eric Charnov, Willem Frankenhuis, Steve Gangestad, JD Haltigan, Severi Luoto, Joe Manson, George Richardson, Oliver Sng, Jon Wright, Brendan Zietsch, and two anonymous reviewers.

\section{References}

Adkins-Regan, E. (2005). Hormones and animal social behavior. Princeton, NJ: Princeton University Press.

Alvergne, A., Jokela, M., \& Lummaa, V. (2010). Personality and reproductive success in a highfertility human population. Proceedings of the National Academy of Sciences USA, 107, $11745-11750$.

André, J. B., \& Rousset, F. (2020). Does extrinsic mortality accelerate the pace of life? A barebones approach. Evolution and Human Behavior. Doi:

10.1016/j.evolhumbehav.2020.03.002

Araya-Ajoy, Y. G., Bolstad, G. H., Brommer, J., Careau, V., Dingemanse, N. J., \& Wright, J. (2018). Demographic measures of an individual's "pace of life": Fecundity rate, lifespan, generation time, or a composite variable? Behavioral Ecology and Sociobiology, 72, 75.

Bakewell, A. T., Davis, K. E., Freckleton, R. P., Isaac, N. J., \& Mayhew, P. J. (2020). Comparing life histories across taxonomic groups in multiple dimensions: How mammallike are insects? The American Naturalist, 195, 70-81.

Baldini, R. (2015). Harsh environments and "fast" human life histories: What does the theory say? BioRxiv, 014647.

Barbaro, N., Boutwell, B. B., Barnes, J. C., \& Shackelford, T. K. (2017). Genetic confounding of the relationship between father absence and age at menarche. Evolution and Human Behavior, 38, 357-365. 
Bartels, M., Van den Berg, M., Sluyter, F., Boomsma, D. I., \& de Geus, E. J. (2003). Heritability of cortisol levels: Review and simultaneous analysis of twin studies.

Psychoneuroendocrinology, 28, 121-137.

Bassar, R. D., López-Sepulcre, A., Walsh, M. R., Turcotte, M. M., Torres-Mejia, M., \& Reznick, D. N. (2010). Bridging the gap between ecology and evolution: Integrating density regulation and life-history evolution. Annals of the New York Academy of Sciences, 1206, $17-34$.

Bateson, P., Gluckman, P., \& Hanson, M. (2014). The biology of developmental plasticity and the Predictive Adaptive Response hypothesis. Journal of Physiology, 592, 2357-2368.

Beckmann, C., \& Biro, P. A. (2013). On the validity of a single (boldness) assay in personality research. Ethology, 119, 937-947.

Belsky, J., \& Pluess, M. (2013). Beyond risk, resilience, and dysregulation: Phenotypic plasticity and human development. Development and Psychopathology, 25, 1243-1261.

Belsky, J., Steinberg, L., \& Draper, P. (1991). Childhood experience, interpersonal development, and reproductive strategy: An evolutionary theory of socialization. Child Development, 62, 647-670.

Berrigan, D., \& Koella, J. C. (1994). The evolution of reaction norms: Simple models for age and size at maturity. Journal of Evolutionary Biology, 7, 549-566.

Bertram, J., \& Masel, J. (2019). Different mechanisms drive the maintenance of polymorphism at loci subject to strong versus weak fluctuating selection. Evolution, 73, 883-896.

Bielby, J., Mace, G. M., Bininda-Emonds, O. R., Cardillo, M., Gittleman, J. L., Jones, K. E., ... \& Purvis, A. (2007). The fast-slow continuum in mammalian life history: An empirical reevaluation. The American Naturalist, 169, 748-757.

Bitarello, B. D., de Filippo, C., Teixeira, J. C., Schmidt, J. M., Kleinert, P., Meyer, D., \& Andrés, A. M. (2018). Signatures of long-term balancing selection in human genomes. Genome Biology and Evolution, 10, 939-955.

Black, C. J., Figueredo, A. J., \& Jacobs, W. J. (2017). Substance, history, and politics: An examination of the conceptual underpinnings of alternative approaches to the life history narrative. Evolutionary Psychology, 15, 1474704916670402.

Boyce, A. J., Mouton, J. C., Lloyd, P., Wolf, B. O., \& Martin, T. E. (2020). Metabolic rate is negatively linked to adult survival but does not explain latitudinal differences in songbirds. Ecology Letters, doi: 10.1111/ele.13464

Braendle, C., Heyland, F., \& Flatt, T. (2011). Integrating mechanistic and evolutionary analysis of life history variation. In T. Flatt \& F. Heyland (Eds.), Mechanisms of life history evolution. The genetics and physiology of life history traits and trade-offs (pp. 3-10). New York: Oxford University Press.

Briley, D. A., Tropf, F. C., \& Mills, M. C. (2017). What explains the heritability of completed fertility? Evidence from two large twin studies. Behavior Genetics, 47, 36-51.

Briley, D. A., \& Tucker-Drob, E. M. (2017). Comparing the developmental genetics of cognition and personality over the life span. Journal of Personality, 85, 51-64.

Brown, J. H., Gillooly, J. F., Allen, A. P., Savage, V. M., \& West, G. B. (2004). Toward a metabolic theory of ecology. Ecology, 85, 1771-1789. 
Brown, J. H., \& Sibly, R. M. (2006). Life-history evolution under a production constraint. Proceedings of the National Academy of Sciences USA, 103, 17595-17599.

Cam, E., Aubry, L. M., \& Authier, M. (2016). The conundrum of heterogeneities in life history studies. Trends in Ecology \& Evolution, 31, 872-886.

Carter, A. J., Marshall, H. H., Heinsohn, R., \& Cowlishaw, G. (2012). How not to measure boldness: novel object and antipredator responses are not the same in wild baboons. Animal Behaviour, 84, 603-609.

Caswell, H. (2007). Extrinsic mortality and the evolution of senescence. Trends in Ecology \& Evolution, 4, 173-174.

Caswell, H. (2009) Stage, age and individual stochasticity in demography. Oikos, 118, 17631782.

Chabris, C. F., Lee, J. J., Cesarini, D., Benjamin, D. J., \& Laibson, D. I. (2015). The fourth law of behavior genetics. Current Directions in Psychological Science, 24, 304-312.

Chang, L., \& Lu, H. J. (2018). Resource and extrinsic risk in defining fast life histories of rural Chinese left-behind children. Evolution and Human Behavior, 39, 59-66.

Chang, L., Lu, H. J., Lansford, J. E., Bornstein, M. H., Steinberg, L., Chen, B. B., ... \& Pastorelli, C. (2019a). External environment and internal state in relation to life-history behavioural profiles of adolescents in nine countries. Proceedings of the Royal Society of London B, 286, 20192097.

Chang, L., Lu, H. J., Lansford, J. E., Skinner, A. T., Bornstein, M. H., Steinberg, L., ... \& DeaterDeckard, K. (2019b). Environmental harshness and unpredictability, life history, and social and academic behavior of adolescents in nine countries. Developmental Psychology, 55, 890-903.

Charlesworth, B. (1994). Evolution in age-structured populations (2 $2^{\text {nd }}$ ed.). New York: Cambridge University Press.

Charnov, E. L. (1991). Evolution of life history variation among female mammals. Proceedings of the National Academy of Sciences USA, 88, 1134-1137.

Charnov, E. L., \& Berrigan, D. (1993). Why do female primates have such long lifespans and so few babies? Or life in the slow lane. Evolutionary Anthropology, 1, 191-194.

Charnov, E. L., Gislason, H., \& Pope, J. G. (2013). Evolutionary assembly rules for fish life histories. Fish and Fisheries, 14, 213-224.

Chippindale, A. K., Ngo, A. L., \& Rose, M. R. (2003). The devil in the details of life-history evolution: Instability and reversal of genetic correlations during selection on Drosophila development. Journal of Genetics, 82, 133.

Chisholm, J. S. (1993). Death, hope, and sex: Life-history theory and the development of reproductive strategies. Current Anthropology, 34, 1-24.

Chisholm, J. S. (1999). Death, hope, and sex: Steps to an evolutionary ecology of mind and morality. Cambridge: Cambridge University Press.

Connallon, T., \& Clark, A. G. (2013). Antagonistic versus nonantagonistic models of balancing selection: Characterizing the relative timescales and hitchhiking effects of partial selective sweeps. Evolution, 67, 908-917. 
Conner, J. K., Karoly, K., Stewart, C., Koelling, V. A., Sahli, H. F., \& Shaw, F. H. (2011). Rapid independent trait evolution despite a strong pleiotropic genetic correlation. The American Naturalist, 178, 429-441.

Copping, L. T., Campbell, A., \& Muncer, S. (2014a). Conceptualizing time preference: A lifehistory analysis. Evolutionary Psychology, 12, 829-847.

Copping, L. T., Campbell, A., \& Muncer, S. (2014b). Psychometrics and life history strategy: The structure and validity of the high K strategy scale. Evolutionary Psychology, 12, $200-222$.

Copping, L. T., Campbell, A., Muncer, S., \& Richardson, G. B. (2017). The psychometric evaluation of human life histories: A reply to Figueredo, Cabeza de Baca, Black, Garcia, Fernandes, Wolf, and Woodley (2015). Evolutionary Psychology, 1474704916663727.

Cox, R. M., McGlothlin, J. W., \& Bonier, F. (2016). Hormones as mediators of phenotypic and genetic integration: An evolutionary genetics approach. Integrative and Comparative Biology, 56, 126-137.

Crespi, B. (2014). An evolutionary framework for psychological maladaptations. Psychological Inquiry, 25, 322-324.

Crespi, E. J., Williams, T. D., Jessop, T. S., \& Delehanty, B. (2013). Life history and the ecology of stress: How do glucocorticoid hormones influence life-history variation in animals? Functional Ecology, 27, 93-106.

Creswell, K. G., Wright, A. G., Flory, J. D., Skrzynski, C. J., \& Manuck, S. B. (2019). Multidimensional assessment of impulsivity-related measures in relation to externalizing behaviors. Psychological Medicine, 49, 1678-1690.

Dammhahn, M., Dingemanse, N. J., Niemelä, P. T., \& Réale, D. (2018). Pace-of-life syndromes: a framework for the adaptive integration of behaviour, physiology and life history. Behavioral Ecology and Sociobiology, 72, 62.

Dang, J., King, K. M., \& Inzlicht, M. (2020). Why are self-report and behavioral measures weakly correlated? Trends in Cognitive Sciences, 24, 267-269.

Dańko, M. J., Burger, O., Argasiński, K., \& Kozłowski, J. (2018). Extrinsic mortality can shape life-history traits, including senescence. Evolutionary Biology, 45, 395-404.

Dańko, M. J., Burger, O., \& Kozłowski, J. (2017). Density-dependence interacts with extrinsic mortality in shaping life histories. PLoS ONE, 12, e0186661.

Dantzer, B., \& Swanson, E. M. (2017). Does hormonal pleiotropy shape the evolution of performance and life history traits? Integrative and Comparative Biology, 57, 372-384.

Deater-Deckard, K., Li, M., Lee, J., King-Casas, B., \& Kim-Spoon, J. (2019). Poverty and puberty: A neurocognitive study of inhibitory control in the transition to adolescence. Psychological Science, 30, 1573-1583.

Del Giudice, M. (2012). Sex ratio dynamics and fluctuating selection on personality. Journal of Theoretical Biology, 297, 48-60.

Del Giudice, M. (2014a). Life history plasticity in humans: The predictive value of early cues depends on the temporal structure of the environment. Proceedings of the Royal Society of London B, 281, 20132222.

Del Giudice, M. (2014b). A tower unto Heaven: Toward an expanded framework for psychopathology. Psychological Inquiry, 25, 394-413. 
Del Giudice, M. (2016). Differential susceptibility to the environment: Are developmental models compatible with the evidence from twin studies? Developmental Psychology, 52, 1330-1339.

Del Giudice, M. (2018). Evolutionary psychopathology: A unified approach. New York: Oxford University Press.

Del Giudice, M., Ellis, B. J., \& Shirtcliff, E. A. (2011). The Adaptive Calibration Model of stress responsivity. Neuroscience \& Biobehavioral Reviews, 35, 1562-1592.

Del Giudice, M., Gangestad, S. W., \& Kaplan, H. S. (2015). Life history theory and evolutionary psychology. In D. M. Buss (Ed.), The handbook of evolutionary psychology - Vol 1: Foundations (2 ${ }^{\text {nd }}$ ed.) (pp. 88-114). New York: Wiley.

Dingemanse, N. J., \& Dochtermann, N. A. (2013). Quantifying individual variation in behaviour: Mixed-effect modelling approaches. Journal of Animal Ecology, 82, 39-54.

Dobson, F. S., \& Oli, M. K. (2007). Fast and slow life histories of mammals. Ecoscience, 14, 292-299.

Dochtermann, N. A. (2011). Testing Cheverud's conjecture for behavioral correlations and behavioral syndromes. Evolution, 65, 1814-1820.

Dochtermann, N. A., Schwab, T., \& Sih, A. (2015). The contribution of additive genetic variation to personality variation: Heritability of personality. Proceedings of the Royal Society of London B, 282, 20142201.

Draghi, J. A., \& Whitlock, M. C. (2012). Phenotypic plasticity facilitates mutational variance, genetic variance, and evolvability along the major axis of environmental variation. Evolution, 66, 2891-2902.

Dunn, J., Andrews, C., Nettle, D., \& Bateson, M. (2019). Developmental history, energetic state and choice impulsivity in European starlings, Sturnus vulgaris. Animal Cognition, 22, 413-421.

Ellis, B. J. (2013). The hypothalamic-pituitary-gonadal axis: A switch-controlled, conditionsensitive system in the regulation of life history strategies. Hormones and Behavior, 64, 215-225.

Ellis, B. J., Boyce, W. T., Belsky, J., Bakermans-Kranenburg, M. J., \& van IJzendoorn, M. H. (2011). Differential susceptibility to the environment: An evolutionaryneurodevelopmental theory. Development and Psychopathology, 23, 7-28.

Ellis, B. J., \& Del Giudice, M. (2014). Beyond allostatic load: Rethinking the role of stress in regulating human development. Development and Psychopathology, 26, 1-20.

Ellis, B. J., \& Del Giudice, M. (2019). Developmental adaptation to stress: An evolutionary perspective. Annual Review of Psychology, 70, 111-139.

Ellis, B. J., Figueredo, A. J., Brumbach, B. H., \& Schlomer, G. L. (2009). The impact of harsh versus unpredictable environments on the evolution and development of life history strategies. Human Nature, 20, 204-268.

Ellner, S., \& Sasaki, A. (1996). Patterns of genetic polymorphism maintained by fluctuating selection with overlapping generations. Theoretical Population Biology, 50, 31-65.

Engen, S., Lande, R., \& Sæther, B. E. (2013). A quantitative genetic model of $r$-and $K$-selection in a fluctuating population. The American Naturalist, 181, 725-736. 
Engen, S., \& Sæther, B. E. (2016). Optimal age of maturity in fluctuating environments under $r$ and $K$-selection. Oikos, $125,1577-1585$.

Engen, S., \& Sæther, B. E. (2017). $r$-and $K$-selection in fluctuating populations is determined by the evolutionary trade-off between two fitness measures: Growth rate and lifetime reproductive success. Evolution, 71, 167-173.

Fawcett, T. W., Hamblin, S., \& Giraldeau, L. A. (2013). Exposing the behavioral gambit: The evolution of learning and decision rules. Behavioral Ecology, 24, 2-11.

Fenneman, J., \& Frankenhuis, W. E. (in press). Is impulsive behavior adaptive in harsh and unpredictable environments? A formal model. Evolution and Human Behavior. https://doi.org/10.1016/j.evolhumbehav.2020.02.005

Ferguson, E., Zhao, K., O'Carroll, R. E., \& Smillie, L. D. (2019). Costless and costly prosociality: Correspondence among personality traits, economic preferences, and realworld prosociality. Social Psychological and Personality Science, 10, 461-471.

Figueredo, A. J., de Baca, T. C., Black, C. J., García, R. A., Fernandes, H. B. F., Wolf, P. S. A., \& Woodley of Menie, M. A. (2015). Methodologically sound: Evaluating the psychometric approach to the assessment of human life history. Evolutionary Psychology, 13, 147470491501300202.

Figueredo, A. J., Vásquez, G., Brumbach, B. H., \& Schneider, S. M. R. (2004). The heritability of life history strategy: The k-factor, covitality, and personality. Social Biology, 51, 121143.

Figueredo, A. J., Vasquez, G., Brumbach, B. H., \& Schneider, S. M. R. (2007). The K-factor, covitality, and personality: A psychometric test of life history theory. Human Nature, 18 , 47-73.

Figueredo, A. J., Vásquez, G., Brumbach, B. H., Schneider, S. M. R., Sefcek, J. A., Tal, I. R., et al. (2006). Consilience and Life History Theory: From genes to brain to reproductive strategy. Developmental Review, 26, 243-275.

Fijarczyk, A., \& Babik, W. (2015). Detecting balancing selection in genomes: Limits and prospects. Molecular Ecology, 24, 3529-3545.

Frankenhuis, W. E., \& Del Giudice, M. (2012). When do adaptive developmental mechanisms yield maladaptive outcomes? Developmental Psychology, 48, 628-642.

Frankenhuis, W. E., Nettle, D., \& Dall, S. R. (2019). A case for environmental statistics of earlylife effects. Philosophical Transactions of the Royal Society of London B, 374, 20180110.

Franz, C. E., York, T. P., Eaves, L. J., Mendoza, S. P., Hauger, R. L., Hellhammer, D. H., ... \& Prom-Wormley, E. (2010). Genetic and environmental influences on cortisol regulation across days and contexts in middle-aged men. Behavior Genetics, 40, 467-479.

Friedman, N. P., Miyake, A., Young, S. E., DeFries, J. C., Corley, R. P., \& Hewitt, J. K. (2008). Individual differences in executive functions are almost entirely genetic in origin. Journal of Experimental Psychology: General, 137, 201-225.

Galizzi, M. M., \& Navarro-Martínez, D. (2018). On the external validity of social preference games: A systematic lab-field study. Management Science, 65, 976-1002.

Gangestad, S. W. (2011). Evolutionary processes explaining the genetic variance in personality: An exploration of scenarios. In D. M. Buss \& P. H. Hawley (Eds.), The evolution of 
personality and individual differences (pp. 338-375). New York: Oxford University Press.

Gerish, B., \& Antebi, A. (2011). Molecular basis of life history regulation in C. elegans and other organisms. In T. Flatt \& F. Heyland (Eds.), Mechanisms of life history evolution: The genetics and physiology of life history traits and trade-offs (pp. 284-298). New York: Oxford University Press.

Grafen, A. (1984). Natural selection, kin selection and group selection. In J. R. Krebs \& N. B. Davies (Eds.), Behavioural Ecology: An Evolutionary Approach (pp. 62-84). Oxford, UK: Blackwell.

Gurven, M., von Rueden, C., Stieglitz, J., Kaplan, H., \& Rodriguez, D. E. (2014). The evolutionary fitness of personality traits in a small-scale subsistence society. Evolution and Human Behavior, 35, 17-25.

Hartemink, N., \& Caswell, H. (2018). Variance in animal longevity: Contributions of heterogeneity and stochasticity. Population Ecology, 60, 89-99.

Hau, M., Casagrande, S., Ouyang, J. Q., \& Baugh, A. T. (2016). Glucocorticoid-mediated phenotypes in vertebrates: Multilevel variation and evolution. Advances in the Study of Behavior, 48, 41-115.

Hau, M., \& Wingfield, J. C. (2011). Hormonally regulated trade-offs: Evolutionary variability and phenotypic plasticity in testosterone signaling pathways. In T. Flatt \& F. Heyland (Eds.), Mechanisms of life history evolution. The genetics and physiology of life history traits and trade-offs (pp. 349-362). New York, NY: Oxford University Press.

Healy, K., Ezard, T. H., Jones, O. R., Salguero-Gómez, R., \& Buckley, Y. M. (2019). Animal life history is shaped by the pace of life and the distribution of age-specific mortality and reproduction. Nature Ecology \& Evolution, 3, 1217-1224.

Hendry, A. P. (2015). Key questions on the role of phenotypic plasticity in eco-evolutionary dynamics. Journal of Heredity, 107, 25-41.

Hill, W. D., Arslan, R. C., Xia, C., Luciano, M., Amador, C., Navarro, P., ... \& Deary, I. J. (2018). Genomic analysis of family data reveals additional genetic effects on intelligence and personality. Molecular Psychiatry, 23, 2347-2362.

Holdsworth, E. A., \& Appleton, A. A. (2020). Adverse childhood experiences and reproductive strategies in a contemporary US population. American Journal of Physical Anthropology, 171, 37-49.

Holtzman, N. S., \& Strube, M. J. (2013). Above and beyond short-term mating, long-term mating is uniquely tied to human personality. Evolutionary Psychology, 11, 147470491301100514.

Houle, D. (1991). Genetic covariance of fitness correlates: What genetic correlations are made of and why it matters. Evolution, 45, 630-648.

Ibitoye, M., Choi, C., Tai, H., Lee, G., \& Sommer, M. (2017). Early menarche: A systematic review of its effect on sexual and reproductive health in low-and middle-income countries. PLoS ONE, 12(, e0178884.

Jackson, J. J., \& Kirkpatrick, L. A. (2007). The structure and measurement of human mating strategies: Toward a multidimensional model of sociosexuality. Evolution and Human Behavior, 28, 382-391. 
Jeschke, J. M., Gabriel, W., \& Kokko, H. (2008). r-Strategist/K-Strategists. In S. E. Jørgensen \& B. D. Fath (Eds.), Encyclopedia of ecology (pp. 3113-3122). Oxford: Elsevier.

Jeschke, J. M., \& Kokko, H. (2009). The roles of body size and phylogeny in fast and slow life histories. Evolutionary Ecology, 23, 867-878.

Jokela, M., Alvergne, A., Pollet, T. V., \& Lummaa, V. (2011). Reproductive behavior and personality traits of the Five Factor Model. European Journal of Personality, 25, 487500.

Kawecki, T. J., \& Stearns, S. C. (1993). The evolution of life histories in spatially heterogeneous environments: optimal reaction norms revisited. Evolutionary Ecology, 7, 155-174.

Keller, M. C. (2018). Evolutionary perspectives on genetic and environmental risk factors for psychiatric disorders. Annual Review of Clinical Psychology, 14, 471-493.

Kendler, K. S., Ohlsson, H., Lichtenstein, P., Sundquist, J., \& Sundquist, K. (2019). The nature of the shared environment. Behavior Genetics, 49, 1-10.

Ketterson, E. D., \& Nolan, Jr, V. (1999). Adaptation, exaptation, and constraint: A hormonal perspective. The American Naturalist, 154, S4-S25.

Ketterson, E. D., Atwell, J. W., \& McGlothlin, J. W. (2009). Phenotypic integration and independence: hormones, performance, and response to environmental change. Integrative and Comparative Biology, 49, 365-379.

Kosova, G., Abney, M., \& Ober, C. (2010). Heritability of reproductive fitness traits in a human population. Proceedings of the National Academy of Sciences USA, 107, 1772-1778.

Kruuk, L. E., Slate, J., \& Wilson, A. J. (2008). New answers for old questions: The evolutionary quantitative genetics of wild animal populations. Annual Review of Ecology, Evolution, and Systematics, 39, 525-548.

Lancaster, L. T., \& Sinervo, B. (2011). Epistatic social and endocrine networks and the evolution of life history trade-offs and plasticity. In T. Flatt \& F. Heyland (Eds.), Mechanisms of life history evolution. The genetics and physiology of life history traits and trade-offs (pp. 329-348). New York: Oxford University Press.

Lande, R., Engen, S., \& Sæther, B. E. (2009). An evolutionary maximum principle for densitydependent population dynamics in a fluctuating environment. Philosophical Transactions of the Royal Society of London B, 364, 1511-1518.

Lande, R., Engen, S., \& Sæther, B. E. (2017). Evolution of stochastic demography with life history tradeoffs in density-dependent age-structured populations. Proceedings of the National Academy of Sciences USA, 114, 11582-11590.

Leeman, R. F., Rowland, B. H., Gebru, N. M., \& Potenza, M. N. (2018). Relationships among impulsive, addictive and sexual tendencies and behaviours: A systematic review of experimental and prospective studies in humans. Philosophical Transactions of the Royal Society of London B, 374, 20180129.

Lian, Q., Zuo, X., Mao, Y., Zhang, Y., Luo, S., Zhang, S., ... \& Zhou, W. (2018). The impact of the Wenchuan earthquake on early puberty: A natural experiment. PeerJ, 6, e5085.

Lopez-Guzman, S., Konova, A. B., Louie, K., \& Glimcher, P. W. (2018). Risk preferences impose a hidden distortion on measures of choice impulsivity. PLOS ONE, 13, e0191357.

MacArthur, R. H., \& Wilson, E. O. (1967). The theory of island biogeography. Princeton, NJ: Princeton University Press. 
Macaskill, A. C., Hunt, M. J., \& Milfont, T. L. (2019). On the associations between delay discounting and temporal thinking. Personality and Individual Differences, 141, 166-172.

Madrigal, L., Relethford, J. H., \& Crawford, M. H. (2003). Heritability and anthropometric influences on human fertility. American Journal of Human Biology, 15, 16-22.

Malerba, M. E., \& Marshall, D. J. (2019). Size-abundance rules? Evolution changes scaling relationships between size, metabolism and demography. Ecology Letters, 22, 1407-1416.

Mathot, K. J., \& Frankenhuis, W. E. (2018). Models of pace-of-life syndromes (POLS): A systematic review. Behavioral Ecology and Sociobiology, 72, 41.

McAuliffe, W. H., Forster, D. E., Pedersen, E. J., \& McCullough, M. E. (2019). Does cooperation in the laboratory reflect the operation of a broad trait? European Journal of Personality, 33, 89-103.

McLean, E. M., Archie, E. A., \& Alberts, S. C. (2019). Lifetime fitness in wild female baboons: Trade-offs and individual heterogeneity in quality. The American Naturalist, 194, 745 759.

McNamara, J. M., Dall, S. R., Hammerstein, P., \& Leimar, O. (2016). Detection vs. selection: Integration of genetic, epigenetic and environmental cues in fluctuating environments. Ecology Letters, 19, 1267-1276.

Međedović, J., Šoljaga, M., Stojković, A., \& Gojević, I. (2018). Revealing complex relations between personality and fitness: HEXACO personality traits, life-time reproductive success and the age at first birth. Personality and Individual Differences, 129, 143-148.

Mell, H., Safra, L., Algan, Y., Baumard, N., \& Chevallier, C. (2018). Childhood environmental harshness predicts coordinated health and reproductive strategies: A cross-sectional study of a nationally representative sample from France. Evolution and Human Behavior, 39, 18.

Messer, P. W., Ellner, S. P., \& Hairston Jr, N. G. (2016). Can population genetics adapt to rapid evolution? Trends in Genetics, 32, 408-418.

Morris, D. H., Jones, M. E., Schoemaker, M. J., Ashworth, A., \& Swerdlow, A. J. (2011). Familial concordance for age at menarche: Analyses from the Breakthrough Generations Study. Paediatric and Perinatal Epidemiology, 25, 306-311.

Mylius, S. D., \& Diekmann, O. (1995). On evolutionarily stable life histories, optimization and the need to be specific about density dependence. Oikos, 74, 218-224.

Nettle, D. (2011). Flexibility in reproductive timing in human females: Integrating ultimate and proximate explanations. Philosophical Transactions of the Royal Society of London B, $366,357-365$.

Nettle, D. (2018). Is it explanation yet? https://www.danielnettle.org.uk/2018/04/17/hotte-13-isit-explanation-yet/

Nettle, D., \& Bateson, M. (2015). Adaptive developmental plasticity: what is it, how can we recognize it and when can it evolve? Proceedings of the Royal Society of London B, 282, 20151005.

Nettle, D., \& Frankenhuis, W. E. (2019). The evolution of life-history theory: A bibliometric analysis of an interdisciplinary research area. Proceedings of the Royal Society of London $B, 286,20190040$. 
Nettle, D., Frankenhuis, W. E., \& Rickard, I. J. (2013). The evolution of predictive adaptive responses in human life history. Proceedings of the Royal Society of London B, 280, 20131343.

Nettle, D., Gibson, M. A., Lawson, D. W., \& Sear, R. (2013). Human behavioral ecology: current research and future prospects. Behavioral Ecology, 24, 1031-1040.

Niemelä, P. T., \& Dingemanse, N. J. (2018). On the usage of single measurements in behavioural ecology research on individual differences. Animal Behaviour, 145, 99-105.

Nolte, I. M., Tropf, F. C., \& Snieder, H. (2019). Missing heritability of complex traits and diseases. $e L S$, doi: 10.1002/9780470015902.a0028223

Oli, M. K. (2004). The fast-slow continuum and mammalian life-history patterns: An empirical evaluation. Basic and Applied Ecology, 5, 449-463.

Ouellet-Morin, I., Dionne, G., Pérusse, D., Lupien, S. J., Arseneault, L., Barr, R. G., ... \& Boivin, M. (2009). Daytime cortisol secretion in 6-month-old twins: Genetic and environmental contributions as a function of early familial adversity. Biological Psychiatry, 65, 409416.

Pavličev, M., \& Cheverud, J. M. (2015). Constraints evolve: Context dependency of gene effects allows evolution of pleiotropy. Annual Review of Ecology, Evolution, and Systematics, 46, 413-434.

Pavličev, M., Cheverud, J. M., \& Wagner, G. P. (2011). Evolution of adaptive phenotypic variation patterns by direct selection for evolvability. Proceedings of the Royal Society B, 278, 1903-1912.

Peiman, K. S., \& Robinson, B. W. (2017). Comparative analyses of phenotypic trait covariation within and among populations. The American Naturalist, 190, 451-468.

Penke, L., \& Jokela, M. (2016). The evolutionary genetics of personality revisited. Current Opinion in Psychology, 7, 104-109.

Pesonen, A. K., Räikkönen, K., Heinonen, K., Kajantie, E., Forsén, T., \& Eriksson, J. G. (2008). Reproductive traits following a parent-child separation trauma during childhood: A natural experiment during World War II. American Journal of Human Biology, 20, 345 351.

Pianka, E. R. (1970). On r- and K-selection. The American Naturalist, 104, 592-597.

Pigliucci, M. (2005). Evolution of phenotypic plasticity: where are we going now? Trends in Ecology \& Evolution, 20, 481-486.

Polderman, T. J., Benyamin, B., de Leeuw, C. A., Sullivan, P. F., van Bochoven, A., Visscher, P. M., et al. (2015). Meta-analysis of the heritability of human traits based on fifty years of twin studies. Nature Genetics, 47, 702-709.

Pollet, T. V., Tybur, J. M., Frankenhuis, W. E., \& Rickard, I. J. (2014). What can cross-cultural correlations teach us about human nature? Human Nature, 25, 410-429.

Promislow, D. E., \& Harvey, P. H. (1990). Living fast and dying young: A comparative analysis of life-history variation among mammals. Journal of Zoology, 220, 417-437.

Quinlan, R. J. (2007). Human parental effort and environmental risk. Proceedings of the Royal Society of London B, 274, 121-125. 
Réale, D., Garant, D., Humphries, M. M., Bergeron, P., Careau, V., \& Montiglio, P. O. (2010). Personality and the emergence of the pace-of-life syndrome concept at the population level. Philosophical Transactions of the Royal Society of London B, 365, 4051-4063.

Revell, L. J. (2007). The G matrix under fluctuating correlational mutation and selection. Evolution, 61, 1857-1872.

Reznick, D., Bryant, M. J., \& Bashey, F. (2002). $r$-and $K$-selection revisited: The role of population regulation in life-history evolution. Ecology, 83, 1509-1520.

Reznick, D., Nunney, L., \& Tessier, A. (2000). Big houses, big cars, superfleas and the costs of reproduction. Trends in Ecology and Evolution, 15, 421-425.

Richardson, G. B., Chen, C. C., Dai, C. L., Brubaker, M. D., \& Nedelec, J. L. (2017a). The psychometrics of the Mini-K: Evidence from two college samples. Evolutionary Psychology, 15, 1474704916682034.

Richardson, G. B., Sanning, B. K., Lai, M. H. C., Copping, L. T., Hardesty, P. H., \& Kruger, D. J. (2017b). On the psychometric study of human life history strategies: State of the science and evidence of two independent dimensions. Evolutionary Psychology, 1474704916666840.

Rickard, I. J., Frankenhuis, W. E., \& Nettle, D. (2014). Why are childhood family factors associated with timing of maturation? A role for internal prediction. Perspectives on Psychological Science, 9, 3-15.

Robinson, W. S. (1950). Ecological correlations and the behavior of individuals. American Sociological Review, 15, 351-357.

Roff, D. A. (1996). The evolution of genetic correlations: An analysis of patterns. Evolution, 50, 1392-1403.

Roff, D. A. (2002). Life history evolution. Sunderland, MA: Sinauer.

Roff, D. A., \& Fairbairn, D. J. (2007). The evolution of trade-offs: Where are we? Journal of Evolutionary Biology, 20, 433-447.

Ross, C. (1988). The intrinsic rate of natural increase and reproductive effort in primates. Journal of Zoology, 214, 199-219.

Royauté, R., Berdal, M. A., Garrison, C. R., \& Dochtermann, N. A. (2018). Paceless life? A meta-analysis of the pace-of-life syndrome hypothesis. Behavioral Ecology and Sociobiology, 72, 64.

Rüger, N., Comita, L. S., Condit, R., Purves, D., Rosenbaum, B., Visser, M. D., ... \& Wirth, C. (2018). Beyond the fast-slow continuum: demographic dimensions structuring a tropical tree community. Ecology Letters, 21, 1075-1084.

Sæther B. E. (1987) The influence of body weight on the covariation between reproductive traits in European birds. Oikos, 48, 79-88.

Sæther, B. E., \& Engen, S. (2015). The concept of fitness in fluctuating environments. Trends in Ecology \& Evolution, 30, 273-281.

Salguero-Gómez, R. (2017). Applications of the fast-slow continuum and reproductive strategy framework of plant life histories. New Phytologist, 213, 1618-1624.

Salguero-Gómez, R., Jones, O. R., Jongejans, E., Blomberg, S. P., Hodgson, D. J., Mbeau-Ache, C., ... \& Buckley, Y. M. (2016). Fast-slow continuum and reproductive strategies 
structure plant life-history variation worldwide. Proceedings of the National Academy of Sciences USA, 113, 230-235.

Saltz, J. B., Hessel, F. C., \& Kelly, M. W. (2017). Trait correlations in the genomics era. Trends in Ecology \& Evolution, 32, 279-290.

Salzman, T. C., McLaughlin, A. L., Westneat, D. F., \& Crowley, P. H. (2018). Energetic tradeoffs and feedbacks between behavior and metabolism influence correlations between pace-of-life attributes. Behavioral Ecology and Sociobiology, 72, 54.

Schmitt, D. P. (2019). Where's the fitness? Facultative "life history" variations in personality and attachment differentially linked to reproductive success across ecological contexts. Talk at the $31^{\text {st }}$ Annual Meeting of the Human Behavior \& Evolution society. May $29^{\text {th }}$ June $1^{\text {st }}, 2019$, Boston, MA.

Sear, R., Sheppard, P., \& Coall, D. A. (2019). Cross-cultural evidence does not support universal acceleration of puberty in father-absent households. Philosophical Transactions of the Royal Society of London B, 374, 20180124.

Sefcek, J. A., \& Figueredo, A. J. (2010). A life-history model of human fitness indicators. Biodemography and Social Biology, 56, 42-66.

Sella, G., \& Barton, N. H. (2019). Thinking about the evolution of complex traits in the era of genome-wide association studies. Annual Review of Genomics and Human Genetics, doi: 10.1146/annurev-genom-083115-022316

Sibly, R. M., \& Brown, J. H. (2007). Effects of body size and lifestyle on evolution of mammal life histories. Proceedings of the National Academy of Sciences USA, 104, 17707-17712.

Sih, A., \& Del Giudice, M. (2012). Linking behavioural syndromes and cognition: a behavioural ecology perspective. Philosophical Transactions of the Royal Society of London B, 367, 2762-2772.

Smith, B. R., \& Blumstein, D. T. (2008). Fitness consequences of personality: A meta-analysis. Behavioral Ecology, 19, 448-455.

Sng, O., \& Ackerman, J. M. (2020). Too many people, women, men? The psychological effects of population density and sex ratio. Current Opinion in Psychology, 32, 38-40.

Sng, O., Neuberg, S. L., Varnum, M. E., \& Kenrick, D. T. (2017). The crowded life is a slow life: Population density and life history strategy. Journal of Personality and Social Psychology, 112, 736-754.

Snyder, R. E., \& Ellner, S. P. (2018). Pluck or luck: Does trait variation or chance drive variation in lifetime reproductive success? The American Naturalist, 191, E90-E107.

Sodini, S. M., Kemper, K. E., Wray, N. R., \& Trzaskowski, M. (2018). Comparison of genotypic and phenotypic correlations: Cheverud's conjecture in humans. Genetics, 209, 941-948.

Starrfelt, J., \& Kokko, H. (2013). Bet-hedging - a triple trade-off between means, variances and correlations. Biological Reviews, 87, 742-755.

Stearns, S. C. (1983). The influence of size and phylogeny on patterns of covariation among lifehistory traits in the mammals. Oikos, 41, 173-187.

Stearns, S. C. (1989). Trade-offs in life-history evolution. Functional Ecology, 3, 259-268.

Stearns, S. C. (1992). The evolution of life histories. New York, NY: Oxford University Press.

Stearns, S. C., Byars, S. G., Govindaraju, D. R., \& Ewbank, D. (2010). Measuring selection in contemporary human populations. Nature Reviews Genetics, 11, 611. 
Stearns, S. C., \& Rodrigues, A. M. M. (2020). On the use of "life history theory" in evolutionary psychology. Evolution and Human Behavior. Doi: 10.1016/j.evolhumbehav.2020.02.001

Steiner, U. K., \& Tuljapurkar, S. (2012). Neutral theory for life histories and individual variability in fitness components. Proceedings of the National Academy of Sciences USA, 109, 4684-4689.

Surbey, M. K. (2014). Addressing our inner salmon in an evolutionary framework for psychopathology. Psychological Inquiry, 25, 369-375.

Swanson, E. M., \& Dantzer, B. (2014). Insulin-like growth factor-1 is associated with life-history variation across Mammalia. Proceedings of the Royal Society of London B, 281, 20132458.

Szepsenwol, O., Griskevicius, V., Simpson, J. A., Young, E. S., Fleck, C., \& Jones, R. E. (2017). The effect of predictable early childhood environments on sociosexuality in early adulthood. Evolutionary Behavioral Sciences, 11, 131-145.

Terracciano, A., Löckenhoff, C. E., Zonderman, A. B., Ferrucci, L., \& Costa Jr., P. T. (2008). Personality predictors of longevity: Activity, emotional stability, and conscientiousness. Psychosomatic Medicine, 70, 621-627.

Thompson, J. N. (2013). Relentless evolution. Chicago, IL: Chicago University Press.

Tinkle, D. W., Wilbur, H. M., \& Tilley, S. G. (1970). Evolutionary strategies in lizard reproduction. Evolution, 24, 55-74.

Tropf, F. C., Barban, N., Mills, M. C., Snieder, H., \& Mandemakers, J. J. (2015). Genetic influence on age at first birth of female twins born in the UK, 1919-68. Population studies, 69, 129-145.

Tucker-Drob, E. M., Grotzinger, A. D., Briley, D. A., Engelhardt, L. E., Mann, F. D., Patterson, M., ... \& Harden, K. P. (2017). Genetic influences on hormonal markers of chronic hypothalamic-pituitary-adrenal function in human hair. Psychological Medicine, 47, 1389-1401.

Uller, T., Moczek, A. P., Watson, R. A., Brakefield, P. M., \& Laland, K. N. (2018). Developmental bias and evolution: A regulatory network perspective. Genetics, 209, 949966.

van Noordwijk, A. J., \& de Jong, G. (1986). Acquisition and allocation of resources: Their influence on variation in life history tactics. The American Naturalist, 128, 137-142.

Venables, N. C., Foell, J., Yancey, J. R., Kane, M. J., Engle, R. W., \& Patrick, C. J. (2018). Quantifying inhibitory control as externalizing proneness: A cross-domain model. Clinical Psychological Science, 6, 561-580.

Verweij, K. J., Yang, J., Lahti, J., Veijola, J., Hintsanen, M., Pulkki-Råback, L., ... \& Taanila, A. (2012). Maintenance of genetic variation in human personality: Testing evolutionary models by estimating heritability due to common causal variants and investigating the effect of distant inbreeding. Evolution, 66, 3238-3251.

Vitousek, M. N., \& Schoenle, L. A. (2019). Hormones and behavior: A life history perspective. In L. M. Welling \& T. K. Shackelford (Eds.), The Oxford handbook of evolutionary psychology and behavioral endocrinology. New York: Oxford University Press. 
Voors, M., Turley, T., Kontoleon, A., Bulte, E., \& List, J. A. (2012). Exploring whether behavior in context-free experiments is predictive of behavior in the field: Evidence from lab and field experiments in rural Sierra Leone. Economics Letters, 114, 308-311.

Vukasović, T., \& Bratko, D. (2015). Heritability of personality: A meta-analysis of behavior genetic studies. Psychological Bulletin, 141, 769-785.

Wainschtein, P., Jain, D. P., Yengo, L., Zheng, Z., Cupples, L. A., Shadyab, A. H., ... \& Kooperberg, C. (2019). Recovery of trait heritability from whole genome sequence data. bioRxiv, 588020.

Weitekamp, C. A., \& Keller, L. (2019). Genes and behavior. In D. J. Hosken \& N. Wedell (Eds.), Genes and behaviour: Beyond nature-nurture (pp. 93-109). Hoboken, NJ: Wiley.

West-Eberhard, M. J. (2003). Developmental plasticity and evolution. New York: Oxford University Press.

Wilson, A. J. (2014). Competition as a source of constraint on life history evolution in natural populations. Heredity, 112, 70-78.

Wilson, M., \& Daly, M. (2006). Are juvenile offenders extreme future discounters? Psychological Science, 17, 989-994.

Wolf, M., van Doorn, G. S., Leimar, O., \& Weissing, F. J. (2007). Life-history trade-offs favour the evolution of animal personalities. Nature, 447, 581-585.

Wright, J., Bolstad, G. H., Araya-Ajoy, Y. G., \& Dingemanse, N. J. (2019). Life-history evolution under fluctuating density-dependent selection and the adaptive alignment of pace-of-life syndromes. Biological Reviews, 94, 230-247.

Wu, J., Balliet, D., Tybur, J. M., Arai, S., Van Lange, P. A., \& Yamagishi, T. (2017). Life history strategy and human cooperation in economic games. Evolution and Human Behavior, 38, 496-505.

Yamamichi, M., \& Hoso, M. (2017). Roles of maternal effects in maintaining genetic variation: Maternal storage effect. Evolution, 71, 449-457.

Zhao, K., \& Smillie, L. D. (2015). The role of interpersonal traits in social decision making: Exploring sources of behavioral heterogeneity in economic games. Personality and Social Psychology Review, 19, 277-302.

Zietsch, B. P., \& Sidari, M. J. (2020). A critique of life history approaches to human trait covariation. Evolution and Human Behavior, doi: 10.1016/j.evolhumbehav.2019.05.007 


\title{
Rethinking the Fast-Slow Continuum of Individual Differences
}

\author{
Supplementary Material
}

Marco Del Giudice (marcodg@unm.edu)

\section{S1. The problem of axis rotation and its implications for the fast-slow continuum}

In this supplement I address the pervasive but often overlooked problem of axis rotation in principal component analysis (PCA). As I note in the main text (Section 2.1), a number of seemingly inconsistent findings in the comparative life history literature are explained by irrelevant differences in the orientation of the axes used to summarize the data. Here I focus on PCA because it is the technique of choice in comparative studies; however, similar issues may arise in standard applications of exploratory factor analysis (EFA).

In comparative research, PCA is commonly used to identify the major axes of life history variation across species. For simplicity, in the examples that follow I assume that the data reflect two biologically significant axes of variation (the "true" axes), one of which represents a fastslow continuum (Figure S1.1). This is a common pattern in the life histories of animals (e.g., Healy et al., 2019) and plants (e.g., Salguero-Gómez, 2017). In the unrotated PCA solution, the first component (i.e., the first axis of the transformed space) follows the direction of maximum variance in the data; the second component follows the direction of largest variance uncorrelated with the first; and so on. However, the space defined by these components is one possibility out of infinitely many, since any rotation of the axes around the origin yields a mathematically equivalent description of the data. The only thing that makes the unrotated PCA solution unique is that the axes are oriented so as to maximize the proportion of variance explained. There is no reason why the unrotated axes should represent the most biologically meaningful dimensions of variation, unless the latter also happen to explain the largest amount of variance in the data.

It follows that, if the main axis of variation in the data accounts for a large proportion of the total variance, it will be adequately captured by the first unrotated component (PC1; Figure $\mathrm{S} 1.1 \mathrm{a})$. The first component tends to be general, with sizable loadings (positive or negative) from most or even all the original variables. This is what happens with life history variables when body size is not controlled for: the first unrotated component accounts for most of the variance (about $70-80 \%$ in mammals), and in most cases it clearly represents a fast-slow axis of variation (for examples see Figures S1.2a, S1.3a, and S1.3c).

In their study of life histories in mammals, Bielby et al. (2007) did not report the unrotated solution, but used a standard rotation method (varimax) to automatically rotate the components. Rotated components no longer follow the direction of maximum variance but instead optimize some other criterion, usually with the goal of making the solution more readily interpretable. The problem with varimax and other standard rotations is that they seek a so-called "simple structure," whereby each component has a few large loadings for some variables and small (preferably zero) loadings for the remaining ones (see Browne, 2001; Sass \& Schmitt, 2010). As a result, common rotation algorithms such as varimax and oblimin are designed to break up general components and factors; as a rule, they are unable to recover a general fast-slow continuum even if it exists. The main exceptions are quartimax and quartimin rotations, which typically produce a first general factor but are considerably less popular (see Browne, 2001; Darton, 1980; Sass \& Schmitt, 2010). 

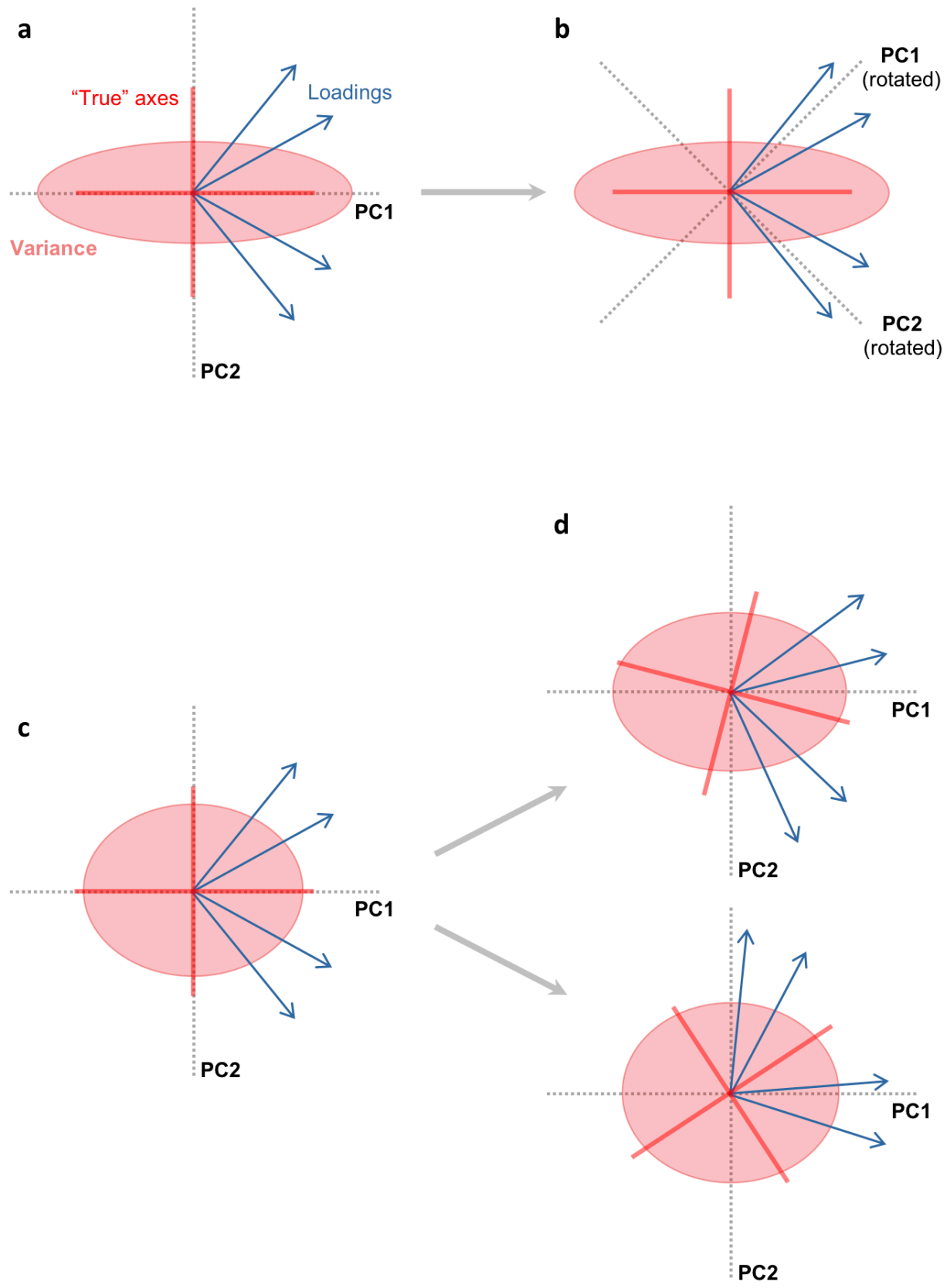

Figure S1.1. Schematic illustration of common rotation artifacts in PCA. In panel (a), the main axis of variation dominates the solution and aligns with the first unrotated component (PC1). Rotating the axes toward a simple structure (b) obscures the presence of a general dimension in the data; note that the rotated components are no longer aligned with the "true" axes. In panel (c), the two major axes explain similar proportions of variance in the data. The orientation of the axes becomes more sensitive to the composition of the sample, the specific variables included in the analysis, and so on (d). The two solutions shown in (d) are substantively equivalent, and differ only because of (irrelevant) changes in the orientation of the axes. Also note that the true axes of variation are not closely aligned with PC1 and PC2.

The effect of rotating the axes toward a simple structure is illustrated in Figure S1.1b. As has been noted before (e.g., Lykken, 1971), making the a priori assumption that the data will conform to a simple structure is not very reasonable when one is dealing with complex biological systems. And while this criterion may aid interpretation in other contexts, it is clearly inappropriate when the data are hypothesized to reflect a general dimension of variation. In 
Bielby et al. (2007), the varimax rotation returned two axes corresponding to offspring size versus number and timing of reproduction, but - predictably - no unitary fast-slow continuum. However, the unrotated solution based on the same data (shown in Figure S1.2c) is extremely similar to the one found by Stearns (1983; Figure S1.2a); in both cases, the first component describes a classic fast-slow continuum (for more details see Del Giudice, 2014). The coefficient of congruence of PC1 loadings for the variables included in both datasets is $>.99$ in the uncorrected solution and .86 in the mass-corrected solution (see Abdi, 2007).

Of course, the main axis of variation does not always dominate the solution as in Figure S1.1a. An alternative scenario (illustrated in Figure S1.1c) is one in which the two major axes explain a similar amount of variance. When this is the case, the orientation of the components becomes particularly sensitive to the composition of the sample, the variables included in the analysis, and the effects of measurement error. All these factors contribute to determining the direction of maximum variance, and hence the direction of the axes identified by PCA. As a consequence, solutions tend to become less stable, and the "true" axes of variation may end up in directions that do not align closely with any of the components (Figure S1.1d). This is especially likely to happen when life history data are corrected for body size: after correction, the first component becomes smaller while the second becomes proportionally larger, so that $\mathrm{PC} 1$ and PC2 often account for similar amounts of (residual) variance. To illustrate, the first and second component in mammals explain $70-80 \%$ and $10-15 \%$ of the variance in uncorrected data; but after correction for body size, they account for about $30-50 \%$ and $20-30 \%$ of the variance, respectively (see Del Giudice, 2014; Healy et al.,2019; Jeschke \& Kokko, 2009; Stearns, 1983). Thus, PCA solutions for mass-corrected data should often prove less stable than those for uncorrected data. It is also quite possible that the fast-slow continuum (if present) will not be neatly captured by either PC1 or PC2. Note that these problems may also occur with uncorrected data; but they become more likely when controlling for body size reduces the relative strength of the first component.

In the study by Jeschke and Kokko (2009), the uncorrected solution for mammals showed a clear fast-slow continuum on the first component (Figure S1.3a; the authors did not report loadings for PC2). In contrast, the first component of the mass-corrected solution looked rather different from the classic fast-slow continuum; this was interpreted as evidence that the uncorrected and mass-corrected continua are largely distinct constructs. However, Figure S1.3b tells a different story: the fast-slow continuum does not disappear in the mass-corrected solution - it simply aligns with PC2, rather than with PC1 as in the uncorrected solution (the coefficient of congruence between the uncorrected PC1 and the mass-corrected PC2 is .85). This "switch" between PC1 and PC2 is not surprising, since the two components account for very similar proportions of variance ( $37 \%$ and $29 \%)$.

Another interesting example of rotation artifact is shown in Figures S1.3e and S1.3f. Jeschke and Kokko (2009) compared the first component of the uncorrected and mass-corrected solutions in birds, found them to be markedly different, and interpreted this as further evidence that the nature of the fast-slow continuum changes after correction for body size. In fact, the two solutions are extremely similar, and only seem different because they are rotated in different directions (also, the sign of PC2 loadings is reversed in the mass-corrected solution-another irrelevant difference that contributes to masking the overwhelming similarity). For clarity, Figure S1.4 shows the same loading plots with two rotated axes superimposed on the original solutions. The axes were rotated so as to align with two key markers of the fast-slow continuum in birds and mammals, namely age at first reproduction and average fertility (labeled "fecundity" in the graph; more on this below). It is easy to see that - despite the superficial dissimilarity of the 
original solutions - the two rotated axes are almost identical and describe the same fast-slow continuum.

a

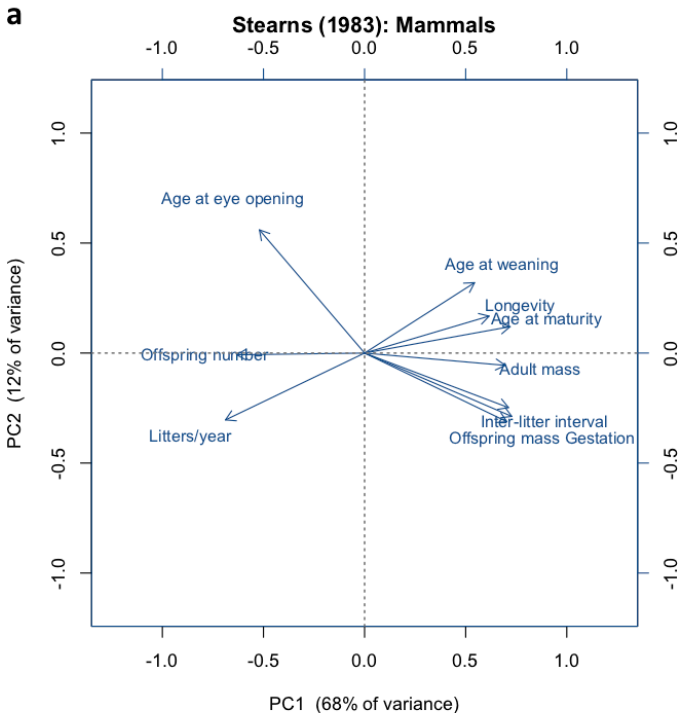

C

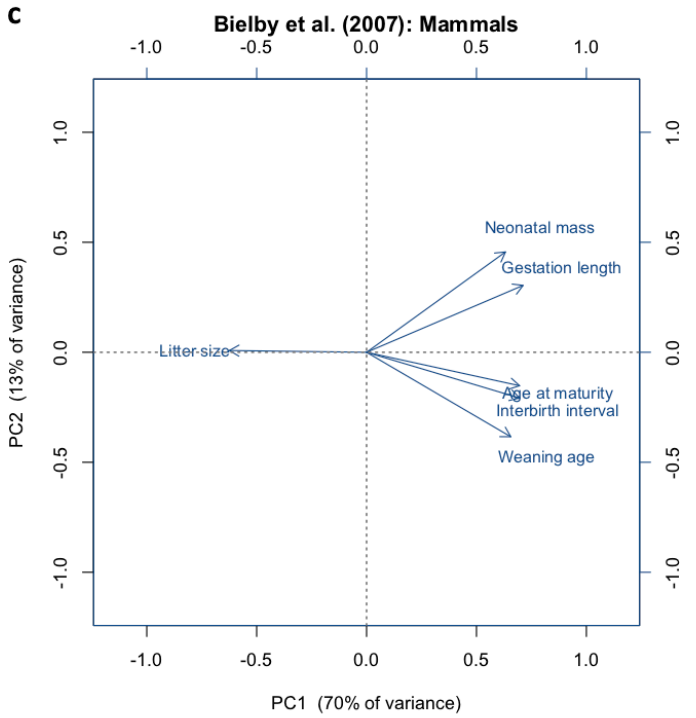

b

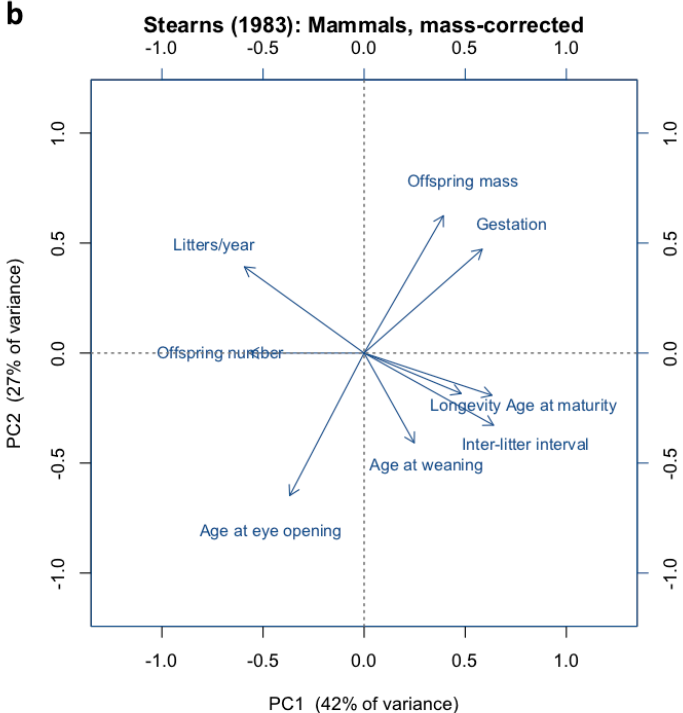

d

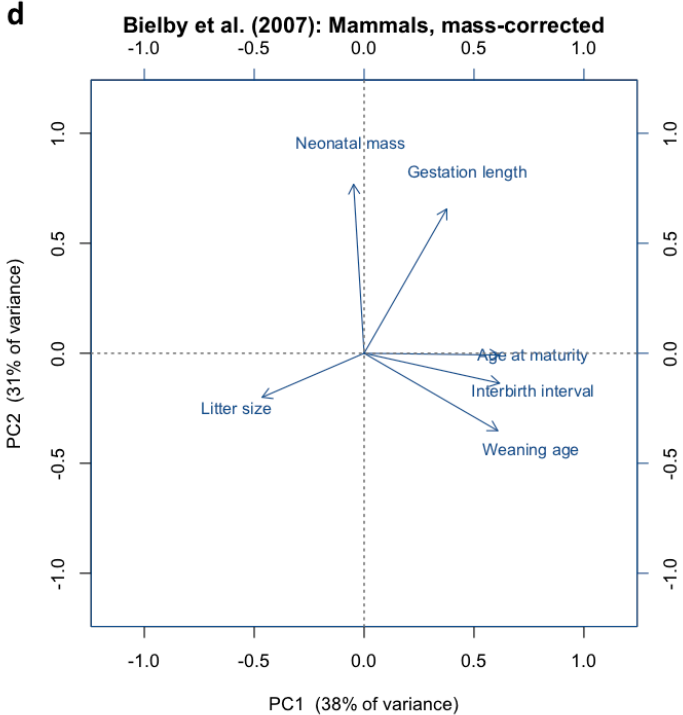

Figure S1.2. Unrotated loading plots based on Stearns (1983) and Bielby et al. (2007; the data were reanalyzed in Del Giudice, 2014). Note that the sign of PC2 loadings in panel (a) is reversed with respect to the other panels. 
a

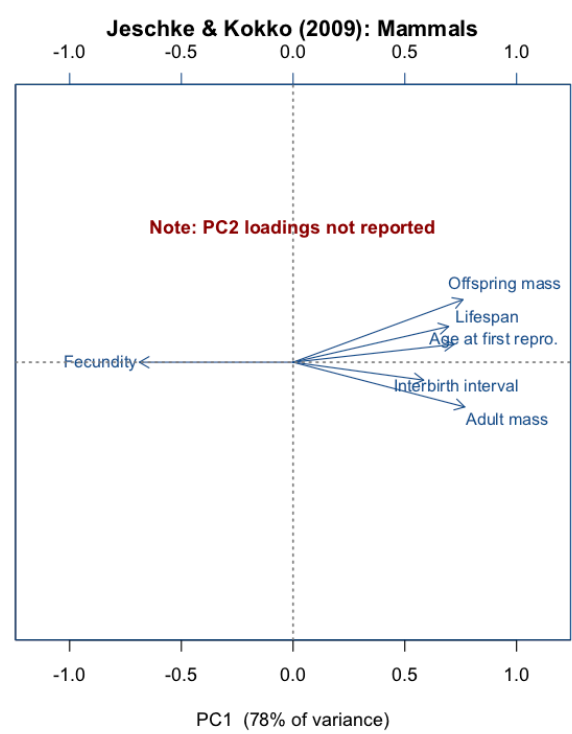

c

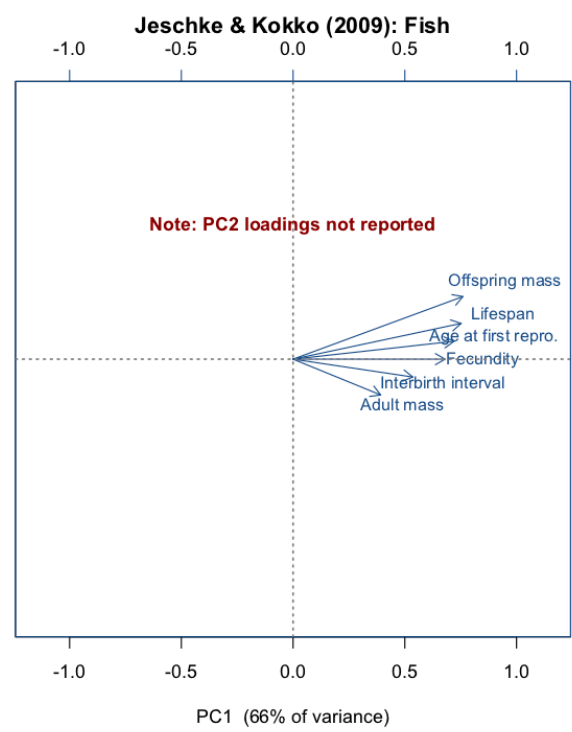

e

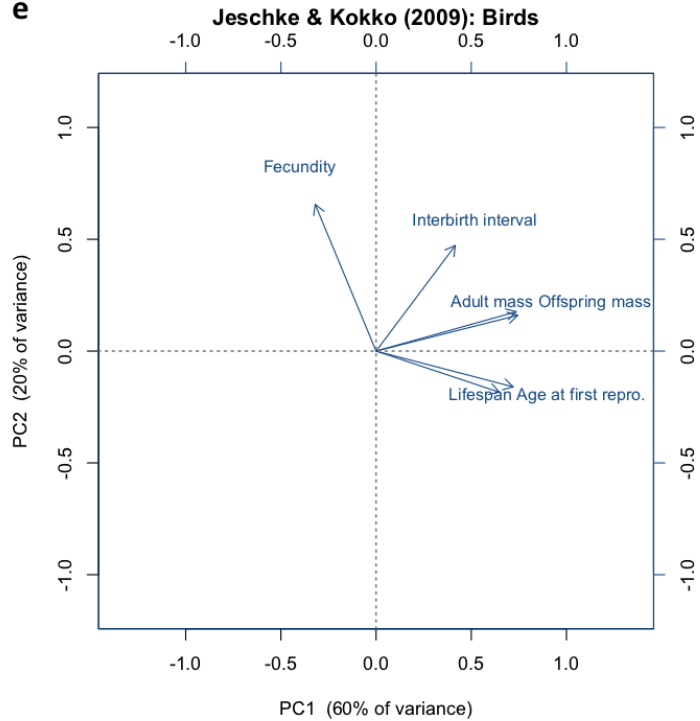

b Jeschke \& Kokko (2009): Mammals, mass-corrected

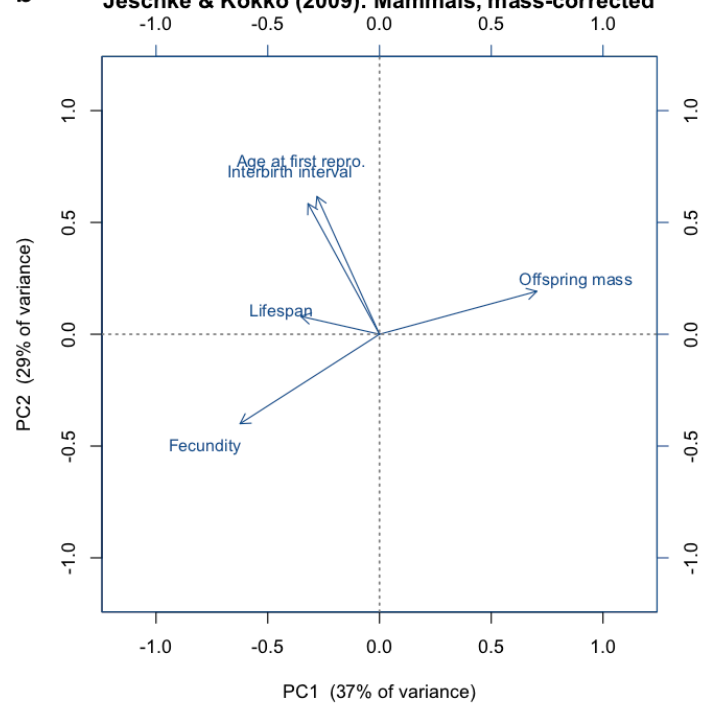

d Jeschke \& Kokko (2009): Fish, mass-corrected

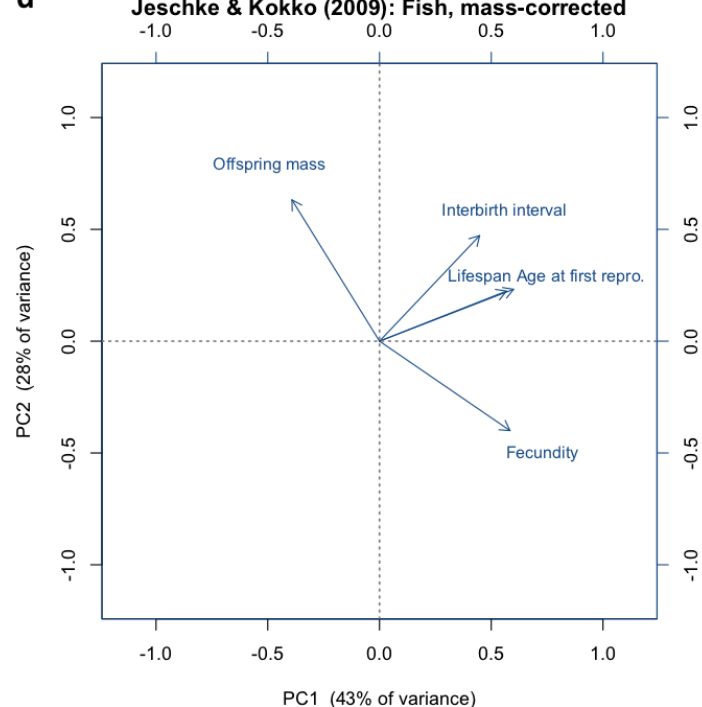

f Jeschke \& Kokko (2009): Birds, mass-corrected

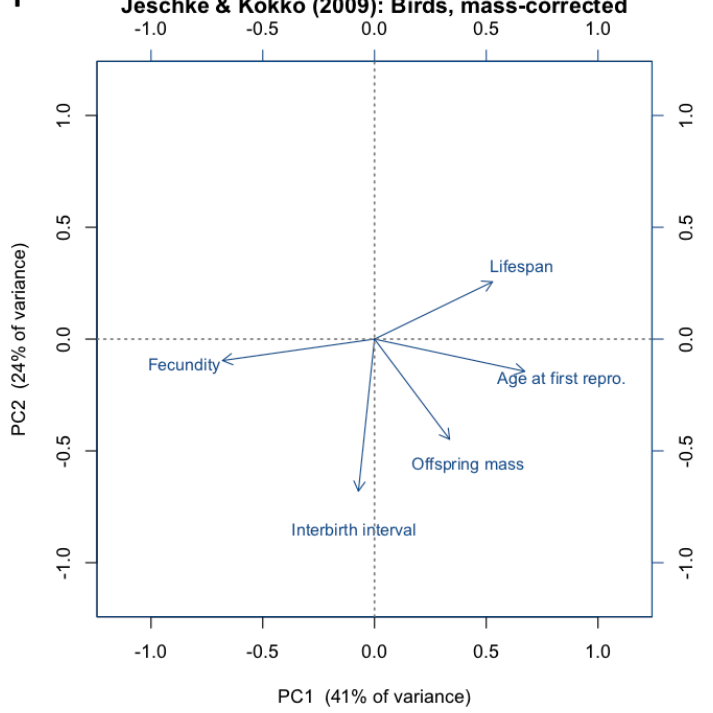

Figure S1.3. Unrotated loading plots based on Jeschke \& Kokko (2009). 

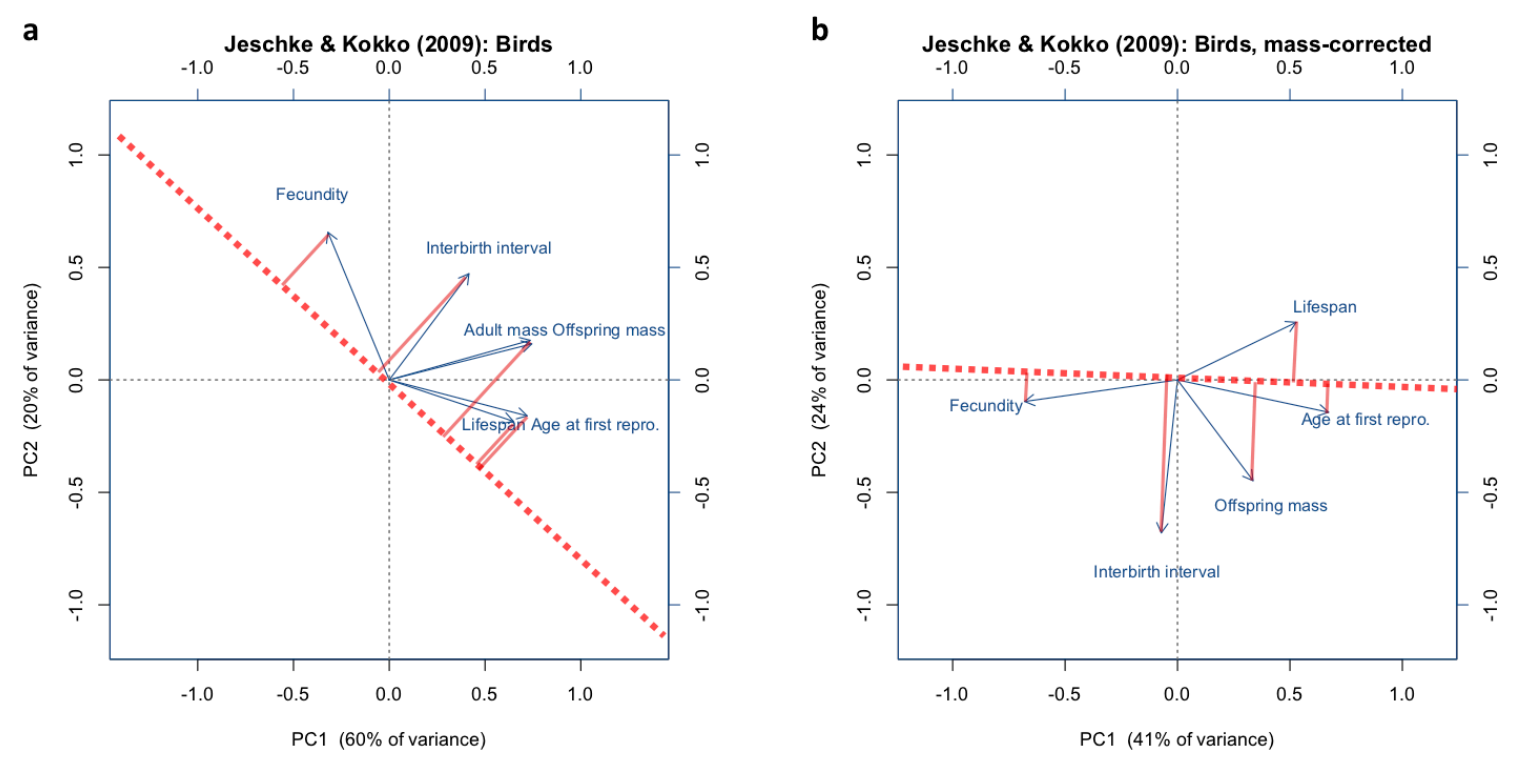

Figure S1.4. Unrotated loading plots of bird life histories based on Jeschke \& Kokko (2009), with rotated axes superimposed in red. The new axes are rotated so as to align with fertility (here "fecundity") and age at first reproduction, and describe a fast-slow continuum very similar to the one observed in mammals. While the original solutions in (a) and (b) look superficially different, the rotated axes are almost identical, as can be seen from the loadings of the variables on the new axes (solid red lines).

In sum, researchers interested in life history variation should be careful about using standard rotation methods. These methods are designed to approximate a simple structure, and are unable to recover general axes of variation such as the fast-slow continuum. Standard orthogonal rotations such as varimax are probably best avoided in this context; an alternative approach is to use oblique rotations (e.g., oblimin, geomin, promax) to obtain correlated components, then subject those components to further dimension reduction. For example, when I applied an oblique rotation to the data in Bielby et al. (2007), the correlation between the two rotated components was .65 in the uncorrected solution and .32 in the mass-corrected solution (Del Giudice, 2014). However, the first unrotated component is usually a good description of the fast-slow continuum when the latter accounts for a large proportion of the variance. That said, it is always a good idea to inspect loading plots to detect atypical solutions like the one in Figure $\mathrm{S} 1.3 \mathrm{e}$. This is even more crucial when PC1 and PC2 account for similar proportions of variance, as is generally the case in mass-corrected datasets; the resulting solutions tend to be less stable, and the unrotated components may not align with the meaningful axes of variation in the data.

Since all the possible rotations are mathematically equivalent and the standard "simple structure" is not appropriate in this context, an interesting option is to anchor the solution to a biologically relevant criterion. For example, Oli (2004) showed that the ratio of fertility rate to age at first reproduction $(\mathrm{F} / \alpha)$ is a meaningful summary of a species' position on the fast-slow continuum in mammals (most likely, the same applies to birds; see Jeschke \& Koko, 2009; Section 2.1). This suggests that, in ambiguous situations like the one in Figure S1.4, researchers could rotate the first component so that it aligns with age at first reproduction and fertility rate, or another theoretically justified set of variables (for example by maximizing the sum of the squared loadings of those variables). If the anchoring variables are chosen sensibly, the rotated solution has a good chance of being more interpretable and biologically meaningful than the unrotated one. 

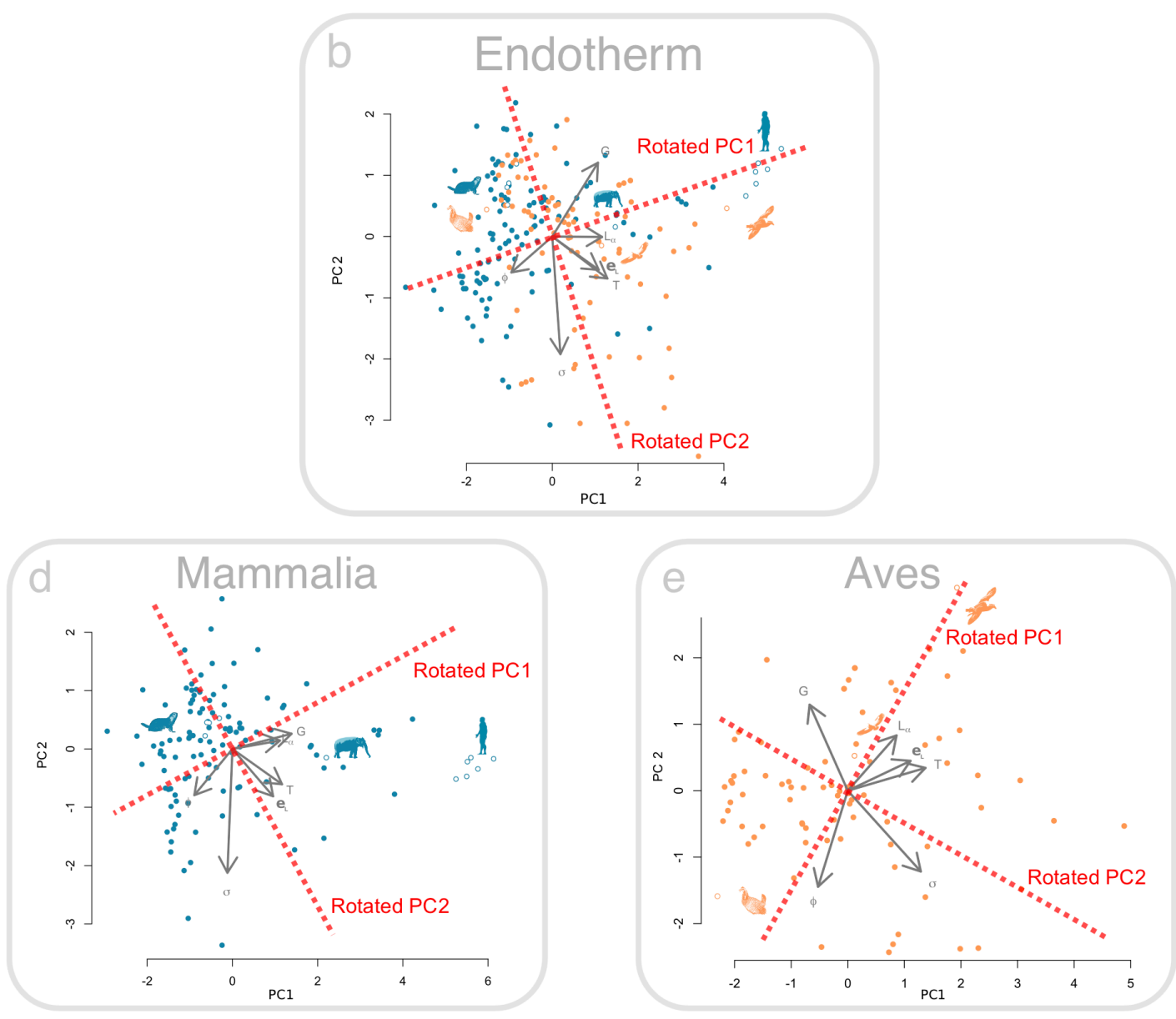

$\begin{array}{ll}\mathbf{e}_{\mathrm{L}} \text { Mean life expectancy post maturity } & \sigma \text { Distribution of mortality risk } \\ \mathrm{L}_{\alpha} \text { Age at first sexual reproduction } & \mathrm{G} \text { Spread of reproduction } \\ \Phi \text { Mean sexual reproductive rate } & \mathrm{T} \text { Generation time }\end{array}$

Figure S1.5. PCA plots from Supplementary Figure 2 in Healy et al. (2019; mass- and phylogenycorrected), with rotated axes superimposed in red. The components have been rotated so that PC1 aligns with age at first reproduction and fertility rate (see Oli, 2004). Adapted with permission from Healy et al. (2019).

To further illustrate this approach, Figure S1.5 shows PCA plots from the recent largescale study by Healy et al. (2019). Consistent with the classic fast-slow continuum, the first unrotated component (PC1) summarizes variation in age at first reproduction, life expectancy, and generation time across a wide range of species. However, reproductive rate shows the expected negative loading on PC1 only in endotherms and mammals, but not in birds (and ectotherms; not shown here). This and similar findings led the authors to conclude that the fastslow continuum is only partially supported across taxonomic groups. An alternative possibility is that PCA failed to precisely identify the biologically meaningful axes of variation in these datasets, perhaps owing to the particular mix of species and/or variables included in the analyses. Note that the data were corrected for body mass and phylogeny; in birds, the resulting PC1 and PC2 account for similar proportions of variance ( $46 \%$ and $24 \%$, respectively). Rotating the axes so that $\mathrm{PC} 1$ aligns with fertility rate and age at first reproduction (e.g., the superimposed red axes in Figure S1.5) yields a more interpretable solution. The rotated PC1 describes a classic fast-slow 
continuum in endotherms, birds, and (somewhat less clearly) mammals. Taken together, the new solutions show that the apparent discrepancy between fast-slow continua in endotherms and birds reflects an artifact of rotation, not a true taxonomic difference.

\section{References}

Abdi, H. (2007). RV coefficient and congruence coefficient. In N. Salkind (Ed.), Encyclopedia of measurement and statistics (pp. 849-853). Thousand Oaks, CA: Sage.

Bielby, J., Mace, G. M., Bininda-Emonds, O. R., Cardillo, M., Gittleman, J. L., Jones, K. E., ... \& Purvis, A. (2007). The fast-slow continuum in mammalian life history: An empirical reevaluation. The American Naturalist, 169, 748-757.

Browne, M. W. (2001). An overview of analytic rotation in exploratory factor analysis. Multivariate Behavioral Research, 36, 111-150

Darton, R. A. (1980). Rotation in factor analysis. The Statistician, 29, 167-194.

Del Giudice, M. (2014). A tower unto Heaven: Toward an expanded framework for psychopathology. Psychological Inquiry, 25, 394-413.

Healy, K., Ezard, T. H., Jones, O. R., Salguero-Gómez, R., \& Buckley, Y. M. (2019). Animal life history is shaped by the pace of life and the distribution of age-specific mortality and reproduction. Nature Ecology \& Evolution, 3, 1217-1224.

Jeschke, J. M., \& Kokko, H. (2009). The roles of body size and phylogeny in fast and slow life histories. Evolutionary Ecology, 23, 867-878.

Lykken, D. T. (1971). Multiple factor analysis and personality research. Journal of Experimental Research in Personality, 5, 161-170.

Salguero-Gómez, R. (2017). Applications of the fast-slow continuum and reproductive strategy framework of plant life histories. New Phytologist, 213, 1618-1624.

Sass, D. A., \& Schmitt, T. A. (2010). A comparative investigation of rotation criteria within exploratory factor analysis. Multivariate Behavioral Research, 45, 73-103.

Stearns, S. C. (1983). The influence of size and phylogeny on patterns of covariation among lifehistory traits in the mammals. Oikos, 41, 173-187. 


\section{S2. Balancing selection, temporal fluctuations, and the genetics of individual differences}

The assumption that individual differences in life history-related traits can be maintained by balancing selection has been criticized because of its apparent inconsistency with the genomic data. Specifically, critics argue that balancing selection should lead to an overwhelming contribution from alleles of high frequency (Verweij et al., 2012; Zietsch \& Sidari, 2020) and/or large phenotypic effect (Penke et al., 2007). In contrast, the bulk of variation in polygenic traits is due to common variants of small effect and rare mutations/structural variants. More generally, genomic studies have found relatively few loci matching the expected signatures of balancing selection (but see Bitarello et al., 2018); thus, many authors regard balancing selection as marginal or limited to special gene classes (e.g., immune-related genes; Sella \& Barton, 2019).

There are several problems with this view (see also Penke \& Jokela, 2016). Statistical tests of balancing selection have important limitations, including low detection power and high error rates; more importantly, they can only detect long-term instances of balancing selection that may be the exception rather than the rule (Fijarczyk \& Babik, 2015). The "signatures" of balancing selection in the genome emerge on different time scales, and only if the same alleles are maintained at equilibrium for many generations. In humans, an excess of high-frequency variants is only expected to become apparent after hundreds of thousands of years of consistent selection on the same alleles (that is, a number of generations of about 0.4 times the effective population size; see Fijarczyk \& Babik, 2015). In view of the rapid evolution and striking ecological expansion of humans over the past tens of thousands of years, this kind of scenario seems unlikely. The process is even slower — and leaves even weaker traces - when balancing selection involves antagonistic effects, a plausible scenario for life history trade-offs (Connallon \& Clark, 2013; Fijarczyk \& Babik, 2015). When traits are highly polygenic and dominated by small effects, adaptation typically takes place via "soft" or incomplete sweeps, and subtle changes in allele frequency across multiple genes (polygenic adaptation; see Hermisson \& Pennings, 2005, 2017; Messer et al., 2016; Pritchard et al., 2010). Under these conditions, balancing selection should often proceed via transient episodes on the background of a shifting genetic architecture, with few instances of the long-term equilibria envisioned by classic models. The signatures of this kind of process are exceedingly hard to differentiate from those of recent directional selection or even neutrality, which probably explains the dearth of genomic findings (Fijarczyk \& Babik, 2015; Vitti et al., 2013; Yeaman, 2015).

Similarly, the expectation that alleles maintained by balancing selection should have large phenotypic effects (e.g., Penke et al., 2007) depends on the unrealistic modeling assumption that a single gene can take full control of expression level of a trait (e.g., Bürger, 2002; Kopp \& Hermisson, 2006). Of course, such a trait would be extremely fragile against mutations and other disturbances, and it is highly unlikely that most behavioral and physiological mechanisms - with their multiple layers of redundancy, extensive feedback regulation, and modular organization-would ever evolve in this way (see Del Giudice, 2012).

Historically, the skepticism about balancing selection has been even stronger for hypotheses that involve fluctuations over time (see Messer et al., 2016). Early models of variable selection indicated that temporal fluctuations are generally unable to maintain genetic variation in a population, in contrast with spatial variation (e.g., Frank \& Slatkin, 1990; Hedrick et al., 1976; Hedrick, 1986). However, this initial finding has been overturned by a new generation of models. If traits are highly polygenic and subject to recurrent mutations, temporal fluctuations 
can be effective at maintaining variation (Bürger \& Gimelfarb, 2002). Even more importantly, the early models assumed discrete and nonoverlapping generations; as a result, the entire population is exposed to negative selection at the same time and genetic variation gets rapidly depleted. But in species with overlapping generations (including humans) and/or maternal effects that buffer juveniles from temporary negative selection, polymorphisms can be easily maintained as the environment fluctuates ("storage effects;" Bertram \& Masel, 2019; Ellner \& Hairston, 1994; Ellner, 1996; Ellner \& Sasaki, 1996; Hedrick, 1995; Yamamichi \& Hoso, 2016). Dominance reversal is another potential mechanism that increases the effectiveness of fluctuating selection, and may plausibly occur in the context of life history trade-offs (Bertram \& Masel, 2019; see Connallon \& Chenoweth, 2019). The distribution of environmental states over time is also important: for example, some models suggest that fluctuations maintain variation at a larger number of loci if they follow a heavy-tailed distribution (Ellner \& Sasaki, 1996). Note that these facilitating factors have been identified in theoretical models, but there is still no empirical evidence regarding their role (or lack thereof) in our species.

In sum, the genetic architecture of life history-related traits in humans and other animals is compatible with a mixture of mutation-selection balance and balancing selection (e.g., Charlesworth, 2015). Contrary to widespread assumptions, balancing selection can be sustained by temporal variation, and in realistic conditions does not necessarily result in a large contribution of intermediate-frequency alleles (whether of small or large phenotypic effect). Some recent studies have found cues of long-term balancing selection across the human genome (e.g., Bitarello et al., 2018); but there are reasons to believe that many if not most instances of balancing selection have left subtle, ambiguous traces that cannot be reliably detected using current genomic tools.

\section{References}

Bertram, J., \& Masel, J. (2019). Different mechanisms drive the maintenance of polymorphism at loci subject to strong versus weak fluctuating selection. Evolution, 73, 883-896.

Bitarello, B. D., de Filippo, C., Teixeira, J. C., Schmidt, J. M., Kleinert, P., Meyer, D., \& Andrés, A. M. (2018). Signatures of long-term balancing selection in human genomes. Genome Biology and Evolution, 10, 939-955.

Bürger, R. (2002). Additive genetic variation under intraspecific competition and stabilizing selection. Journal of Theoretical Population Biology, 61, 197-213.

Bürger, R., \& Gimelfarb, A. (2002). Fluctuating environments and the role of mutation in maintaining quantitative genetic variation. Genetics Research, 80, 31-46.

Charlesworth, B. (2015). Causes of natural variation in fitness: Evidence from studies of Drosophila populations. Proceedings of the National Academy of Sciences USA, 112, 1662-1669.

Connallon, T., \& Chenoweth, S. F. (2019). Dominance reversals and the maintenance of genetic variation for fitness. PLoS Biology, 17, e3000118.

Connallon, T., \& Clark, A. G. (2013). Antagonistic versus nonantagonistic models of balancing selection: Characterizing the relative timescales and hitchhiking effects of partial selective sweeps. Evolution, 67, 908-917.

Del Giudice, M. (2012). Sex ratio dynamics and fluctuating selection on personality. Journal of Theoretical Biology, 297, 48-60.

Ellner, S. (1996). Environmental fluctuations and the maintenance of genetic diversity in age or stage-structured populations. Bulletin of Mathematical Biology, 58, 103-127. 
Ellner, S., \& Hairston, N. G., Jr., (1994). Role of overlapping generations in maintaining genetic variation in a fluctuating environment. American Naturalist, 143, 403-417.

Ellner, S., \& Sasaki, A. (1996). Patterns of genetic polymorphism maintained by fluctuating selection with overlapping generations. Theoretical Population Biology, 50, 31-65.

Fijarczyk, A., \& Babik, W. (2015). Detecting balancing selection in genomes: Limits and prospects. Molecular Ecology, 24, 3529-3545.

Frank, S. A., \& Slatkin, M. (1990). Evolution in a variable environment. The American Naturalist, 136, 244-260.

Hedrick, P. W. (1986). Genetic polymorphism in heterogeneous environments: A decade later. Annual Review of Ecology and Systematics, 17, 535-566.

Hedrick, P. W. (1995). Genetic polymorphism in a temporally varying environment: Effects of delayed germination or diapause. Heredity, 75, 164-170.

Hedrick, P. W., Ginevan, M. E., \& Ewing, E. P. (1976). Genetic polymorphism in heterogeneous environments. Annual Review of Ecology and Systematics, 7, 1-33.

Hermisson, J., \& Pennings, P. S. (2005). Soft sweeps: Molecular population genetics of adaptation from standing genetic variation. Genetics, 169, 2335-2352.

Hermisson, J., \& Pennings, P. S. (2017). Soft sweeps and beyond: Understanding the patterns and probabilities of selection footprints under rapid adaptation. Methods in Ecology and Evolution, 8, 700-716.

Kopp, M., \& Hermisson, J. (2006). Evolution of genetic architecture under frequency-dependent disruptive selection. Evolution, 60, 1537-1550.

Messer, P. W., Ellner, S. P., \& Hairston Jr, N. G. (2016). Can population genetics adapt to rapid evolution? Trends in Genetics, 32, 408-418.

Penke, L., Denissen, J. J., \& Miller, G. F. (2007). The evolutionary genetics of personality. European Journal of Personality, 21, 549-587.

Penke, L., \& Jokela, M. (2016). The evolutionary genetics of personality revisited. Current Opinion in Psychology, 7, 104-109.

Pritchard, J. K., Pickrell, J. K., \& Coop, G. (2010). The genetics of human adaptation: Hard sweeps, soft sweeps, and polygenic adaptation. Current Biology, 20, R208-R215.

Sella, G., \& Barton, N. H. (2019). Thinking about the evolution of complex traits in the era of genome-wide association studies. Annual Review of Genomics and Human Genetics, doi: 10.1146/annurev-genom-083115-022316

Verweij, K. J., Yang, J., Lahti, J., Veijola, J., Hintsanen, M., Pulkki-Råback, L., ... \& Taanila, A. (2012). Maintenance of genetic variation in human personality: Testing evolutionary models by estimating heritability due to common causal variants and investigating the effect of distant inbreeding. Evolution, 66, 3238-3251.

Vitti, J. J., Grossman, S. R., \& Sabeti, P. C. (2013). Detecting natural selection in genomic data. Annual Review of Genetics, 47, 97-120.

Yamamichi, M., \& Hoso, M. (2017). Roles of maternal effects in maintaining genetic variation: Maternal storage effect. Evolution, 71, 449-457.

Yeaman, S. (2015). Local adaptation by alleles of small effect. The American Naturalist, 186, S74-S89.

Zietsch, B. P., \& Sidari, M. J. (2019). A critique of life history approaches to human trait covariation. Evolution and Human Behavior, doi: 10.1016/j.evolhumbehav.2019.05.007 


\section{S3. Trade-offs and life history-related traits in humans}

\section{Maturation timing and the current-future reproduction trade-off}

The human life history literature has devoted a great deal of attention to the timing of sexual maturation (e.g., Belsky et al., 1991; Belsky, 2012; James \& Ellis, 2013). Maturation timing is theoretically important as a mediator of trade-off between somatic versus reproductive effort and current versus future reproduction; and there are genetic as well as phenotypic correlations between puberty, age of sexual debut, age of first birth, and (female) fertility (e.g., Barban et al., 2016; Briley et al., 2017; Dunbar et al., 2008; Ibitoye et al., 2017; Lawn et al., 2019; Tropf et al., 2015; Udry \& Cliquet, 1982). However, the net effect of puberty timing on fertility is limited, and the link between maturation and the start of reproduction can be weakened by various social factors (e.g., marriage practices; Borgerhoff Mulder, 1989; Udry \& Cliquet, 1982). Maturation is also strongly affected by nutrition, with the result that allocations to current versus future reproduction are confounded with adaptive responses to improved physical condition and energy availability (e.g., Kyweluk et al., 2018; Stearns \& Rodrigues, 2020). Even if maturation timing is not the main mediator of reproductive allocations in humans, it can play other roles in a broader life history perspective; for example, a slow and protracted developmental schedule can favor the acquisition of knowledge and skills - forms of "embodied capital" that pay off later in life (Berger-Tal et al., 2014; Eliassen et al., 2007; Kaplan et al., 2000).

More generally, our species has a long reproductive window, considerable flexibility in the timing of reproduction, and an extended period of care and provision for dependent offspring. These factors converge in reducing the relative importance of the current-future reproduction trade-off - particularly regarding the timing of sexual maturation — and increasing that of other trade-offs involving mating, parenting, and offspring quality (see below). This applies particularly to men, who are not limited by the physiological constraints of pregnancy, lactation, and menopause, and whose reproductive success depends more critically on the age of their partners than on their own (Borgerhoff Mulder \& Ross, 2019; Ponzi et al., 2020). Especially in growing populations, an earlier start of reproduction can boost a woman's fitness (Jones \& Bird, 2014), and may not entail sacrificing the quality of parental investment if she can count on a committed partner and/or support from kin. In total, the links between early maturation and other traits related to "fast" strategies should be stronger in females than in males; but even in females, correlations are likely to be small, owing to the partially stochastic nature of maturational events, the confounding effects of condition and nutrition, and the flexibility of human reproduction.

\section{The centrality of the mating-parenting trade-off}

The remarkable intensity of parental investment in our species suggests that qualityquantity trade-offs should play a major role in human life history strategies. The evidence, however, is contradictory and hard to interpret (Lawson \& Borgerhoff Mulder, 2016). There are several reasons for this. First, most studies have used child survival as the metric of quality (see Lawson \& Borgerhoff Mulder, 2016), but humans can contribute to the eventual reproductive success of their offspring by other means-for example by transmitting material resources, knowledge, status, and social networks (Jones, 2015). Second, and regardless of the definition of quality, parents with better health and more resources can typically afford to have more children; at least in traditional societies, this could easily mask the existence of a trade-off (Lawson \& 
Borgerhoff Mulder, 2016). Third, when population size is not stable (e.g., in a growing population), fitness does not just depend on the number of children but also on the timing of childbearing; when timing is accounted for, trade-offs involving fertility become more apparent (Jones \& Bird, 2014). Finally, children are not just a cost on parents, but can actively contribute to childcare and other subsistence activities, thus lessening the strength of the trade-off (Lawson \& Borgerhoff Mulder, 2016).

In our species, the quality-quantity trade-off overlaps to a large extent with that between mating and parenting effort, which in turn is intensified by the long duration of juvenile dependency (Borgerhoff Mulder \& Ross, 2019; Lawson \& Borgerhoff Mulder, 2016; Winking \& Koster, 2015). Stereotypes of mating- versus parenting-oriented men are salient and easily recognized across cultures (Kruger et al., 2003, 2015). I have argued that the mating-parenting trade-off occupies a central place in the structure of the human fast-slow continuum - especially in men, who are less constrained by the timing of reproduction, and can potentially sire many offspring with little or no parental investment (see Copping \& Richardson, 2020; Del Giudice, 2018). As noted above, even differences in maturation timing may partly reflect allocations to embodied capital in view of later competition for status and mating.

\section{Multiple pathways to mating and parenting}

In most animals, the main currencies of parental effort are energy/nutrient transfer and protection from danger. As noted earlier, humans can transfer multiple kinds of resources to their offspring; particularly since the invention of agriculture, people can enhance the fitness of their children - and often their grandchildren - by endowing them with land, cattle, money, and other forms of wealth (Borgerhoff Mulder et al., 2019; Jones, 2015). This opens up an alternative pathway to parental effort: especially for men, investment in parenting may involve the accumulation and transmission of wealth in addition to (or in place of) direct caregiving and provisioning.

Another important innovation in our species is the evolution of dual status hierarchies based on dominance and prestige (Henrich \& Gil-White, 2001). The relevant point is that status and influence can be gained not only with the threat of physical force, but also with the possession of valued skills and knowledge. Again, this multiplies the pathways to mating success - particularly for men, who benefit more consistently from status and wealth (Nettle \& Pollet, 2008; Pérusse, 1993; Stulp et al., 2016; von Rueden \& Jaeggi, 2016; Winegard et al., 2018). Alternatively (or concurrently), the material benefits of high status can be transferred to one's family and offspring and thus channeled into indirect parenting effort.

A final aspect to consider is the nature of human courtship and mate choice. In our species, mate choice is not unidirectional but has a marked reciprocal component (StewartWilliams \& Thomas, 2013). Both sexes court potential partners by displaying desirable qualities, including intelligence and creativity (e.g., Conroy-Beam et al., 2019; Gabora \& Kaufman, 2010; Winegard et al., 2018). At the same time, it is important to keep in mind that, in most societies, mates are not chosen freely but under various degrees of parental influence or outright control (Apostolou, 2010, 2017). Success in courtship can be achieved by appealing not just to the preferences of the partners themselves, but also to those of their parents - who are likely to place more value on traits that advertise cooperation and reliable parenting (see Apostolou, 2017; Buunk et al., 2008). 
In sum, the mating-parenting tradeoff in humans can take a variety of forms. Allocations to mating and parenting can be realized through multiple pathways, which in turn should reward different combinations of cognitive and behavioral traits. For this reason, the fast-slow continuum is unlikely to be associated with a unitary set of traits; instead, fast and slow strategies may comprise a range of variants of "profiles," with similar implications for basic trade-offs but distinct psychological mediators (Del Giudice, 2018; Figure S3.1).

\section{Mapping human life history-related traits}

In order to use the fast-slow continuum as a heuristic for individual differences, it is useful to distinguish between basic life history traits and the broader suite of behavioral, physiological, and morphological phenotypes that contribute to determining them (Section 3.2). I suggested the label "life history-related" for traits that (a) are intra-individually stable enough to be treated as individual differences variables; (b) covary with basic life history traits and/or other outcomes of life history allocations; and (c) plausibly contribute to mediating those allocations, or function as proxies of traits that do. Validating putative life history-related traits with survival and reproduction data is fraught with difficulties, especially in post-demographic transition societies with easy access to contraception, abortion, and modern medicine. For example, as contraception breaks the link between mating and reproduction (e.g., Pérusse, 1993), people with a strong desire for sexual variety may postpone or forgo reproduction, increasing the relative fertility of those with traits that promote long-term commitment and a desire for children (e.g., Woodley of Menie et al., 2017). But even in traditional societies, fitness is not just determined by the number of children or the number of mates: the timing of reproduction can be critical when populations are growing or shrinking, and the quality of mates can be just as important as their number (see Borgerhoff Mulder \& Ross, 2019; Jones \& Bird, 2014). An underappreciated challenge of doing research on college students is that few of them have children: some psychological aspects of the mating-parenting trade-off may not be fully expressed until people become parents and experience the constraints imposed by childbearing and/or caregiving, on top of the physiological changes associated with parenthood. Studies based on college students are likely to underestimate the strength of the trade-off (see e.g., Kruger, 2017). The validation of life history-related traits is a truly intricate task, and the existing data are still tentative in many ways.

In some research contexts, one may want to select a subset of traits that can serve as "markers" of fast versus slow strategies. In a recent book on evolutionary psychopathology (Del Giudice, 2018), I proposed the following as "core" behavioral markers of life history strategies: conscientiousness and honesty-humility; impulsivity, present vs. future orientation, sensation seeking, and risk-taking; timing of sexual development; restricted vs. unrestricted sociosexuality; long-term mating orientation; stable vs. unstable romantic attachments; exploitative vs. cooperative attitudes; and sensitivity to sexual/moral disgust. For a full exposition see Del Giudice (2018). These traits are meant to be used together as convergent but fallible markers of life history strategy. Empirical correlations involving some of these traits can be moderated by various factors (e.g., attractiveness, nutrition; Copping et al., 2014; James \& Ellis, 2013), and may differ somewhat between alternative profiles.

This list of markers is deliberately selective, since many aspects of personality and individual differences have complex or less than straightforward links with life history tradeoffs. For example, extraversion and openness to experience are not included in the list because their facets show contrasting relations with mating behaviors and other life history-related traits (e.g., Holtzman \& Strube, 2013; Manson, 2017). The general factor of personality (GFP) is also 
excluded, partly because of its low resolution and partly because it is strongly contaminated by evaluative biases (see Davies et al., 2015; Dunkel et al., 2016). Agreeableness is generally associated with "slow" traits and outcomes, but the model I propose includes a slow life history profile with moderate/low agreeableness. While the list includes conscientiousness, the role of this broad personality trait should be reconsidered and defined more precisely. Measures of conscientiousness often yield contradictory or paradoxical findings, because they include elements of performance (e.g., succeeding in being orderly and punctual) that are subject to evaluative distortions (e.g., perfectionism) and may not track motivation very closely (e.g., Mike et al., 2018; Mõttus et al., 2018).

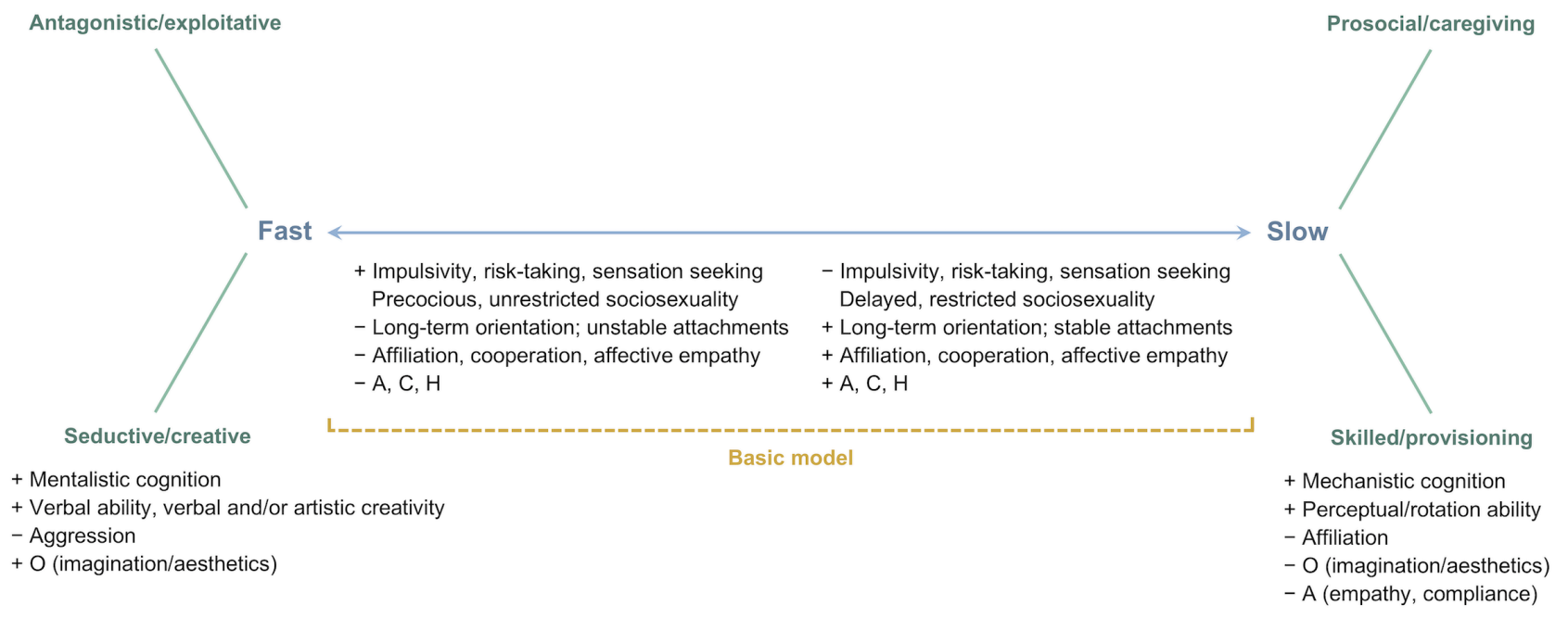

Extended model

Figure S3.1. An extended model of life history-related traits in humans (adapted with permission from Del Giudice, 2018). The basic model only distinguishes between fast and slow strategies; the extended model postulates the existence of alternative profiles defined by specific clusters of behavioral and cognitive traits. $\mathrm{A}=$ agreeableness; $\mathrm{C}=$ conscientiousness; $\mathrm{H}=$ honesty-humility; $\mathrm{O}=$ openness to experience.

By way of illustration, Figure S3.1 shows my recent proposal for an extended model of life history-related traits in humans, based on the idea of multiple profiles. In addition to the clusters of traits envisioned by the standard or "basic" model that informs the current literature (here labeled prosocial/caregiving and antagonistic/exploitative), I described two additional profiles: a creative/seductive profile associated with narcissistic and psychotic-like traits, and a male-biased skilled/provisioning profile associated with autistic-like traits (for details see Del Giudice, 2018). If the model is broadly correct, the profiles it describes cannot be identified by standard factor-analytic methods. Popular "life history" questionnaires such as the Mini-K (Figueredo et al., 2006) mostly reflect a combination of agreeableness, conscientiousness, extraversion, and - to a smaller extent-neuroticism (Manson, 2017; Olderbak et al., 2014), and correlate weakly with indicators of mating effort (Copping et al., 2017). According to the model in Figure S3.1, these scales map on the fast-slow continuum in a partial and imperfect fashion; for example, one would expect them to misclassify people who match the skilled/provisioning profile as "fast" strategists because of their comparatively low agreeableness and sociability (see Del Giudice, 2018). 


\section{References}

Apostolou, M. (2010). Sexual selection under parental choice in agropastoral societies. Evolution and Human Behavior, 31, 39-47.

Apostolou, M. (2017). Sexual selection in Homo sapiens: Parental control over mating and the opportunity cost of free mate choice. Springer.

Barban, N., Jansen, R., De Vlaming, R., Vaez, A., Mandemakers, J. J., Tropf, F. C., ... \& Tragante, V. (2016). Genome-wide analysis identifies 12 loci influencing human reproductive behavior. Nature Genetics, 48, 1462-1472.

Belsky, J., Steinberg, L., \& Draper, P. (1991). Childhood experience, interpersonal development, and reproductive strategy: An evolutionary theory of socialization. Child Development, 62, 647-670.

Belsky, J. (2012). The development of human reproductive strategies: Progress and prospects. Current Directions in Psychological Science, 21, 310-316.

Berger-Tal, O., Nathan, J., Meron, E., \& Saltz, D. (2014). The exploration-exploitation dilemma: A multidisciplinary framework. PLoS ONE, 9, e95693.

Borgerhoff Mulder, M. (1989). Menarche, menopause and reproduction in the Kipsigis of Kenya. Journal of Biosocial Science, 21, 179-192.

Borgerhoff Mulder, M., \& Ross, C. T. (2019). Unpacking mating success and testing Bateman's principles in a human population. Proceedings of the Royal Society of London B, 286, 20191516.

Borgerhoff Mulder, M., Towner, M. C., Baldini, R., Beheim, B. A., Bowles, S., Colleran, H., ... \& Scelza, B. A. (2019). Differences between sons and daughters in the intergenerational transmission of wealth. Philosophical Transactions of the Royal Society of London B, 374, 20180076.

Buunk, A. P., Park, J. H., \& Dubbs, S. L. (2008). Parent-offspring conflict in mate preferences. Review of General Psychology, 12, 47-62.

Conroy-Beam, D., Roney, J. R., Lukaszewski, A. W., Buss, D. M., Asao, K., Sorokowska, A., ... \& Alm, C. (2019). Assortative mating and the evolution of desirability covariation. Evolution and Human Behavior, 40, 479-491.

Copping, L. T., Campbell, A., \& Muncer, S. (2014). Psychometrics and life history strategy: The structure and validity of the high K strategy scale. Evolutionary Psychology, 12, 200222.

Copping, L. T., Campbell, A., Muncer, S., \& Richardson, G. B. (2017). The psychometric evaluation of human life histories: A reply to Figueredo, Cabeza de Baca, Black, Garcia, Fernandes, Wolf, and Woodley (2015). Evolutionary Psychology, 1474704916663727.

Copping, L. T., \& Richardson, G. B. (2020). Studying Sex Differences in Psychosocial Life History Indicators. Evolutionary Psychological Science, 6, 47-59.

Davies, S. E., Connelly, B. S., Ones, D. S., \& Birkland, A. S. (2015). The General Factor of Personality: The "Big One," a self-evaluative trait, or a methodological gnat that won't go away? Personality and Individual Differences, 81, 13-22.

Del Giudice, M. (2018). Evolutionary psychopathology: A unified approach. New York: Oxford University Press.

Dunbar, J., Sheeder, J., Lezotte, D., Dabelea, D., \& Stevens-Simon, C. (2008). Age at menarche and first pregnancy among psychosocially at-risk adolescents. American Journal of Public Health, 98, 1822-1824. 
Dunkel, C. S., van der Linden, D., Brown, N. A., \& Mathes, E. W. (2016). Self-report based general factor of personality as socially-desirable responding, positive self-evaluation, and social-effectiveness. Personality and Individual Differences, 92, 143-147.

Eliassen, S., Jørgensen, C., Mangel, M., \& Giske, J. (2007). Exploration or exploitation: Life expectancy changes the value of learning in foraging strategies. Oikos, 116, 513-523.

Figueredo, A. J., Vásquez, G., Brumbach, B. H., Schneider, S. M. R., Sefcek, J. A., Tal, I. R., et al. (2006). Consilience and Life History Theory: From genes to brain to reproductive strategy. Developmental Review, 26, 243-275.

Gabora, L., \& Kaufman, S. B. (2010). Evolutionary approaches to creativity. In J.C. Kaufman \& R. J. Sternberg (Eds.), The Cambridge handbook of creativity (pp. 279-300). New York: Cambridge University Press.

Henrich, J., \& Gil-White, F. J. (2001). The evolution of prestige: Freely conferred deference as a mechanism for enhancing the benefits of cultural transmission. Evolution and Human Behavior, 22, 165-196.

Holtzman, N. S., \& Strube, M. J. (2013). Above and beyond short-term mating, long-term mating is uniquely tied to human personality. Evolutionary Psychology, 11, 147470491301100514.

Ibitoye, M., Choi, C., Tai, H., Lee, G., \& Sommer, M. (2017). Early menarche: A systematic review of its effect on sexual and reproductive health in low-and middle-income countries. PLoS ONE, 12(, e0178884.

James, J., \& Ellis, B. J. (2013). The development of human reproductive strategies: Toward an integration of life history and sexual selection models. In J. A. Simpson \& L. Campbell (Eds.), The Oxford handbook of close relationships (pp. 771-794). New York: Oxford University Press.

Jones, J. H. (2015). Resource transfers and life history evolution. Annual Review of Anthropology, 44, 513-531.

Jones, J. H., \& Bird, R. B. (2014). The marginal valuation of fertility. Evolution and Human Behavior, 35, 65-71.

Kaplan, H. S., Hill, K., Lancaster, J. B., \& Hurtado, A. M. (2000). A theory of human life history evolution: Diet, intelligence, and longevity. Evolutionary Anthropology, 9, 156-185.

Kruger, D. J. (2017). Brief self-report scales assessing life history dimensions of mating and parenting effort. Evolutionary Psychology, 15, 1474704916673840.

Kruger, D. J., Fisher, M. L., De Backer, C., Kardum, I., Tetaz, M., \& Tifferet, S. (2015). Human life history dimensions in reproductive strategies are intuitive across cultures. Human Ethology Bulletin, 30, 109-120.

Kruger, D. J., Fisher, M., \& Jobling, I. (2003). Proper and dark heroes as dads and cads: Alternative mating strategies in British Romantic literature. Human Nature, 14, 305-317.

Kyweluk, M. A., Georgiev, A. V., Borja, J. B., Gettler, L. T., \& Kuzawa, C. W. (2018). Menarcheal timing is accelerated by favorable nutrition but unrelated to developmental cues of mortality or familial instability in Cebu, Philippines. Evolution and Human Behavior, 39, 76-81.

Lawn, R. B., Sallis, H. M., Taylor, A. E., Wootton, R. E., Smith, G. D., Davies, N. M., ... \& Munafo, M. R. (2019). Schizophrenia risk and reproductive success: A Mendelian randomization study. Royal Society Open Science, 6, 181049. 
Lawson, D. W., \& Borgerhoff Mulder, M. (2016). The offspring quantity-quality trade-off and human fertility variation. Philosophical Transactions of the Royal Society of London B, 371, 20150145.

Manson, J. H. (2017). Are extraversion and openness indicators of a slow life history strategy? Evolution and Human Behavior, 38, 552-560.

Mike, A., King, H., Oltmanns, T. F., \& Jackson, J. J. (2018). Obsessive, compulsive, and conscientious? The relationship between OCPD and personality traits. Journal of Personality, 86, 952-972.

Mõttus, R., Allik, J., \& Realo, A. (2010). An attempt to validate national mean scores of Conscientiousness: No necessarily paradoxical findings. Journal of Research in Personality, 44, 630-640.

Nettle, D., \& Pollet, T. V. (2008). Natural selection on male wealth in humans. The American Naturalist, 172, 658-666.

Olderbak, S., Gladden, P., Wolf, P. S. A., \& Figueredo, A. J. (2014). Comparison of life history strategy measures. Personality and Individual Differences, 58, 82-88.

Pérusse, D. (1993). Cultural and reproductive success in industrial societies: Testing the relationship at the proximate and ultimate levels. Behavioral and Brain Sciences, 16, 267-283.

Ponzi, D., Flinn, M.V., Muehlenbein, M.P., Nepomnaschy, P.A. (2020). Hormones and human developmental plasticity, Molecular and Cellular Endocrinology, doi: https://doi.org/10.1016/j.mce.2020.110721.

Stewart-Williams, S., \& Thomas, A. G. (2013). The ape that thought it was a peacock: Does evolutionary psychology exaggerate human sex differences? Psychological Inquiry, 24, $137-168$.

Stulp, G., Sear, R., Schaffnit, S. B., Mills, M. C., \& Barrett, L. (2016). The reproductive ecology of industrial societies, part II: The association between wealth and fertility. Human Nature, 27, 445-470.

Tropf, F. C., Stulp, G., Barban, N., Visscher, P. M., Yang, J., Snieder, H., \& Mills, M. C. (2015b). Human fertility, molecular genetics, and natural selection in modern societies. PLOS ONE, 10, e0126821.

Udry, J. R., \& Cliquet, R. L. (1982). A cross-cultural examination of the relationship between ages at menarche, marriage, and first birth. Demography, 19, 53-63.

von Rueden, C. R., \& Jaeggi, A. V. (2016). Men's status and reproductive success in 33 nonindustrial societies: Effects of subsistence, marriage system, and reproductive strategy. Proceedings of the National Academy of Sciences USA, 113, 10824-10829.

Winegard, B., Winegard, B., \& Geary, D. C. (2018). The status competition model of cultural production. Evolutionary Psychological Science, 4, 351-371.

Winking, J., \& Koster, J. (2015). The Fitness effects of men's family investments. Human Nature, 26, 292-312.

Woodley of Menie, M. A., Cabeza de Baca, T., Fernandes, H. B. F., Madison, G., Figueredo, A. J., \& Peñaherrera Aguirre, M. (2017). Slow and steady wins the race: K positively predicts fertility in the USA and Sweden. Evolutionary Psychological Science, 3, 109 117. 


\section{S4. Reply to Zietsch \& Sidari (2020)}

This is a point-by-point reply to the extended critique of the fast-slow paradigm in humans by Zietsch \& Sidari (2020), henceforth Z\&S. Instead of repeating the arguments presented in the main text, I briefly summarize them and point to the relevant sections. The aim of this supplement is to organize the material in a convenient format, and clarify the points of agreement and disagreement with Z\&S.

\section{Z\&S, Section 2: The fast-slow continuum applied to inter-individual trait covariation within human populations}

Z\&S: The r-K (or fast-slow) continuum fell out of favor in biology [...] modern biology research that invokes the term 'life history theory' rarely adopts the fast-slow framework.

Reply: The fast-slow continuum is a robust empirical pattern (Section 2.1), and the term is routinely used to describe patterns of life history variation across species (for recent examples see Bakewell et al., 2020; Healy, 2019; Salguero-Gómez, 2017). The $r / K$ framework is a specific theoretical approach that has been used to explain the fast-slow continuum. While $r / K$ models have been out of favor for some time since the 1980s, they have experienced a resurgence in recent years (Section 2.2).

Z\&S: The empirical evidence for a unitary fast-slow continuum is mixed when looking across species within clades and controlling for body size.

Reply: This is a common misconception, largely based on the comparative studies by Bielby et al. (2007) and Jeschke \& Kokko (2009). In fact, much of the apparent instability of the fast-slow continuum is an artifact of axis rotation in PCA (Section 2.1; supplementary material $\mathrm{S} 1$ ). When the data of these papers are properly reanalyzed, the fast-slow continuum turns out to be robust to mass correction, and much less variable across species than often believed (see supplementary material S1 for details).

The word "unitary" might be taken to mean that, for the fast-slow continuum to be valid, the structure of life history strategies must be one-dimensional. But it has been clear from the beginning that there are other dimensions besides fast vs. slow, and that at least two axes are needed to describe broad patterns of variation in animals and plants (Section 2.1).

\section{Z\&S, Section 3: Species differ largely because of selection, whereas individuals differ largely because of inheritance}

Z\&S: Within populations as well, individuals differ in part because of genetic differences [...], but there is no equivalent (i.e. Darwinian) process that tailors varying individual genotypes to individuals' varying personal environments. [...] There is no equivalent evolutionary process creating inter-individual trait covariation. Selection and evolution can lead to phenotypic plasticity and adaptive calibration of individuals' traits to their personal environments [...]; but Darwinian phenotype-environment matching at the species level and plasticity at the individual level are completely different processes and may or may not lead to equivalent predictions regarding trait covariation. 
Reply: Z\&S are right to stress that individual differences are not like species/population differences, and are generated by different processes. Still, the two levels of organization may be functionally connected, so that individual variation within a population mirrors in important ways the patterns of variation between populations. I proposed the term "ecological gambit" for the working assumption that this is the case (Section 3.1). Of course, the assumption may or may not be warranted in any given case, and needs to be tested rather than simply taken for granted. In the context of life history strategies, basic trade-offs offer a possible functional link between population and individual differences (Section 3.1).

\section{Z\&S, Section 4: Exceptions to Mendel's law of independent assortment do not mean inter- individual trait covariation can be explained by Darwinian selection for trait clusters}

Z\&S: Intense correlational selection can in principle generate inter-individual trait covariation by generating transient covariation between genetic variants at different loci [...] However, even if such selection applied to human life history traits (for which there is no evidence), we show with empirical simulations (Supplementary Material) that the trait covariation it created would be weak and temporary, immediately eliminated once the correlational selection is relaxed. We further show that the greater the number of genetic variants underlying the traits, the weaker the temporary trait correlation.

Reply: Z\&S are right to point out that correlational selection per se is ephemeral, and linkage disequilibrium is not enough to produce robust patterns of covariation. However, persistent correlational selection may favor the evolution of pleiotropic effects that generate the same patterns, and pleiotropic effects can be robust and long-lasting (see Peiman \& Robinson, 2017; Roff \& Fairbairn, 2007). The same applies to fluctuating selection that acts simultaneously on multiple traits (Pavličev et al., 2011; Section 3.3).

Z\&S: Quantitative traits are underlain by variation at thousands of loci across the genome, so there is no reason to expect physical linkage to cause such traits to covary in adaptively helpful ways.

Reply: To my knowledge, physical linkage has not been proposed as an explanation of covariation among life history-related traits in humans.

Z\&S: Covariation between variants at different loci can be generated by non-random mating [...] However, the presence or absence of this covariation across loci does not pertain to whether the relevant traits "do or do not work together to serve their multiple adaptive functions" (Figueredo et al., 2013), and cross-trait assortative mating does not parallel evolutionary processes that create inter-species trait covariation.

Reply: This point is broadly correct. At the same time, it seems to imply that assortative mating only occurs for phenotypic condition/quality. But if there is also assortative mating for alternative life history strategies and/or life history-related traits (e.g., some aspects of personality), mating patterns can play a role in maintaining trait covariation.

Z\&S: Another way genetic variation in different traits can cluster is through pleiotropy, whereby the same genetic variant affects variation in multiple different traits. [...] Non-zero mutational effects tend to be both pleiotropic and deleterious [...] since they are random alterations to a complex, integrated design. This property of mutations would bias genetic correlations towards being directionally concordant with respect to overall quality or condition 
(creating a quality factor) [...]. Fig. 1, which shows genetic correlations among varied human traits relating to physical and mental robustness, is consistent with such a tendency.

Reply: The role of deleterious mutation is clearly important, and I agree that it has not received the attention it deserves in human research based on the fast-slow paradigm (Section 3.3). The genetic correlation matrix shown in Z\&S' Figure 1 contains measures of well-being and physical health, plus neuroticism, intelligence, physical traits such as stature and BMI, and three psychiatric disorders (depression, schizophrenia, and ADHD). The matrix makes the point that physical and mental conditions share common genetic sources that may be summarized by a general "quality" factor; but its relevance to life history strategies is unclear. While some authors have proposed a general factor of physical and mental health ("covitality") as a component of slow life histories (Figueredo et al., 2004, 2007), this is by no means a universal assumption in the field. In fact, the relations between mutation load, health, and life history allocations are likely more complex, as the same authors have acknowledged in other publications (Sefcek \& Figueredo, 2010). For a detailed account of these relations in the domain of mental health, see Del Giudice (2018).

Z\&S: Nor should we assume the same pattern across species, because: 1) the rationale for directional pleiotropy of mutational effects within species does not apply to inter-species trait covariation; 2) genetic variants causing inter-individual trait (co)variation need not be the same as those causing inter-species trait (co)variation; and 3) selection on different traits varies across species (which is largely why species themselves vary).

Reply: These points are all correct; however, the mechanisms that generate pleiotropic effects (e.g., hormones) can be highly conserved across species, and have the potential to link within- and between-species patterns of variation, particularly among closely related species (Section 3.1). Cross-level similarity cannot be simply assumed (which is why the ecological gambit is a gambit), but there may be reasons that make it more or less plausible in any given case (Section 3.1).

Z\&S: Directional pleiotropy as described above is consistent with a range of observed trait covariances in humans: for example, the positive manifold in diverse cognitive abilities [...]; the positive genetic correlation between most psychiatric disorders (including those that are hypothesised to be at opposite ends of the fast-slow spectrum, such as schizophrenia and autism; Del Giudice, 2014)

Reply: I agree with Z\&S that directional pleiotropy of the kind they describe contributes to the risk for many mental disorders, and to the pattern of positive correlations that gives rise to the so-called " $p$ factor" of psychopathology. At the same time, I have argued that the $p$ factor is not a unitary construct but a composite of three functionally distinct and largely separable sources of variation: (1) risk for "fast spectrum" disorders, that is, disorders associated with fast strategies and their phenotypic correlates; (2) risk for "defense activation" disorders (depression, generalized anxiety, panic, phobias, PTSD, and a subtype of OCD); and (3) low cognitive ability, which is strongly influenced by mutation load and, as shown by Z\&S in their Figure 1, correlates with health measures along a general "quality" dimension (Del Giudice, 2018). This alternative model accounts for the observed large-scale structure of psychopathology, and correctly predicts that factor analysis will recover a seemingly unitary p factor (see Del Giudice, 2016a, 2018). 
The example of autism vs. schizophrenia deserves a closer look. As noted by Z\&S, there is a small positive genetic correlation between autism and schizophrenia (e.g., Grove et al., 2019; Warrier et al., 2019). But while schizophrenia is negatively associated with IQ at the genetic and phenotypic level, polygenic scores for autism show a weak positive correlation with IQ (Clarke et al., 2016; Hagenaars et al., 2016; Grove et al., 2019). The genetic association with risk-taking is positive for schizophrenia but negative for autism (Linnér et al., 2018); there is also some evidence that polygenic scores for autism and schizophrenia tend to predict age at first intercourse and first birth in opposite directions - earlier for schizophrenia, later for autism (Ni et al., 2019; note that this study found a mix of significant and non-significant results using different methods, and indications of a U-shaped relation between schizophrenia polygenic scores and age at first birth). Of note, genetic risk for schizophrenia has been found to predict larger numbers of sexual partners (Lawn et al., 2019). Because of their overall correlation, both autism and schizophrenia load on a GWAS-based genetic $p$ factor; but if two components are extracted instead of just one, autism and schizophrenia end up loading on different components (Selzam et al., 2018). This pattern mirrors the phenotypic distribution of autistic-like and schizotypal traits, and is compatible with a diametrical model (see Del Giudice et al., 2014).

Taken together, these findings clearly indicate that directional pleiotropy is only part of the story. I have argued that a life history perspective can help make sense of the empirical literature, in view of the strong phenotypic and genetic heterogeneity of autism (Warrier et al., 2019). Specifically, I have suggested that "autism spectrum disorders" comprise two main functionally independent subtypes: a (mostly) high-functioning subtype, with a large contribution of common genetic variants and a specific cognitive/behavioral profile associated with slow strategies; and a (mostly) low-functioning subtype with a high risk of intellectual disability, a large contribution of rare/de novo mutations, and no apparent link with life history strategies (Del Giudice, 2018). This distinction is consistent with the genetic evidence on the role of mutations in autism (Gardner et al., 2019; Iossifov et al., 2014, 2015; Ronemus et al., 2014), and is supported by convergent epidemiological data on paternal/maternal age, socioeconomic status, and so forth (details in Del Giudice, 2018, Ch. 10).

If this hypothesis is correct, genetic correlations based on a unitary diagnosis of "autism spectrum disorder" reflect a mixture of functionally distinct conditions and symptom dimensions, and may hide as much as they reveal. For example, the shared role of deleterious alleles in the development of schizophrenia and (low-functioning) autism may mask the existence of negative relations between specific components/subtypes of the two disorders.

On a related note, the major impact of deleterious alleles on schizophrenia risk does not preclude a role for balancing selection (Keller, 2018), and is consistent with a life history/sexual selection account of this disorder (see Del Giudice, 2017, 2018). (Note that schizophrenia is also heterogeneous, and there are still some unanswered questions regarding its overlap with autism; see Del Giudice, 2018, Ch. 8.) This example illustrates that it is entirely possible to reconcile the idea of a fast-slow continuum with the existence of a pervasive dimension of genetic quality, and that the fast-slow distinction can be used with more nuance than implied by Z\&S.

Z\&S: Some have claimed that genetic correlation among human life history traits 'supports the hypothesis that Life History Strategy is predominantly under the control of regulatory genes that coordinate the expression of an entire array of life history traits' (Figueredo, Vásquez, Brumbach, \& Schneider, 2004). But genetic correlation does not imply any such thing. A genetic correlation between two traits could reflect one heritable trait directly influencing the other, or one heritable trait influencing environmental conditions that in turn influence the other trait, or both traits being influenced by a third heritable trait, or the traits being positively or negatively linked by shared developmental processes, among various other causal possibilities. 
Reply: This argument is technically correct, but ignores what we already know about the actual mechanisms of trait covariation in humans and other animals. For example, there is ample evidence that interlinked endocrine systems such as the HPA and HPG axes regulate key life history allocations (to survival, growth, reproduction, mating, parenting, and so forth) and coordinate the development and expression of multiple traits (Section 3.3). As Z\&S note, a genetic correlation does not imply a particular mechanism of covariation, but the idea that life history(-related) traits are coordinated by pleiotropic regulatory mechanisms is highly plausible. This does not imply the absence of the other causal pathways listed by Z\&S.

Z\&S: the genetic architecture of quantitative traits, which is absent any large-effect, pleiotropic 'genetic switches', is incompatible with the existence of regulatory genes that coordinate the expression of an entire array of life history traits (Penke, Denissen, \& Miller, 2007, p. 568).

Reply: Here, Z\&S seem to assume that regulatory genes can only act in isolation as large-effect "switches." But trait coordination is typically mediated by complex regulatory mechanisms (e.g., endocrine systems), whose functioning parameters are themselves highly polygenic (Section 3.3). In other words, there is no contradiction between the idea of pleiotropic regulatory genes and the empirically observed architecture of quantitative traits. Z\&S cite Penke et al. (2007), who based their own argument on the classic notion that balancing selection can only produce intermediate-frequency alleles of large effect. This idea has been overturned in the recent theoretical literature, as discussed in Section 3.3 and acknowledged by Penke and Jokela (2016) in their update of the 2007 paper (more details in the supplementary material S2).

\section{Z\&S, Section 5: Claims regarding correspondence between inter-species and inter- individual trait covariation are usually not based on cogent theory}

Z\&S: Those who have used observations or theory from inter-species trait covariation to explain (or predict) inter-individual trait covariation have usually not specified why they should correspond. [...] To our knowledge the life history literature in humans is absent any explicit description of a Darwinian process that should align human trait covariation with inter-species trait covariation. [...] A recent systematic review showed that the few pertinent formal models do not provide consistent or unique predictions regarding inter-individual covariation among life history and other traits (Mathot \& Frankenhuis, 2018), leaving the pace-of-life perspective without a clear theoretical basis.

Reply: Z\&S are right to point out that the link between the species and individual level of variation has been simply assumed, or justified with generic arguments (but see Wright et al., 2019). This is a major limitation of the fast-slow paradigm and a crucial topic for research, including formal modeling. In Section 3.1 I have tried to clarify the arguments in favor of crosslevel similarity, and explicitly noted that the assumption of similarity is in fact a theoretical "gambit" that may or may not succeed in any given case.

Z\&S: Wright, Bolstad, Araya-Ajoy, and Dingemanse (2019) proposed density-dependent fluctuating selection as a mechanism that might align inter-species and inter-individual trait covariation. [...] Its applicability to human trait covariation is doubtful though: modelling predicts that trait variation maintained by fluctuating selection will be explained disproportionately by alleles of intermediate frequency [...], whereas the genetic architecture of 
human quantitative traits that have been examined exhibits the opposite tendency, i.e. disproportionate contribution of rare alleles.

Reply: The model by Wright et al. (2019) is an interesting attempt to explicitly link between- and within-species variation in life history strategies. The particular explanation proposed by Wright et al. (fluctuating density-dependent selection) may or may not prove correct in the case of humans; however, Z\&S' argument against it rests on the outdated assumption that fluctuating selection (a specific kind of balancing selection) necessarily leads to an overwhelming contribution of common alleles to the genetic architecture of a trait. I discuss why this assumption is not supported by current theoretical models in Section 3.3 and the supplementary material S2.

\section{Z\&S, Section 6: Genetic coadaptation vs. adapted developmental plasticity}

Z\&S: A possibility is that scholars reading this might accept that genetic coadaptation does not viably align inter-species and inter-individual trait covariation, while still maintaining that these types of covariation are aligned by species-typical adaptations that tailor individuals' traits to the environments in which those individuals developed.

Reply: Given that genetic coadaptation is in fact quite plausible (see above), there is no need to discard it as an explanation. Moreover, the phenotypic effects of genetic and environmental variation may often align, owing to the fact that they are both channeled through the same coordination mechanisms (Section 3.3). This possibility is broadly consistent with the fact that phenotypic and genetic correlations between the same traits tend to have the same sign and a similar magnitude. (In biology, this correspondence is known as "Cheverud's conjecture;" see Section 3.3.)

Z\&S: A large proportion of variation in life history and related traits is attributable to genetic variation among individuals, and little is attributable to variation in the shared environment (i.e. the developmental home environment shared within twin pairs, including socioeconomic status, parenting style, father absence, risky upbringing). [...] The remainder of the variation in such traits tends to be mainly accounted for by residual factors, which include measurement error and random or idiosyncratic effects (biological or environmental) unshared by twin pairs. [...] Reported associations between developmental environment and adult traits are rarely controlled for genetic confounders [...] and when they are controlled the associations are often weaker or null. In light of these observations, a perspective focussed on adapted responses to early environmental conditions does not seem promising as a broad framework for explaining human trait covariation.

Reply: Z\&S make two important points about the small size of shared environmental effects in behavior genetic studies, and the lack of genetic control in most developmental research. These findings challenge to the role of developmental plasticity in the development of individual differences, including life history-related traits. In principle, there are ways to reconcile a degree of plasticity with the behavior genetic evidence (e.g., Del Giudice, 2015, 2016b; Section 3.3), but current ideas only scratch the surface and many crucial questions remain unanswered. We also urgently need to more information about the predictive value of early life factors, which in turn depends on the statistical properties of the environment (Section 3.3; Frankenhuis et al., 2019). 
Z\&S: Another problem with this perspective is that its most central hypothesis, that an adapted response to harsh environments (e.g. higher mortality and resource stress) should be to activate a faster life history strategy [...] is not justified by formal evolutionary modelling (Baldini, 2015). [...] For example, depending on how population density affects population fertility and how environmental harshness is defined (e.g. mortality rate, or effectiveness of investments in survival, growth, or reproduction), harsh environments are often predicted to lead to slower not faster life histories (Baldini, 2015). Further, the optimal strategies often comprise some 'slow' features and some 'fast' features (Baldini, 2015), contrary to the idea that trait covariation should cohere around a unitary fast-slow continuum.

Reply: I agree with Z\&S that there is a need for more formal models of life history strategies, especially at the within-population level. To be fair, the role of density-dependence has been addressed by some authors in this area (e.g., Ellis et al., 2009), even if it has not been emphasized in later research. On the other hand, it is important to acknowledge the limitations of Baldini's (2015) models. These models contain some implausible assumptions and a nonstandard definition of mortality. Baldini's key results critically depend on these questionable aspects of the models (see Section 3.1; André \& Rousset, 2020).

\section{Z\&S, Section 7: Empirical evidence does not support inter-individual trait covariation being organised around a fast-slow continuum in humans or in other species}

Z\&S: empirical support is weak for the hypothesis that inter-individual covariation of physiological, behavioural, and life history traits cohere around a fast-slow continuum (a hypothesis that derives from observations of inter-species trait covariation). Observed covariation of self-reported life history indicators in humans does not fit a model involving a single fast-slow dimension.

Reply: This is a crucial point that needs to be addressed on two levels. First and most important, the idea that individual differences are partly organized along a fast-slow continuum does not imply that the pattern of covariation among traits should be adequately described by a single factor. There are several reasons for this, including the existence of other functional axes of variation (which are also observed at the species level; see Section 2.1), the confounding effects of condition/quality, and the fact that many traits show nonlinear or interactive relations with life history outcomes (Section 3.4). Second, many commonly used inventories include putative indicators that have not been validated against life history variables and/or are not well suited as indicators (see Copping et al., 2017; Richardson et al., 2017).

Z\&S: More fundamentally, it is difficult to assess what human trait covariation is in line with a fast-slow continuum and what is not, because a trait's directionality as 'fast' or 'slow' is often inferred from the direction of its observed correlation with other supposed fast-slow traits or factors. For example, neuroticism is characterised by worry, self-doubt, and caution, and is accordingly associated with low-risk taking [...] But in exploratory factor analyses, neuroticism actually loads negatively on a 'K-factor' (i.e. tends to correlate positively with 'fast' traits); so high neuroticism is then hypothesised to indicate a fast life history strategy and its factor loading taken as supporting evidence (e.g. Figueredo et al., 2007; Richardson et al., 2017). This kind of circularity makes the theory nearly unfalsifiable and the existing evidence hard to evaluate.

Reply: I agree with the concern raised by Z\&S; circularity and lack of external validation are important limitations of certain approaches to the fast-slow continuum in humans. 
Z\&S: Evidence from a meta-analysis of empirical data in the animal literature is strikingly unsupportive of the hypothesis that inter-individual covariation of traits should cohere around a fast-slow continuum (Royauté, Berdal, Garrison, \& Dochtermann, 2018). The mean correlation among traits expected to positively covary was 0.06; within vertebrates it was 0.02. [...] Overall this meta-analysis seems to us a clear disconfirmation of the fast-slow continuum as a general organising principle for inter-individual trait covariation.

Reply: Z\&S are right to point out that the meta-analysis by Royauté et al. (2018) yielded largely negative findings. It is also important to understand what exactly has been disconfirmed, namely, the specific list of predictions advanced by Réale et al. (2010) about the behavioral and physiological correlates of fast vs. slow strategies. As I discuss in Section 3.2., those predictions were admittedly tentative, and the authors did not expect them to apply widely across species. Unfortunately, Réale et al.'s list has been reified and applied automatically, without testing its assumptions or adapting it to the ecology of different species (see also Del Giudice, 2018). This has been a major problem in the POLS literature, compounded by the questionable validity of standard behavioral measures (Royauté et al., 2018; see also Beckmann \& Biro, 2013; Carter et al., 2012). Hopefully, this meta-analysis will prompt researchers to improve their methodology and develop more sophisticated predictions.

\section{Z\&S, Section 8: Trade-offs}

Z\&S: most of the work on non-human animals that invokes the term 'life history theory' does not involve the concept of fast and slow strategies. Instead it tends to emphasise specific trade-offs in how individuals allocate limited energy/resources to different aspects of their life histories [...] In this approach it is not generally argued that covariation between different life history traits should be understood per Darwinian principles, such that traits correlate because they 'work well together'; rather the trait correlations are thought to result from specific tradeoffs that are due to fundamental limitations of an individual's resources such as energy or time.

Reply: Contrary to what Z\&S seem to imply here, the fact that fundamental constraints on energy and time drive life history trade-offs is not an alternative to the notion that traits covary because they "work well together;" in fact, constraints are what shapes the logic of adaptive trait coordination. Trade-offs force organisms to make "decisions" within constraints; the implementation of those decisions requires the coordination of behavior, physiology, and morphology in ways that "work well together," in the sense that they tend to bring about adaptive outcomes without wasting precious resources (Section 3.2). To avoid confusion, it may be useful to distinguish between classic life history traits such as longevity and fertility — which are the outcomes of the organism's allocations - and the life history-related traits (behavioral, physiological, morphological) that mediate those allocations (Section 3.2).

Z\&S: It is problematic, though, to assume that trade-offs within individuals should cause corresponding trait covariation across individuals [...] individuals differ in the amount of bioenergetic resources they have or can acquire (e.g. due to variation in mutation load or favourability of their environment), and so the covariation between traits that functionally tradeoff within an individual can covary positively across individuals, depending on the means and variances in resource acquisition vs. allocation of those resources.

Reply: This is an important point that has been noted repeatedly in the theoretical literature (e.g., Rezinck et al., 2000; Roff \& Fairbairn, 2007; Wilson, 2014), but not consistently applied to life history variation in humans and other animals. Clearly, the existence of individual 
differences in condition/resources can mask the functional structure of trade-offs. However, this is not an insurmountable problem as much as an opportunity for progress: in many cases, it should be possible to control for the effects of individual condition and recover a clearer picture of the underlying trade-offs (Section 3.4; for an empirical example see McLean et al., 2019).

Z\&S: Further, Houle (1991) showed, under the assumption that genetic variation in fitness-relevant traits is maintained by mutation-selection balance, that inter-individual genetic covariation of fitness-relevant traits depends on the underlying functional architecture of the loci that affect the traits-in particular, the relative number of loci involved in acquiring versus allocating resources. That is, 'studies estimating $G$ [genetic correlations among traits] do not test for the existence of life-history tradeoffs' (Houle, 1991, p. 630).

Reply: Z\&S are correct, and their point is a reminder that genetic correlations cannot be simply taken at face value as reflections of the underlying functional relations (Section 3.4). However, the situation is not fundamentally different at the genetic vs. phenotypic level: in both cases, the effects of individual condition/quality can be accounted for (at least in principle) so as to reveal the underlying trade-offs (e.g., McLean et al., 2019; Wilson, 2014).

Overall summary: Z\&S are right to point out the difference between the level of population/species differences and that of individual variation. However, there can be functional links between the two levels (e.g., basic trade-offs) that justify the ecological gambit as a working assumption. Another important contribution of $Z \& S$ is to highlight the role of mutations and broad "quality" factors. But while those factors may potentially mask the functional structure of trade-offs at the phenotypic and genetic level, they can be accounted for within a more comprehensive model. Moreover, genetic quality alone is clearly insufficient to explain the totality of the evidence, for example regarding the functional basis of autism vs. schizophrenia. I argue that $Z \& S$ are wrong to discount the importance of phenotypic integration and dismiss the role of balancing selection and pleiotropic regulatory mechanisms. However, they raise important questions about the evidence for adaptive plasticity, the empirical support for trait covariation, and the methodological and conceptual problems of popular measurement approaches.

\section{References}

André, J. B., \& Rousset, F. (2020). Does extrinsic mortality accelerate the pace of life? A barebones approach. Evolution and Human Behavior.

Baldini, R. (2015). Harsh environments and" fast" human life histories: What does the theory say? BioRxiv, 014647.

Bakewell, A. T., Davis, K. E., Freckleton, R. P., Isaac, N. J., \& Mayhew, P. J. (2020). Comparing life histories across taxonomic groups in multiple dimensions: How mammallike are insects? The American Naturalist, 195, 70-81.

Beckmann, C., \& Biro, P. A. (2013). On the validity of a single (boldness) assay in personality research. Ethology, 119, 937-947.

Bielby, J., Mace, G. M., Bininda-Emonds, O. R., Cardillo, M., Gittleman, J. L., Jones, K. E., ... \& Purvis, A. (2007). The fast-slow continuum in mammalian life history: An empirical reevaluation. The American Naturalist, 169, 748-757. 
Carter, A. J., Marshall, H. H., Heinsohn, R., \& Cowlishaw, G. (2012). How not to measure boldness: novel object and antipredator responses are not the same in wild baboons. Animal Behaviour, 84, 603-609.

Clarke, T. K., Lupton, M. K., Fernandez-Pujals, A. M., Starr, J., Davies, G., Cox, S., et al. (2016). Common polygenic risk for autism spectrum disorder (ASD) is associated with cognitive ability in the general population. Molecular Psychiatry, 21, 419-425.

Copping, L. T., Campbell, A., Muncer, S., \& Richardson, G. B. (2017). The psychometric evaluation of human life histories: A reply to Figueredo, Cabeza de Baca, Black, Garcia, Fernandes, Wolf, and Woodley (2015). Evolutionary Psychology, 1474704916663727.

Del Giudice, M. (2015). Plasticity as a developing trait: Exploring the implications. Frontiers in Zoology, 12, S4.

Del Giudice, M. (2016a). The life history model of psychopathology explains the structure of psychiatric disorders and the emergence of the p factor: A simulation study. Clinical Psychological Science, 4, 299-311.

Del Giudice, M. (2016b). Differential susceptibility to the environment: Are developmental models compatible with the evidence from twin studies? Developmental Psychology, 52, 1330-1339.

Del Giudice, M. (2017). Mating, sexual selection, and the evolution of schizophrenia. World Psychiatry, 16, 141-142.

Del Giudice, M. (2018). Evolutionary psychopathology: A unified approach. New York: Oxford University Press.

Del Giudice, M., Klimczuk, A. C. E., Traficonte, D. M., \& Maestripieri, D. (2014). Autistic-like and schizotypal traits in a life history perspective: Diametrical associations with impulsivity, sensation seeking, and sociosexual behavior. Evolution \& Human Behavior, $35,415-424$.

Ellis, B. J., Figueredo, A. J., Brumbach, B. H., \& Schlomer, G. L. (2009). The impact of harsh versus unpredictable environments on the evolution and development of life history strategies. Human Nature, 20, 204-268.

Figueredo, A. J., Cabeza de Baca, T., \& Woodley, M. A. (2013). The measurement of human life history strategy. Personality and Individual Differences, 55, 251-255.

Figueredo, A. J., Vásquez, G., Brumbach, B. H., \& Schneider, S. M. R. (2004). The heritability of life history strategy: The k-factor, covitality, and personality. Social Biology, 51, 121143.

Figueredo, A. J., Vasquez, G., Brumbach, B. H., \& Schneider, S. M. R. (2007). The K-factor, covitality, and personality: A psychometric test of life history theory. Human Nature, 18 , 47-73.

Frankenhuis, W. E., Nettle, D., \& Dall, S. R. (2019). A case for environmental statistics of earlylife effects. Philosophical Transactions of the Royal Society of London B, 374, 20180110.

Grove, J., Ripke, S., Als, T. D., Mattheisen, M., Walters, R. K., Won, H., ... \& Awashti, S. (2019). Identification of common genetic risk variants for autism spectrum disorder. Nature genetics, 51, 431-444.

Hagenaars, S. P., Harris, S. E., Davies, G., Hill, W. D., Liewald, D. C., Ritchie, S. J., et al. (2016). Shared genetic aetiology between cognitive functions and physical and mental health in UK Biobank ( $=112$ 151) and 24 GWAS consortia. Molecular Psychiatry, 21, $1624-1632$. 
Healy, K., Ezard, T. H., Jones, O. R., Salguero-Gómez, R., \& Buckley, Y. M. (2019). Animal life history is shaped by the pace of life and the distribution of age-specific mortality and reproduction. Nature Ecology \& Evolution, doi: 10.1038/s41559-019-0938-7

Houle, D. (1991). Genetic covariance of fitness correlates: What genetic correlations are made of and why it matters. Evolution, 45, 630-648.

Iossifov, I., Levy, D., Allen, J., Ye, K., Ronemus, M., Lee, et al. (2015). Low load for disruptive mutations in autism genes and their biased transmission. Proceedings of the National Academy of Sciences, 112, E5600-E5607.

Iossifov, I., O’Roak, B. J., Sanders, S. J., Ronemus, M., Krumm, N., Levy, D., et al. (2014). The contribution of de novo coding mutations to autism spectrum disorder. Nature, 515, 216221.

Jeschke, J. M., \& Kokko, H. (2009). The roles of body size and phylogeny in fast and slow life histories. Evolutionary Ecology, 23, 867-878.

Keller, M. C. (2018). Evolutionary perspectives on genetic and environmental risk factors for psychiatric disorders. Annual Review of Clinical Psychology, 14, 471-493.

Mathot, K. J., \& Frankenhuis, W. E. (2018). Models of pace-of-life syndromes (POLS): A systematic review. Behavioral Ecology and Sociobiology, 72, 41.

Lawn, R. B., Sallis, H. M., Taylor, A. E., Wootton, R. E., Smith, G. D., Davies, N. M., ... \& Munafo, M. R. (2019). Schizophrenia risk and reproductive success: A Mendelian randomization study. Royal Society Open Science, 6, 181049.

Linnér, R. K., Biroli, P., Kong, E., Meddens, S. F. W., Wedow, R., Fontana, M. A., ... \& Okbay, A. (2018). Genome-wide study identifies 611 loci associated with risk tolerance and risky behaviors. BioRxiv, 261081.

McLean, E. M., Archie, E. A., \& Alberts, S. C. (2019). Lifetime fitness in wild female baboons: Trade-offs and individual heterogeneity in quality. The American Naturalist, 194, 745759.

Ni, G., Amare, A. T., Zhou, X., Mills, N., Gratten, J., \& Lee, S. H. (2019). The genetic relationship between female reproductive traits and six psychiatric disorders. Scientific Reports, 9, 12041.

Pavličev, M., Cheverud, J. M., \& Wagner, G. P. (2011). Evolution of adaptive phenotypic variation patterns by direct selection for evolvability. Proceedings of the Royal Society B, 278, 1903-1912.

Peiman, K. S., \& Robinson, B. W. (2017). Comparative analyses of phenotypic trait covariation within and among populations. The American Naturalist, 190, 451-468.

Penke, L., Denissen, J. J., \& Miller, G. F. (2007). The evolutionary genetics of personality. European Journal of Personality, 21, 549-587.

Penke, L., \& Jokela, M. (2016). The evolutionary genetics of personality revisited. Current Opinion in Psychology, 7, 104-109.

Reznick, D., Nunney, L., \& Tessier, A. (2000). Big houses, big cars, superfleas and the costs of reproduction. Trends in Ecology and Evolution, 15, 421-425.

Richardson, G. B., Sanning, B. K., Lai, M. H. C., Copping, L. T., Hardesty, P. H., \& Kruger, D. J. (2017). On the psychometric study of human life history strategies: State of the science and evidence of two independent dimensions. Evolutionary Psychology, 1474704916666840.

Roff, D. A., \& Fairbairn, D. J. (2007). The evolution of trade-offs: Where are we? Journal of Evolutionary Biology, 20, 433-447. 
Ronemus, M., Iossifov, I., Levy, D., \& Wigler, M. (2014). The role of de novo mutations in the genetics of autism spectrum disorders. Nature Reviews Genetics, 15, 133-141.

Royauté, R., Berdal, M. A., Garrison, C. R., \& Dochtermann, N. A. (2018). Paceless life? A meta-analysis of the pace-of-life syndrome hypothesis. Behavioral Ecology and Sociobiology, 72, 64.

Salguero-Gómez, R. (2017). Applications of the fast-slow continuum and reproductive strategy framework of plant life histories. New Phytologist, 213, 1618-1624.

Sefcek, J. A., \& Figueredo, A. J. (2010). A life-history model of human fitness indicators. Biodemography and Social Biology, 56, 42-66.

Selzam, S., Coleman, J. R., Caspi, A., Moffitt, T. E., \& Plomin, R. (2018). A polygenic p factor for major psychiatric disorders. Translational psychiatry, 8, 205.

Warrier, V., Toro, R., Won, H., Leblond, C. S., Cliquet, F., Delorme, R., ... \& Grove, J. (2019). Social and non-social autism symptoms and trait domains are genetically dissociable. Communications Biology, 2, 328.

Wright, J., Bolstad, G. H., Araya-Ajoy, Y. G., \& Dingemanse, N. J. (2019). Life-history evolution under fluctuating density-dependent selection and the adaptive alignment of pace-of-life syndromes. Biological Reviews, 94, 230-247.

Zietsch, B. P., \& Sidari, M. J. (2020). A critique of life history approaches to human trait covariation. Evolution and Human Behavior, doi: 10.1016/j.evolhumbehav.2019.05.007 